\title{
Mid-Infrared-Selected Quasars. I. Virial Black Hole Mass and Eddington Ratios
}

\section{Citation}

Dai, Y. Sophia, Martin Elvis, Jacqueline Bergeron, Giovanni G. Fazio, Jia-Sheng Huang, Belinda J. Wilkes, Christopher N. A. Willmer, Alain Omont, and Casey Papovich. 2014. "Mid-InfraredSelected Quasars. I. Virial Black Hole Mass and Eddington Ratios." The Astrophysical Journal 791 (2) (August 5): 113. doi:10.1088/0004-637x/791/2/113.

\section{Published Version}

doi:10.1088/0004-637X/791/2/113

\section{Permanent link}

http://nrs.harvard.edu/urn-3:HUL.InstRepos:30212132

\section{Terms of Use}

This article was downloaded from Harvard University's DASH repository, and is made available under the terms and conditions applicable to Other Posted Material, as set forth at http:// nrs.harvard.edu/urn-3:HUL.InstRepos:dash.current.terms-of-use\#LAA

\section{Share Your Story}

The Harvard community has made this article openly available.

Please share how this access benefits you. Submit a story.

\section{Accessibility}




\title{
MID-INFRARED-SELECTED QUASARS. I. VIRIAL BLACK HOLE MASS AND EDDINGTON RATIOS*
}

\author{
Y. Sophia Dai (戴昱 $)^{1,2}$, Martin Elvis ${ }^{1}$, Jacqueline Bergeron ${ }^{3}$, Giovanni G. Fazio ${ }^{1}$, Jia-Sheng Huang ${ }^{1}$, Belinda \\ J. Wilkes ${ }^{1}$, Christopher N. A. Willmer ${ }^{4}$, Alain OMOnT ${ }^{3}$, And CASEy Papovich ${ }^{5}$ \\ ${ }^{1}$ Harvard-Smithsonian Center for Astrophysics, 60 Garden Street, Cambridge, MA 02138, USA \\ ${ }^{2}$ Caltech/IPAC, 1200 East California Boulevard, Pasadena, CA 91125, USA; ydai@ @altech.edu \\ ${ }^{3}$ CNRS, UMR7095, Institut d'Astrophysique de Paris, F-75014 Paris, France \\ ${ }^{4}$ Steward Observatory, University of Arizona, 933 North Cherry Avenue, Tucson, AZ 85721, USA \\ ${ }^{5}$ Department of Physics and Astronomy, Texas A\&M University, College Station, TX 77843, USA \\ Received 2013 November 5; accepted 2014 June 12; published 2014 August 5
}

\begin{abstract}
We provide a catalog of 391 mid-infrared-selected (MIR; $24 \mu \mathrm{m}$ ) broad-emission-line (BEL; type 1) quasars in the $22 \mathrm{deg}^{2}$ SWIRE Lockman Hole field. This quasar sample is selected in the MIR from Spitzer MIPS with $S_{24}>400 \mu \mathrm{Jy}$, jointly with an optical magnitude limit of $r(\mathrm{AB})<22.5$ for broad line identification. The catalog is based on MMT and Sloan Digital Sky Survey (SDSS) spectroscopy to select BEL quasars, extending the SDSS coverage to fainter magnitudes and lower redshifts, and recovers a more complete quasar population. The MIR-selected quasar sample peaks at $z \sim 1.4$ and recovers a significant and constant (20\%) fraction of extended objects with SDSS photometry across magnitudes, which were not included in the SDSS quasar survey dominated by point sources. This sample also recovers a significant population of $z<3$ quasars at $i>19.1$. We then investigate the continuum luminosity and line profiles of these MIR quasars, and estimate their virial black hole masses and the Eddington ratios. The supermassive black hole mass shows evidence of downsizing, although the Eddington ratios remain constant at $1<z<4$. Compared to point sources in the same redshift range, extended sources at $z<1$ show systematically lower Eddington ratios. The catalog and spectra are publicly available online.
\end{abstract}

Key words: catalogs - galaxies: active - galaxies: high-redshift - galaxies: Seyfert - infrared: galaxies - quasars: general - quasars: supermassive black holes

Online-only material: color figures, supplemental data

\section{INTRODUCTION}

The apparent connection between supermassive black holes (SMBHs) and their host galaxies has been explained by a variety of theories. In the merger-driven model, the collision of dust-rich galaxies drives gas inflows, fueling both starbursts and buried quasars until feedback disperses the gas and dust, allowing the quasar to be briefly visible as a bright optical source (e.g., Sanders et al. 1988; Hopkins et al. 2006). Instead of physical coupling between the black hole $(\mathrm{BH})$ and host galaxy, the central-limit theorem can be used to explain the linear SMBH mass and bulge mass correlation by the hierarchical assembly of BH and stellar mass (Peng 2007; Jahnke \& Macciò 2011). Alternatively, the cold flow model (e.g., Dekel et al. 2009; Bournaud et al. 2011; Di Matteo et al. 2012) introduces inflowing cosmological cold gas streams, rather than collisions, to fuel the star formation and quasar, and better explains the clumpy disks observed for high- $z$ galaxies. Observationally, a SMBH-host connection is supported by the discovery of the correlations of the SMBH mass with bulge luminosity, mass, and velocity dispersion, especially with bulges and ellipticals (e.g., Kormendy \& Richstone 1995; Ferrarese \& Merritt 2000; McLure \& Dunlop 2002; Kormendy \& Ho 2013). However, despite tremendous progress on the demographic studies of SMBHs, whether or how the SMBH regulates the formation and evolution of its hosts via the possible "feedback" process is still under debate. One sign of such feedback may be the ongoing star formation observed for host galaxies of active galactic nuclei (AGNs) and quasars, and vice versa—starbursts are found to

\footnotetext{
* Observations reported here were obtained at the MMT Observatory, a joint facility of the Smithsonian Institution and the University of Arizona.
}

host buried AGNs (Kauffmann et al. 2003; Shi et al. 2009; Dai et al. 2012). Based on the similar star formation rate observed for galaxies with and without an AGN, recent studies suggest that the SMBH-host correlation results from the gas availability, instead of major interaction between the SMBH growth and host star formation (e.g., Goulding et al. 2014; Lilly et al. 2013). In this paper, we present a mid-infrared (MIR) selection to effectively select quasar candidates with dusty nuclear material in a disk/wind or torus geometry (e.g., Elvis 2000; Antonucci 1993; Urry \& Padovani 1995, "torus" hereafter). This selection is relatively unaffected by obscuration.

In the high redshift $(z>0.5)$ universe, it is difficult to observe broad-line (type 1) quasars and their host galaxies simultaneously. The quasar glare usually outshines the host galaxy at optical wavelengths, and the host has a small angular size. In large optical surveys, the focus has been on broademission-line (BEL) quasars (e.g., Richards et al. 2006b; Shen et al. 2011, S11), or "blue" quasars, which are biased toward optically unobscured (Type 1) objects with limited information about the host galaxy. Studies on the cosmic history of quasars show an evolution over redshifts, with a quasar peak appearing at $z \sim 1.5$ (e.g., Hasinger et al. 2005; Silverman et al. 2008). At longer infrared (IR) wavelengths, where thermal emission from dust is dominant, quasars have characteristic power-law-shaped MIR spectral energy distributions (SEDs), and are selected by different color wedges in the Spitzer Infrared Array Camera (Fazio et al. 2004) and Wide-field Infrared Survey Explorer (Wright et al. 2010) bands (Lacy et al. 2004; Sajina et al. 2005; Stern et al. 2005, 2012; Donley et al. 2012).

Recent surveys in the IR have detected optically obscured (type 2), dust-reddened quasars (e.g., Richards et al. 2003, 2009; Polletta et al. 2006; Glikman et al. 2012; Lacy et al. 2013). 
These quasars are marked by having reddened UV-optical SEDs resulting from dust absorption. At different redshift and luminosity ranges, quasars are reported to have an obscuration fraction from $20 \%$ to more than $50 \%$ (Lacy et al. 2002; Glikman et al. 2004, 2007; Urrutia et al. 2009; Juneau et al. 2013; Lacy et al. 2013). In the merger-driven model these quasars are in an early transitional phase and are in the process of expelling their dusty environment before becoming "normal" blue quasars (type 1). This IR-luminous phase also evolves with time, and was more common at high $z$ (e.g., Caputi et al. 2007; Serjeant et al. 2010). Optical studies of quasar and host systems are challenged by the high contrast between the bright-point-source quasar and starlight. Infrared-selected quasars are good candidates to study the SMBH-host connection, as they are not biased against dusty hosts.

In this paper, we present a catalog of 391 MIR-selected BEL objects in the $\sim 22 \mathrm{deg}^{2}$ Lockman Hole-Spitzer Wide-area InfraRed Extragalactic Survey (LHS) Field (SWIRE; Lonsdale et al. 2003). As will be pointed out in Section 2.6, because all the objects have BEL features and the majority also qualify the classical Seyfert/quasar luminosity separation $\left(M_{\mathrm{B}}<-23\right)$, we will hereafter simply refer to these BEL objects as quasars. Combining the MIR $24 \mu \mathrm{m}$ flux limit and optical identification has been demonstrated to be an effective way of selecting quasars (with a 13\% detection rate in Papovich et al. 2006). This MIR selection was designed to be biased toward dusty systems, where ample hot dust exists in the nuclear region with a higher likelihood of tracing remnant or ongoing star formation (cool dust). The spectroscopic sample used in this work comprises new observations taken with the Hectospec at the MMT of the wide-angle SWIRE field and a smaller MIPS GTO field, and spectra obtained by the Sloan Digital Sky Survey (SDSS) within the Lockman Hole footprint. We hope that this sample will provide a new test bed to study the SMBH self-regulation or AGN feedback when the system has not relaxed to equilibrium, if such effects do exist. In Section 2, we review the sample selection and introduce the spectroscopic data and the MIR additions to the SDSS quasar catalog, followed by the spectral measurements in Section 3. In Sections 4 and 5, we describe the virial $\mathrm{BH}$ mass and bolometric luminosity estimates; we then follow with the spectral catalog (Section 6), a discussion (Section 7), and summary (Section 8). Throughout the paper, we assume a concordance cosmology with $H_{0}=70 \mathrm{~km} \mathrm{~s}^{-1} \mathrm{Mpc}^{-1}$, $\Omega_{\mathrm{M}}=0.3$, and $\Omega_{\Lambda}=0.7$. All magnitudes are in $\mathrm{AB}$ system except where otherwise noted.

\section{THE SAMPLE}

\subsection{MIR MIPS 24 um Selection}

The combined MIR $24 \mu \mathrm{m}$ and optical selection for this survey was designed to detect objects with luminous torus/ nucleus and not biased against dusty hosts. The MIR selection allows for the detection of hot dust (a few hundred Kelvin) at the redshifts $z \sim 1.5$; while optical follow-up spectroscopically identified the BEL objects, confirming their unobscured (type 1) quasar nature. This MIR selection also allows for a far-infrared (FIR) cross-match to look for cool dust for SMBH-host studies, as demonstrated in Dai et al. (2012).

We select Spitzer MIPS (Rieke et al. 2004) $24 \mu \mathrm{m}$ sources from the SWIRE survey in the $\sim 22 \mathrm{deg}^{2}$ LHS field centered at R.A. = 10:46:48, decl. = 57:54:00 (Lonsdale et al. 2003). The SDSS imaging also covers the LHS region to $r=22.2$ at $95 \%$ detection repeatability, but can go as deep as $r=23$. All magnitudes are taken from the SDSS photoObj catalog in DR7, which are already corrected for Galactic extinction according to Schlegel et al. (1998). They are the SDSS approximate AB system (Oke \& Gunn 1983; Fukugita et al. 1996; Smith et al. 2002). SDSS has astrometric uncertainties $<0$ '. 1 on average. ${ }^{6}$ In Figure 1 we show the SWIRE and SDSS coverages in the LHS field.

We first apply a $24 \mu \mathrm{m}$ flux limit of $400 \mu \mathrm{Jy}(\sim 8 \sigma)$, which yields a sample of 23, 402 objects. The completeness at $400 \mu \mathrm{Jy}$ for the SWIRE-MIPS catalog is $~ 90 \%$ (Shupe et al. 2008). The confusion limit due to extragalactic sources for the MIPS $24 \mu \mathrm{m}$ band is $56 \mu \mathrm{Jy}$ (Dole et al. 2004), so source confusion is not an issue in this sample. The errors in position for these sources are between $0^{\prime \prime} .2$ and $0^{\prime \prime} .4$, and the effective beam size (FWHM) of MIPS at $24 \mu \mathrm{m}$ is $\sim 6^{\prime \prime} .7$

We then match the $24 \mu \mathrm{m}$ flux-limited sources to the SDSS DR7 catalog. We determine an association radius of $2^{\prime \prime} .5$ to maximize the matching number counts while at the same time minimizing the cases of random association (Figure 2). We first match the SWIRE and the SDSS $r$-band catalogs. Then we offset the SWIRE position by a random number within $10^{\prime \prime}$ radius, and match them to the SDSS $r$-band catalog. The association radius is determined by comparing the random association rate at different radii. The random association rate within $5^{\prime \prime}$ is $\sim 18 \%$ (in 2467 out of 14,069 matches), but decreases to $\sim 6 \%$ within 2 .'5. Beyond 2 ..5 radius there are $>50 \%$ random associations. The estimated total number of false associations within 2.5 is $868(6 \%)$. We find 14,069 matches when adopting the association radius of 2.5 . Of these, $87 \%(12,255)$ of the $24 \mu \mathrm{m}$ sources also satisfy $r<22.5$. This $r$ limit allows for follow-up optical spectroscopic observations with the MMT.

The optical spectroscopic survey consists of four parts (Figure 3, Table 1). The first three subsamples-(1) SDSS DR7, (2) MMT 2009 survey (MMT09), and (3) MMT 2005 bright targets (MMT05b) - include the MIR-selected targets as described above. A fourth subsample comes from MMT 2005 observations for MIPS deep targets (Section 2.4): (4) MMT 2005 faint targets (MMT05f) $\left(60 \mu \mathrm{Jy} \leqslant S_{24}<400 \mu \mathrm{Jy}\right)$, and is kept only for comparison purpose. Table 2 summarizes the MMT covered observations.

\subsection{SDSS Spectroscopy}

In order to minimize the need for new spectroscopy, we downloaded and analyzed the existing SDSS spectroscopy of LHS MIPS $24 \mu \mathrm{m}$ targets directly from the SDSS DR7 SkyServer. ${ }^{8}$ The SDSS spectra have a resolving power of $R \sim$ 1800-2200, with a wavelength coverage of 3800-9200 A. In this study, we use the "1D" calibrated spectra from the DR7 Data Archive Server, ${ }^{9}$ which is stored in a logarithmic pixel scale of $10^{-4}$. We made a SQL search (with a $5^{\circ}$ radius, $r<22.5$ ) in the SDSS DR7 SpecObj catalog ${ }^{10}$ and found 2978 objects. The redshifts given in the SDSS DR7 SpecObj catalog were determined by the spectro1d package (Stoughton et al. 2002). Spectra for all SDSS objects with redshifts in the LHS field were downloaded, irrespective of their SDSS classification. We matched these sources with the SWIRE MIPS $24 \mu \mathrm{m}$ catalog, and excluded 2019 SDSS targets that were not detected by

\footnotetext{
6 http://www.sdss.org/dr7

7 http://irsa.ipac.caltech.edu/data/SPITZER/docs/mips/ mipsinstrumenthandbook/

8 http://cas.sdss.org/dr7

9 http://das.sdss.org/spectro

10 http://cas.sdss.org/astrodr7/en/tools/search/sql.asp
} 


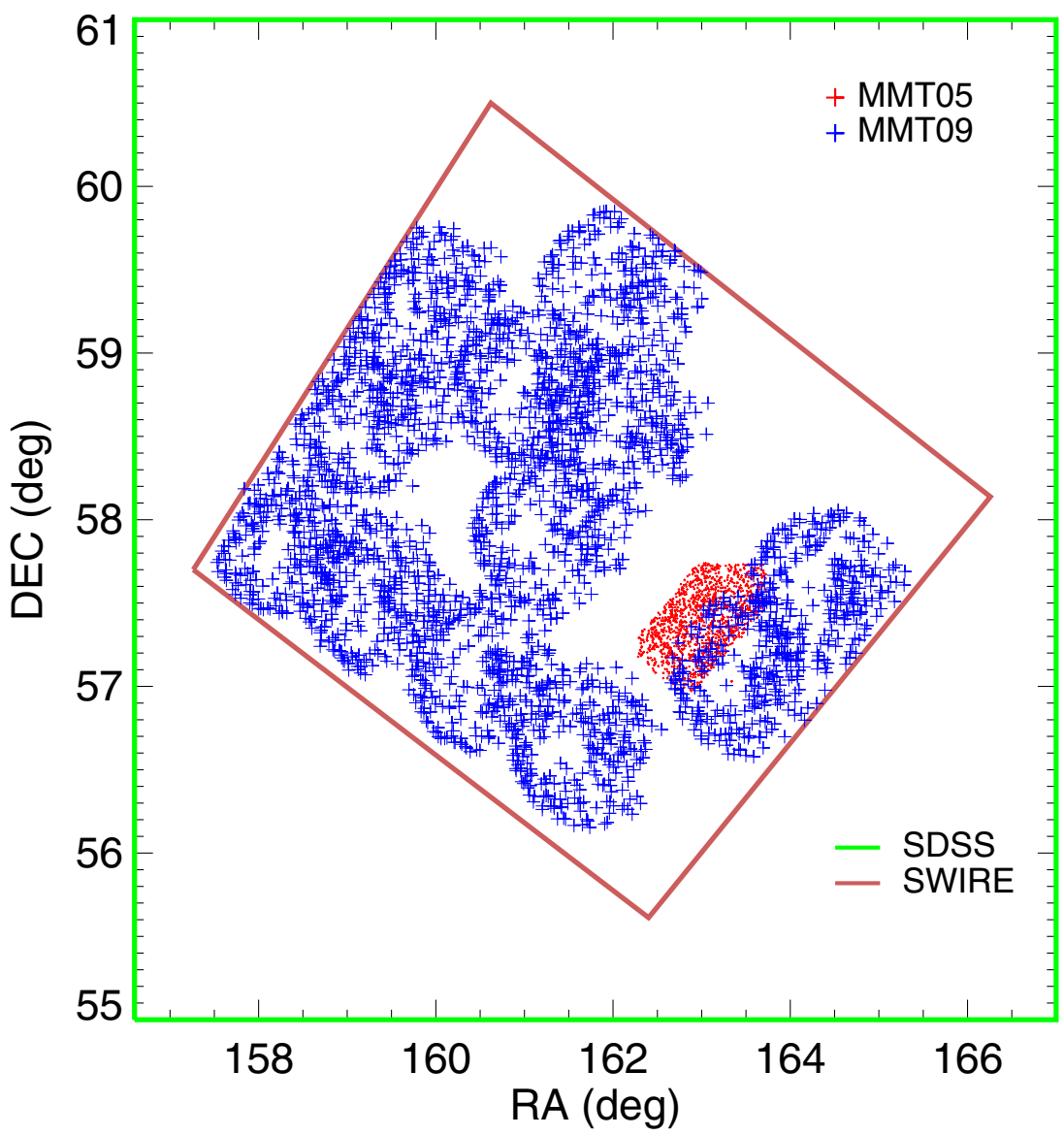

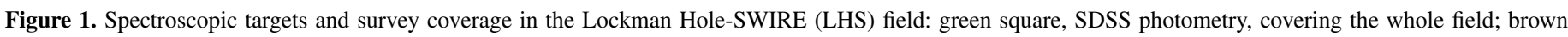

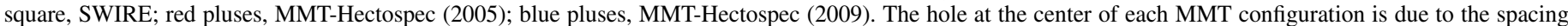
limitation of the 300 fibers in the Hectospec instrument.

(A color version of this figure is available in the online journal.)

SWIRE, and 38 SDSS targets with $S_{24}<400 \mu \mathrm{Jy}$. Within the remaining 921 qualified spectra, we only retained, for BEL identification, the 854 objects $(93 \%)$ with a redshift confidence $\geqslant 0.9$.

\subsection{MMT 2009 Spectroscopy}

Hectospec is a 300 fiber spectrometer with a $1^{\circ}$ diameter field of view (FOV) mounted on the MMT (Fabricant et al. 2005; Mink et al. 2007). The combination of a wide field with a large aperture makes Hectospec well suited to cover extended areas, such as the LHS. Hectospec covers a wavelength range of 3650-9200 $\AA$ with a $6 \AA$ resolution $\left(1.2 \AA\right.$ pixel $^{-1}$, $R=600-1500)$. The primary spectroscopic data specific to this study were taken in 2009 (MMT09, PI: Huang), over 11 dark photometric nights with good seeing $\left(<2^{\prime \prime}\right)$ in 12 FOVs. The MMT data cover a total area of $\sim 12 \mathrm{deg}^{2}$ (50\% of LHS field). An ongoing MMT project (PI: Dai) is complementing the 2009 observations by targeting unobserved areas within the LHS. However, the new project adopts a different selection, which emphasizes Herschel (Pilbratt et al. 2010) targets to favor objects with cool dust $(<60 \mathrm{~K})$ that traces the host star formation. These data will be published in a forthcoming paper (Y. S. Dai et al., in preparation). In Figure 1 the spectroscopic targets in the 12 fields observed in 2009 are marked with blue pluses. At the center of each MMT FOV, an area with fewer targets can be noticed. This is due to the spacing limitations of Hectospec, whose 300 fibers cannot be crossed or placed less than $50^{\prime \prime}$ from one another. The $\sim 3000$ spectroscopic targets were selected from the 11, 401 MIPS and $r$-band flux-limited catalog from Section 2.1 (after excluding the 854 SDSS objects from Section 2.2). Brighter $24 \mu \mathrm{m}$ sources were given higher priority (See Figure 3), and fibers were configured to cover as much of the LHS field as possible. Hectospec gives a clear BEL detection (median signal-to-noise ratio (S/N) per pixel $>$ 5) for a $r=22.4$ quasar in a $1.5 \mathrm{hr}$ exposure (e.g., Figure 4, LHS-2009.0226-239). Thus $1.5 \mathrm{hr}$ exposures were used as the standard. Spectra for 2913 objects were recorded in 2009. The optical spectroscopic completeness in the 12 MMT09 Fields is $33 \%$ for $S_{24}>400 \mu$ Jy objects, with an average overlap of $0.08 \mathrm{deg}^{2}$ between different configurations. After taking into account the objects missing due to fiber placement limitations, the completeness of MMT09 sample drops to $\sim 30 \%$, and will be used in the following discussions (Section 2.7.)

\subsection{MMT 2005 MIPS-deep Spectroscopy}

This spectroscopic sample is extended to include 273 MMT spectra from an earlier 2005 deep survey (MMT05) across eight highly overlapping FOVs in the LHS. The 2005 data cover a much smaller $\left(\sim 0.5 \mathrm{deg}^{2}\right)$ region (PI: Papovich). The MMT 2005 survey applied a deeper $24 \mu \mathrm{m}$ flux limit of $S_{24}>60 \mu \mathrm{Jy}$, which is near to the MIPS confusion limit (Rieke et al. 2004). Only $r<22$ targets were selected and MMT05 recorded 1481 spectra. Of these, 273 objects also satisfy the bright MMT09 limit $\left(S_{24}>400 \mu \mathrm{Jy}\right)$ and are included in the primary sample. 


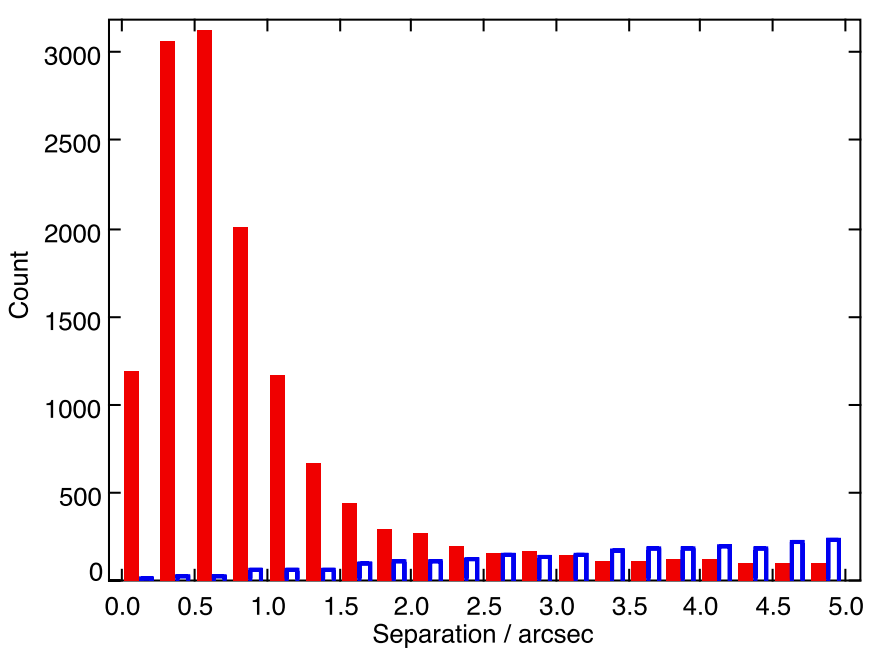

Figure 2. Histogram of the offset between SWIRE and SDSS positions (red). In blue is the same histogram for mismatches after a random position offset $\left(<10^{\prime \prime}\right.$ radius). The random association rate within $5^{\prime \prime}$ is $\sim 18 \%$, but declines to $\sim 6 \%$ within $2^{\prime \prime}$.5. A matching radius of $2^{\prime \prime} .5$ was used to maximize the matching counts while minimizing the random associations.

(A color version of this figure is available in the online journal.)

We call this the MMT05b (bright) sample. The remaining 1208 objects with fainter flux $\left(60<S_{24}<400 \mu \mathrm{Jy}\right)$ are also kept for comparison purposes. This sample is designated MMT05f (faint).

The highly overlapped MMT05 FOVs lead to an optical spectroscopy completeness of $66 \%$ for $24 \mu \mathrm{m}$ bright targets $\left(S_{24}>400 \mu \mathrm{Jy}\right)$ in the $0.5 \mathrm{deg}^{2}$ area. This higher completeness comes at the cost of lower efficiency, with an average overlap of $0.94 \mathrm{deg}^{2}$, and a drop from 242 targets per FOV in MMT09 observations to 185 targets per FOV in MMT05, which encouraged the adoption of the MMT09 strategy.

\subsection{Spectral Data Reduction}

The SDSS spectra and redshifts are used directly from the DR7 SpecObj catalog without further reduction. The MMT Hectospec data (MMT09, MMT05b, and MMT05f) were reduced using the HSRED pipeline (Cool et al. 2008, $\mathrm{http}: / / \mathrm{mmto}$.org/ rcool/hsred/index.html), which is based on the SDSS pipeline. HSRED extracts one-dimensional (1D) spectra, subtracts the sky, and then flux calibrates the spectra. The flux calibration is done using the spectra of 6-10 stars selected to have the SDSS colors of the F stars that are observed simultaneously with the main galaxy and quasar sample. The flux calibration correction is obtained combining the extinction-corrected SDSS photometry of these stars with Kurucz (1993) model fits (Cool et al. 2008). These stellar spectra are also used to remove the telluric lines. The spectral range covered by Hectospec allows for the detection of one or more typical emission lines present in the spectra of quasars and galaxies ( $\mathrm{C}$ IV, $\mathrm{Mg}$ II, $\mathrm{H} \beta$, [O III], $\mathrm{H} \alpha$ ), for galaxies to $z \sim 1$ and quasars to $z \sim 4.5$. The redshifts measured by HSRED also use a code adapted from SDSS and the same templates as SDSS. All spectra were visually inspected for validation as described below.

A redshift quality flag is assigned to each spectrum, following the same procedure used for the DEEP2 survey (Willmer et al. 2006; Newman et al. 2013), where redshift qualities range from $Q=4$ (probability $P>95 \%$ of being correct), 3 (90\% $<P<95 \%), 2(P<90 \%)$, and 1 (no features recognized).

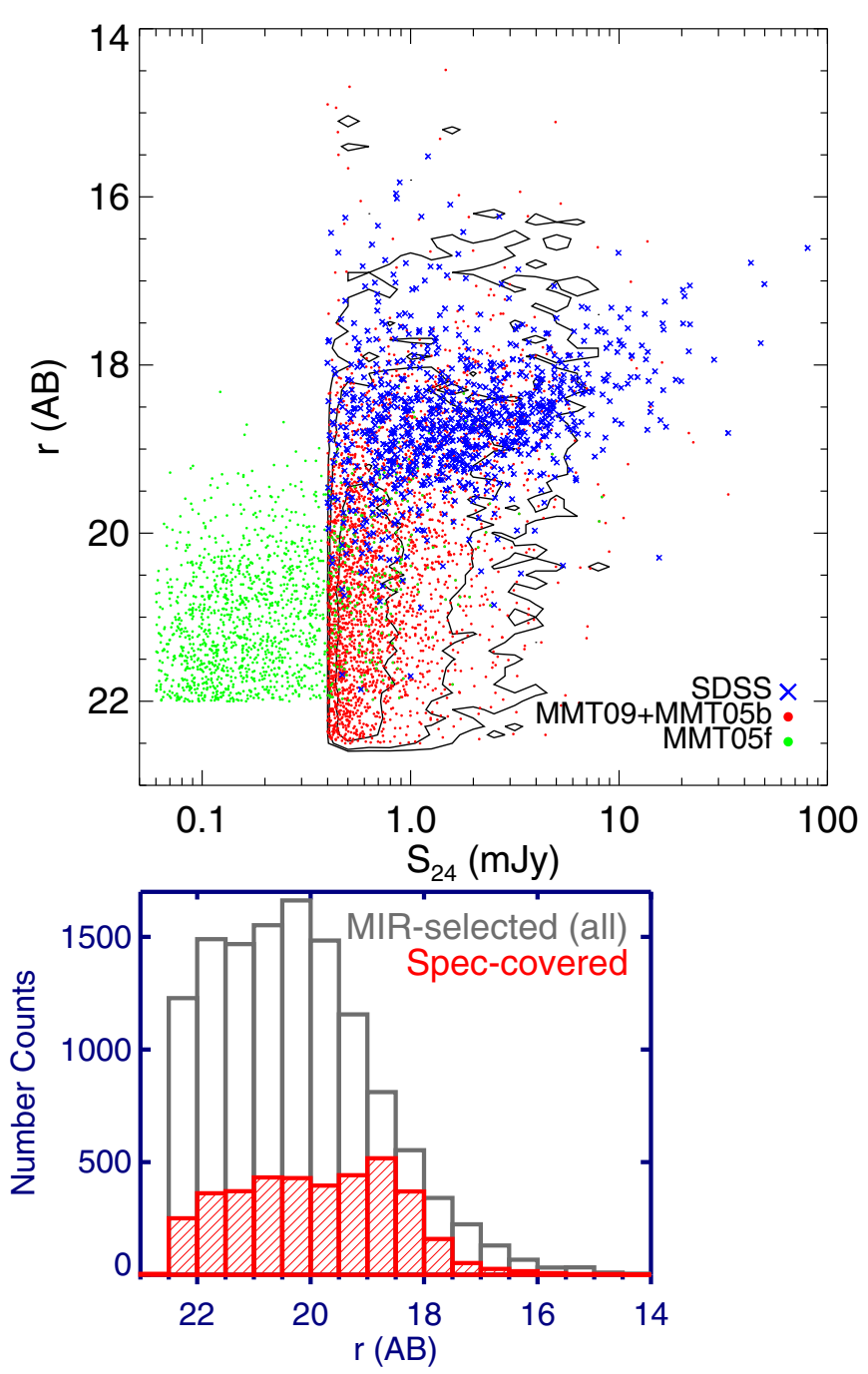

Figure 3. Top: spectroscopic coverage in the $S_{24}-r$ band space for MIR-selected targets: blue crosses, 854 SDSS DR7 targets; red, 2485 MMT09 plus 273 MMT05b targets; green, 902 MMT05f targets. The contours in the background are the 12,255 MIR-selected targets that satisfy the $S_{24}>400 \mu \mathrm{Jy}$ (and $r<$ 22.5) limits. Bottom: the $r$-band magnitude and $24 \mu \mathrm{m}$ flux distribution for all MIR-selected targets (black) and the spectroscopic covered objects (Speccovered, red).

(A color version of this figure is available in the online journal.)

$Q=2$ spectra are assigned to objects for which only a single feature is detected, but cannot be identified without ambiguity. The $Q=3$ spectra have more than one spectral feature identified, but tend to have low $\mathrm{S} / \mathrm{N}$. The typical confidence levels for these objects is $\sim 90 \%$ for the DEEP2 galaxies. Finally, $Q=4$ objects have two or more spectral features with reasonable to high $\mathrm{S} / \mathrm{N}$. The confidence level of these redshifts is typically $>95 \%$. Because of the larger spectral range covered by HECTOSPEC (3800-9500 ̊) relative to DEEP2 (5000-9500 $)$, we expect that the quoted confidence levels are the conservative limits for our spectra.

Figure 4 shows examples of objects in each redshift quality category. In this study, as for the 854 SDSS spectra, only spectra of $Q=3$ and 4 were used. This yields a total of 2485 MMT09 spectra ( $\sim 90 \%$ of all the recorded spectra) and 1175 MMT05 spectra $(\sim 80 \%)$. The entire 273 MMT05b subsample satisfies the redshift quality filter.

To summarize, we have a total of 3612 spectra of MIRselected objects with $r<22.5$ observed by MMT-Hectospec 
Table 1

Optical Spectroscopic Sample Summary

\begin{tabular}{lclcrrrr}
\hline \hline Source & $r_{\mathrm{AB}}$ & $\begin{array}{c}S_{24} \\
(\mu \mathrm{Jy})\end{array}$ & $N_{\text {spec }}$ & $N_{\text {quasar }}$ & $\begin{array}{r}\text { Covered } \\
\left(\mathrm{deg}^{2}\right)\end{array}$ & Detection Rate \\
\hline$(1)$ & SDSS & $<22.5$ & $>400$ & $854^{\mathrm{a}}$ & $138^{\mathrm{a}}$ & 22 & $16.2 \%$ \\
$(2)$ & MMT09 & $<22.5$ & $>400$ & 2485 & 226 & 11 & $9.1 \%$ \\
$(3)$ & MMT05b & $<22$ & $>400$ & 273 & 27 & 0.5 & $9.9 \%$ \\
\hline & Total & $<22.5$ & $>400$ & 3612 & 391 & 22 & $10.8 \%$ \\
\hline$(4)$ & MMT05f & $<22$ & $60<S_{24}<400$ & 902 & 17 & 0.5 & $1.9 \%$ \\
\hline
\end{tabular}

Note. ${ }^{\text {a }}$ In the full $22 \mathrm{deg}^{2}$ LHS field. The numbers of spectra and quasars in the $11.5 \mathrm{deg}^{2}$ MMT-covered regions are 622 and 96 , respectively.

Table 2

Observation Log for MMT Spectroscopic Survey

\begin{tabular}{|c|c|c|c|c|c|}
\hline Instrument & Telescope & $\begin{array}{c}\text { R.A. } \\
(\mathrm{J} 2000)\end{array}$ & $\begin{array}{c}\text { Decl. } \\
(\mathrm{J} 2000)\end{array}$ & $\begin{array}{c}\text { Exposure } \\
\text { (hr) }\end{array}$ & Observation Date \\
\hline Hectospec & MMT observatory & $+10: 39: 48.3$ & $+59: 16: 56$ & 1.5 & 2009.0319 \\
\hline Hectospec & MMT observatory & $+10: 48: 48.9$ & $+58: 31: 58$ & 1.5 & 2009.0318 \\
\hline Hectospec & MMT observatory & $+10: 33: 26.2$ & $+57: 55: 05$ & 1.5 & 2009.0317 \\
\hline Hectospec & MMT observatory & $+10: 45: 21.6$ & $+57: 53: 55$ & 1.5 & 2009.0301 \\
\hline Hectospec & MMT observatory & $+10: 37: 35.1$ & $+57: 32: 53$ & 1.2 & 2009.0228 \\
\hline Hectospec & MMT observatory & $+10: 39: 48.3$ & $+59: 16: 56$ & 1.5 & 2009.0227 \\
\hline Hectospec & MMT observatory & $+10: 42: 20.4$ & $+57: 05: 15$ & 1.5 & 2009.0226 \\
\hline Hectospec & MMT observatory & $+10: 37: 12.5$ & $+58: 38: 24$ & 1.5 & 2009.0223 \\
\hline Hectospec & MMT observatory & $+10: 54: 13.1$ & $+57: 03: 57$ & 1.5 & 2009.0222 \\
\hline Hectospec & MMT observatory & $+10: 57: 45.5$ & $+57: 34: 04$ & 1.5 & 2009.0222 \\
\hline Hectospec & MMT observatory & $+10: 44: 31.2$ & $+58: 46: 14$ & 1.5 & 2009.0220 \\
\hline Hectospec & MMT observatory & $+10: 48: 29.3$ & $+59: 22: 27$ & 1.5 & 2009.0131 \\
\hline Hectospec & MMT observatory & $+10: 52: 31.3$ & $+57: 24: 15$ & 1.3 & 2005.0410 \\
\hline Hectospec & MMT observatory & $+10: 51: 02.9$ & $+57: 22: 17$ & 0.6 & 2005.0409 \\
\hline Hectospec & MMT observatory & $+10: 51: 46.1$ & $+57: 26: 32$ & 0.3 & 2005.0408 \\
\hline Hectospec & MMT observatory & $+10: 51: 02.9$ & $+57: 22: 17$ & 1.7 & 2005.0405 \\
\hline Hectospec & MMT observatory & $+10: 52: 09.7$ & $+57: 27: 49$ & 1.0 & 2005.0310 \\
\hline Hectospec & MMT observatory & $+10: 51: 42.2$ & $+57: 28: 01$ & 1.0 & 2005.0308 \\
\hline Hectospec & MMT observatory & $+10: 51: 42.2$ & $+57: 28: 01$ & 1.0 & 2005.0304 \\
\hline Hectospec & MMT observatory & $+10: 52: 03.8$ & $+57: 26: 22$ & 1.0 & 2005.0308 \\
\hline Hectospec & MMT observatory & $+10: 52: 18.8$ & $+57: 21: 53$ & 1.0 & 2005.0308 \\
\hline
\end{tabular}

or chosen from the SDSS SpecObj catalog with a redshift confidence of $>90 \%$ (Table 1 ).

\subsection{Broad-line Object Identification}

The 3612 reduced 1D SDSS and Hectospec spectra were fitted using our IDL program, which was adopted from the S11 procedure. This program fits a polynomial continuum $\left(S_{\text {continuum }}=A_{\lambda} \times\left(\lambda / \lambda_{0}\right)^{\alpha_{\lambda}}\right)$ and a Gaussian around the redshifted $\mathrm{C}$ IV, $\mathrm{Mg}$ II, and $\mathrm{H} \beta$ regions, based on the HSRED or SpecObj redshifts (see also Section 3). Objects are kept as quasar candidates if they have at least one BEL (FWHM > $1000 \mathrm{~km} \mathrm{~s}^{-1}$; Schneider et al. 2007) in the secure spectral ranges with limited atmospheric extinction and instrument errors: 3850-8400 $\AA$ (Fabricant et al. 2008) for MMT targets and 3850-9000 A (Stoughton et al. 2002) for SDSS targets. Outside these ranges, the spectra start to be bounded by sky-subtraction errors and are therefore not reliable. The MMT range is from Fabricant et al. (2008) - it was chosen to be most consistent $(<5 \%)$ with SDSS after comparing the optical spectra of the same targets. The IDL program identified 236 MMT09, 28 MMT05b, and 132 SDSS BEL objects, all of which have an emission line equivalent width (EW) greater than 6. Given our flux limit $(r<22.5)$, the majority of the BEL quasars $(83 \%$ with $\left.M_{i}<-23\right)$ also satisfy $M_{\mathrm{B}}<-23$, which is the quasar definition in Schmidt \& Green (1983; Figure 5). Since the SDSS quasar definition is also based on the BEL features (Schneider et al. 2007), we would refer to these BEL objects as quasars in the following text.

As a check, we visually examined all 3612 spectra from both the MMT and SDSS surveys. This process removed 22 MMT09, 1 MMT05b, and 5 SDSS objects that were erroneously identified as quasars due to bad fits. This process also added 12 MMT09 and 11 SDSS objects, but no MMT05b objects were missed due to a poor fit by the IDL program. Of the 11 SDSS objects, 6 were not included in the SDSS DR7 quasar catalog. The six new objects are confirmed as quasars with a broad $\mathrm{H} \beta$ emission line (Figure 6 shows one example). We will explore the possible reasons why they were missed in the SDSS DR7 catalog in Section 2.7.2. Special objects with interesting features — such as broad absorption line (BAL) and narrow absorption line (NAL) quasars - are also flagged (see Section 7).

Combining the IDL fit and eye check, we identified 226 quasars from the MMT09, 27 from the MMT05b, and 138 from the SDSS DR7 SpecObj catalogs. This adds up to a total of 391 MIR-selected quasars in the LHS field. For comparison, we also scanned the 902 fainter $\left(S_{24 \mu \mathrm{m}}<400 \mu \mathrm{Jy}\right)$ objects from MMT05f survey, and identified 17 BEL objects (one was added by eye). Table 1 summarizes the quasar numbers 


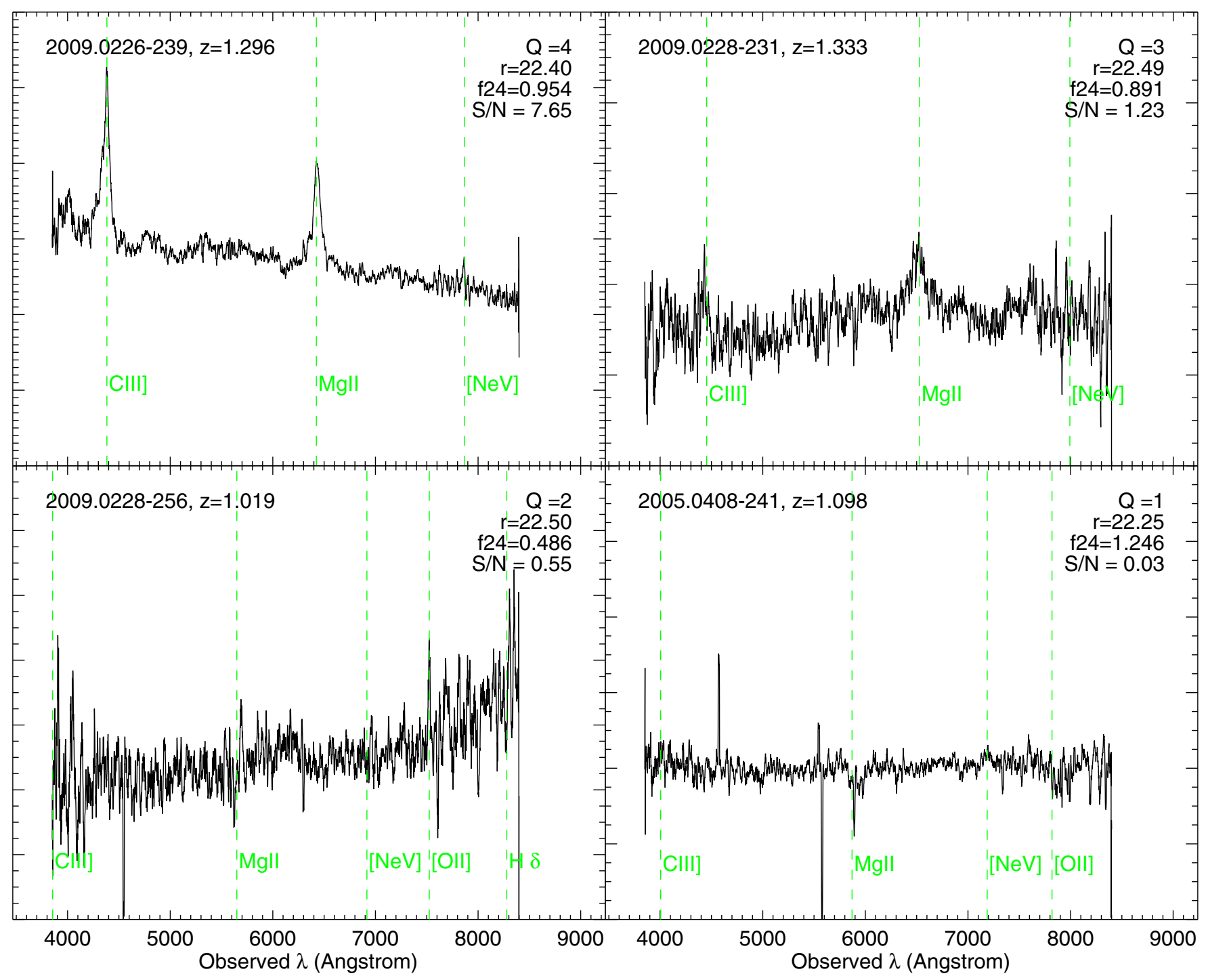

Figure 4. Examples of spectra with different redshift quality flags from $Q=4$ (probability $>95 \%$ of being correct) to $Q=1$ (no features recognized), following the same procedure as in the DEEP2 survey (Willmer et al. 2006; Newman et al. 2013). $Q$ values are listed in the top right corner; also shown in the top right corner are the SDSS $r$-band magnitude (AB), the MIPS $24 \mu \mathrm{m}$ flux in mJy, and the median S/N per pixel in the plotted region. (Note that this is different from the median S/N of the emission line region, which is usually of a higher value.) Typical quasar lines are marked in green. The redshifts given for the $Q=1$ and $Q=2$ examples are generated from the code or after visual check, and are not reliable values. Only spectra with a $z$ flag of $Q \geqslant 3$ are kept in this work (see also Section 2.5).

(A color version of this figure is available in the online journal.)

in each subsample. The fraction of MIR quasars is $9.1 \%$ in the MMT09 subsample, and $9.9 \%$ in the MMT05b subsample, which yields an average detection rate of $9.2 \%$. After including the SDSS quasars selected through color-color selection, the total detection rate for the MIR quasar sample in LHS field is $10.8 \%$. When only considering the MMT and SDSS overlapping area, the quasar detection rate is an almost identical $10.9 \%$. These detection rates are marginally lower than the $13 \% \pm 3 \%$ reported in Papovich et al. (2006), where a higher $24 \mu \mathrm{m}$ flux limit $\left(S_{24 \mu \mathrm{m}}>1 \mathrm{mJy}\right)$ was applied.

To study the overall properties of the MIR-selected quasars, we plot the redshift ( $z$, top), $r$-band magnitude ( $r$, middle), and $24 \mu \mathrm{m}$ flux ([$\left.S_{24}\right]$, bottom) distributions in Figure 7 . The sample has a redshift range of $0.07<z<3.93$, with a median redshift of 1.3. A K-S test shows a significant difference $(p \ll 0.1)$ between the SDSS and MMT subsamples in all three parameters $\left(z, S_{24}\right.$, and $\left.r\right)$. The SDSS quasars have two $z$ peaks at $1<z<2$ and at $z \sim 3.2$, with an overall median $z=1.5$. The double peaks are caused by the two main color selection criteria (ugri and griz) applied in SDSS for low- $z$ $(z<2.9)$ and high- $z(z>2.9)$ quasars (Section 2.7.1). The MMT, on the other hand, has a roughly Gaussian redshift distribution, with a peak at $z \sim 1.3$. The MIR-selected quasars are clearly not homogeneously distributed across redshifts. The SDSS subsample has an overall brighter $r$ and $S_{24}$ than the MMT subsample, and overlaps significantly with the bright end of the MMT quasars. These differences are due to the SDSS quasar algorithm, which has a limit at $i=20.2$, about two magnitudes brighter than the MMT selection $\left(r=22.5^{11}\right)$. The MMT-Hectospec survey intentionally dropped SDSS targets with existing spectra, leaving the MMT targets biased toward the faint end. The combination of the MMT and SDSS provides a better way to examine the completeness of MIR-selected quasars at $S_{24} \geqslant 400 \mu \mathrm{Jy}$.

\footnotetext{
11 Using Richards et al. (2006a) mean SDSS quasar template, $r=22.5$ is equivalent to $i=22.4$ at $z \sim 1.5$.
} 


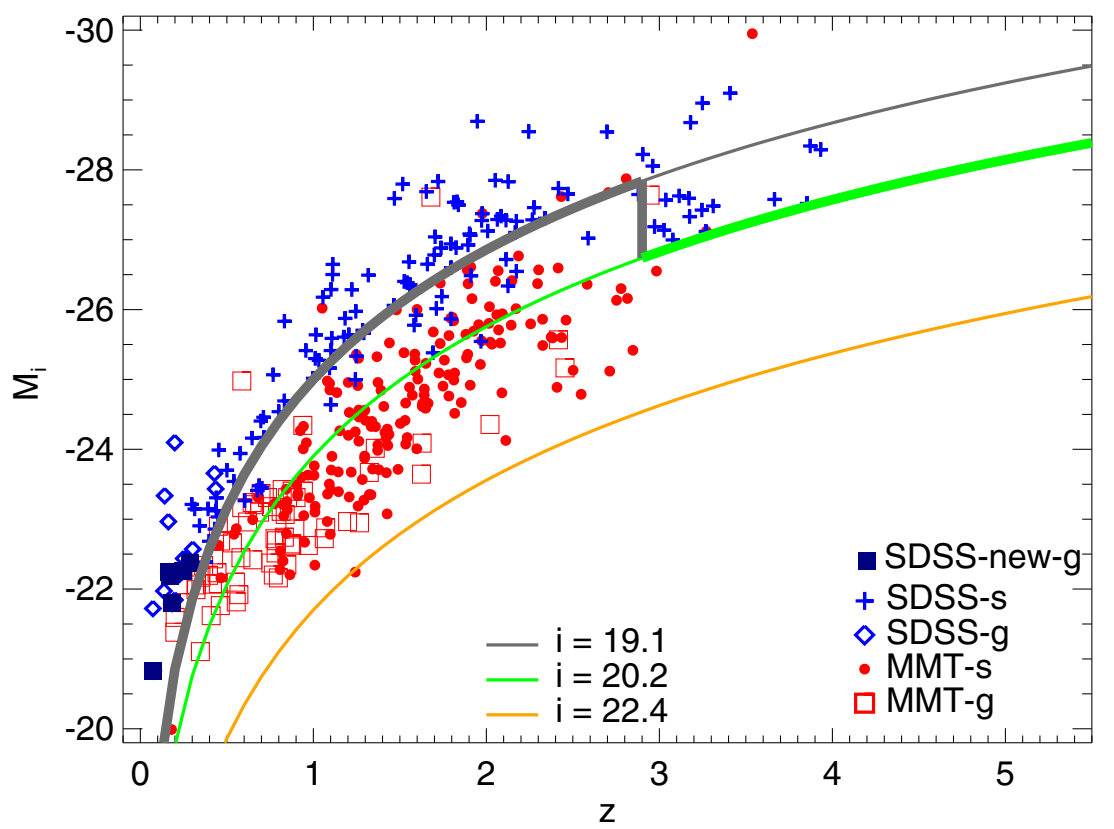

Figure 5. Distribution of MIR-selected quasars in the luminosity-redshift plane. Luminosity is indicated by $i$-band absolute magnitude, $M_{i}$, calculated from the SDSS photometric magnitude. In blue are the SDSS-identified quasars, with blue pluses for point sources and blue diamonds for extended sources; in red are the MMT-identified quasars, with red dots for point sources and red squares for extended sources. Whether an object is extended (galaxy-like, "g") or a point source (star-like, "s") is defined by the extendedness of the SDSS photometry; see Section 2.7.1. The solid navy blue squares mark the six quasars newly identified with SDSS spectra that were not included the SDSS DR7 quasar catalog. The curves show the two magnitude ranges of SDSS selections at $i=19.1$ (gray) and $i=20.2$ (green), and thick curves are the limiting $z$-dependent magnitudes SDSS used for the sample of spectroscopic targets (See Section 2.7.1). The orange curve shows the equivalent $i$-band magnitude of the MMT-Hectospec limit $(r=22.4)$. A total of 93 new quasars have been identified by MMT (red dots and squares) at $i<20.2$, of which 80 fall between $19.1<i<20.2$ at $z<3$, a region SDSS did not cover in the uniform color selection (See also Section 2.7). The number densities of $z<3$ quasars is $\sim 10 \mathrm{deg}^{-2}$ at $i<19.1$, which is slightly higher than the $\sim 9 \mathrm{deg}^{-2}$ at $19.1<i<20.2$.

(A color version of this figure is available in the online journal.)

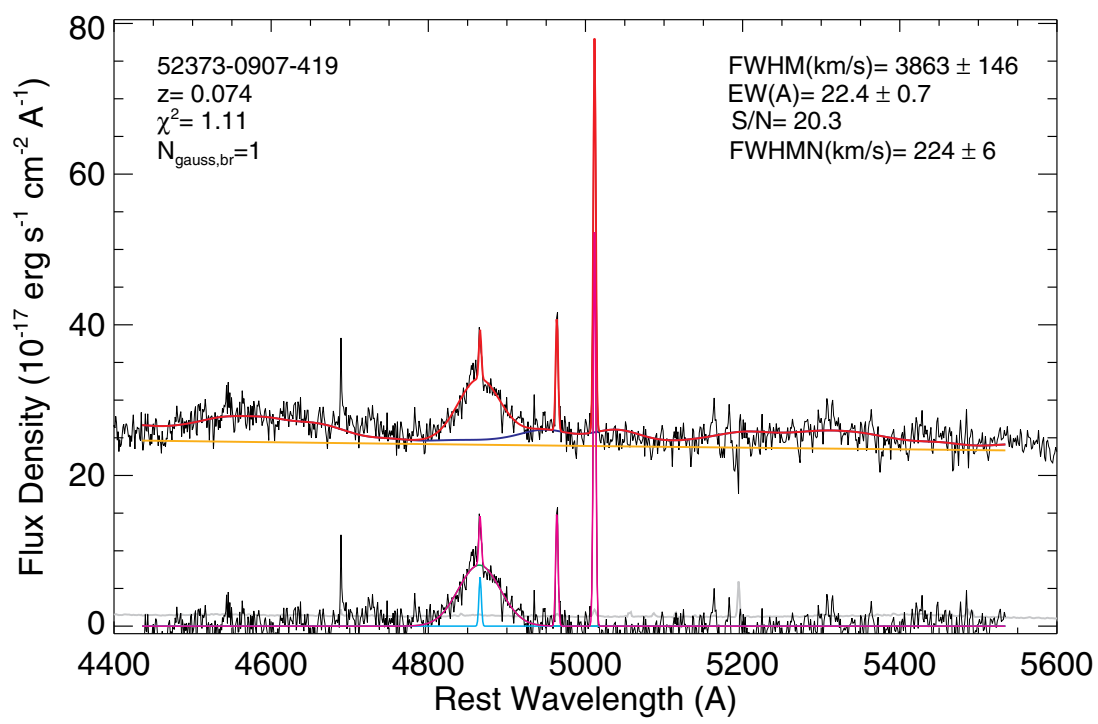

Figure 6. One example of the six newly identified SDSS quasars zoomed in at the H $\beta$ emission line region ([4400, 5600] $\AA$ ). The two upper sets of plots show the original spectrum (black), the estimated continuum (orange), the scaled Fe template (dark blue), and the final fitted composite spectrum (red). The lower plot shows the continuum- and Fe-template-subtracted spectrum (black), in addition to the variance spectrum (gray), the narrow-line emission component (cyan), and the wide-line component (green). The latter is mostly subsumed by the composite narrow+wide emission line spectrum shown in magenta. The top left corner shows the ID, redshift, fitting $\chi^{2}$, and number of Gaussian component used, and top right shows the fitting results (FWHM, EW, signal-to-noise, and the FWHM of the narrow line) in the emission line region.

(A color version of this figure is available in the online journal.)

In Figure 8 we compare the optical to MIR colors against the $r$ magnitude for the MMT and SDSS subsamples. The MIRselected MMT subsamples are redder in $r-\left[S_{24}\right]$ colors than the SDSS subsample, with median $\left[r-\left[S_{24}\right]\right]$ values of 4.0 for MMT09, 3.9 for MMT05b, and 3.3 for SDSS. Although separable by $S_{24}$ flux, the 17 MMT05f quasars $\left(60<S_{24}<400 \mu \mathrm{Jy}\right)$ show similar $r-\left[S_{24}\right]$ colors to the SDSS subsample, but are bluer (median $r-\left[S_{24}\right]=3.4$ ) than the MMT subsamples. A K-S test gives a probability of 0.975 of the MMT05f and SDSS objects, indicating identical distributions. Instead, the K-S test probability is $<0.001$ between MMT05 $\mathrm{f}$ and the brighter MMT subsamples (MMT09, MMT05b), indicating a significant 

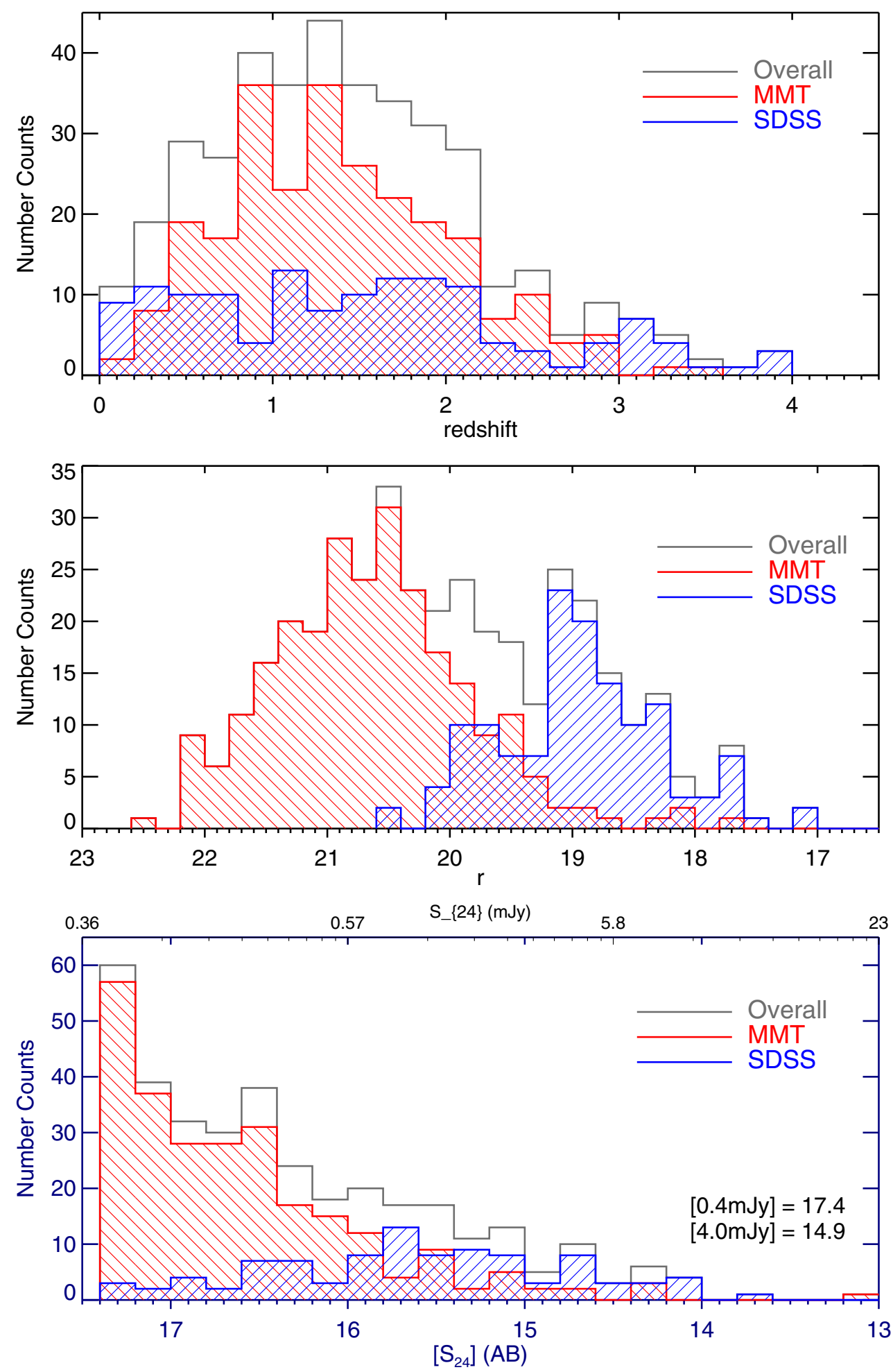

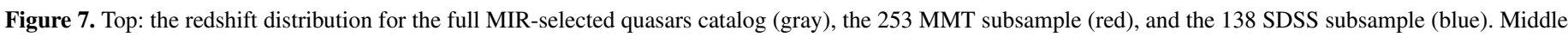

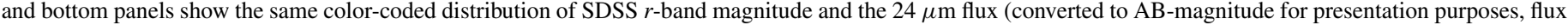
conversion examples are given in the bottom right).

(A color version of this figure is available in the online journal.)

difference in the optical-IR color $r-\left[S_{24}\right]$. At $r>20$, we also notice a very red population $\left(r-\left[S_{24}\right]>4.8\right)$ of MIR-selected quasars (inside the dashed line, Figure 8). The emergence of such a population may simply be a result of the fainter magnitudes the MMT sample covers, although this red population is still rare, comprising $14 \%$ of the $r>20$ MIR-selected quasars (32 out of 218). The absolute $i$-band magnitude $\left(M_{i}\right)$ for the red objects has a mean $M_{i}$ of -23.6 , which is one dex lower than the mean for the whole MIR-selected population $\left(M_{i}=-24.7\right)$.

We further examine the SDSS and MMT subsamples in the luminosity-redshift space (Figure 5). The majority (66\%) of the newly identified MMT quasars are fainter than the SDSS magnitude cut of $i=20.2$. A total of 93 MMT quasars also meet the SDSS magnitude limit $(i<20.2)$, which almost doubles 


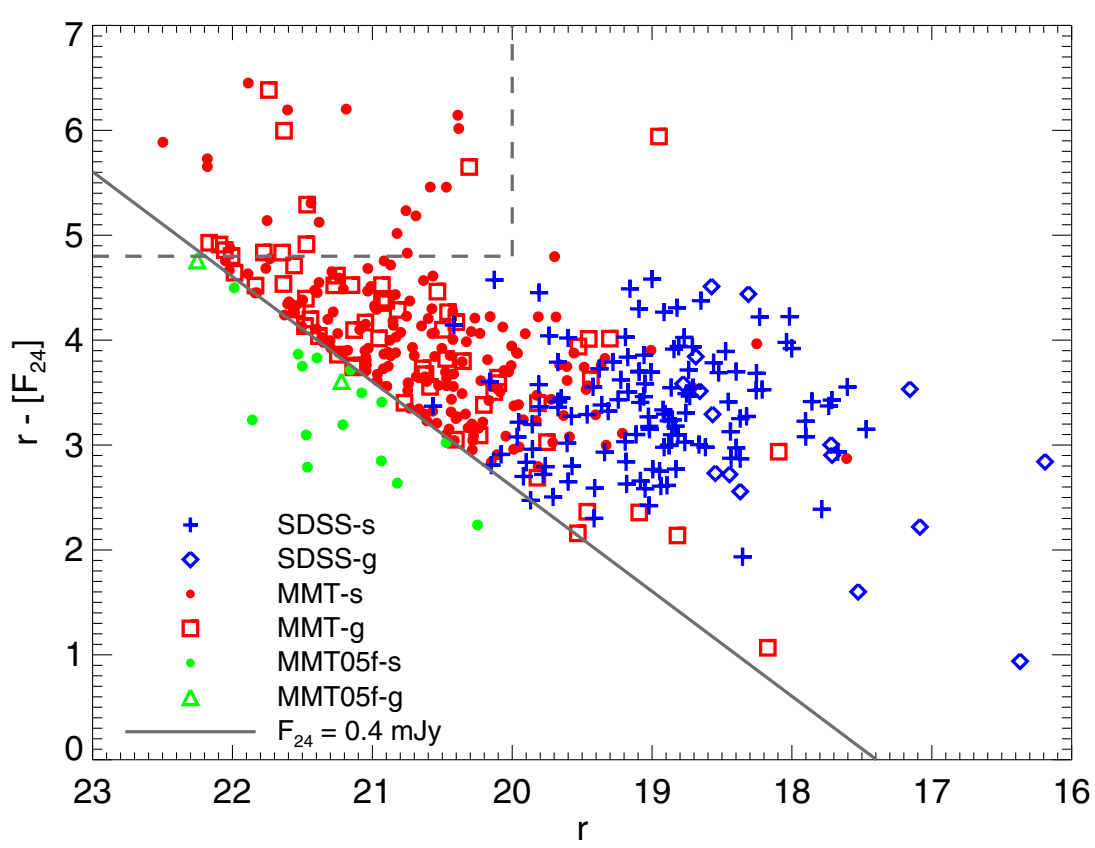

Figure 8. Comparison of different subsamples in terms of their MIR to optical $\left[r-\left[S_{24}\right]\right]$ colors: SDSS (blue), MMT (red), and MMT05-faint (green). In gray we mark the $24 \mu \mathrm{m}$ flux limit of $400 \mu \mathrm{Jy}$. Objects with point source morphology are the blue pluses (SDSS), red dots (MMT), and green dots (MMT05f); extended sources are marked by blue diamonds (SDSS), red squares (MMT), and green triangles (MMT05f). The morphologies are defined by the extendedness of the SDSS optical photometry; see Section 2.7.1. The different subsamples show similar MIR to optical colors within the range of [2, 4.8]. At $r>20$, a very red population of MIR-selected quasars emerges (dashed region, $r>20, r-\left[S_{24}\right]>4.8$ ), comprising a small fraction of 29 objects (14\%) out of the $212 r>20 \mathrm{MIR}$-selected quasars. (A color version of this figure is available in the online journal.)

the number of SDSS quasars in this region. One MMT source (2009.0131-268) at $z=3.537$ has an extremely high luminosity at $M_{i}=-29.97$. Such high luminosity is also rare in the SDSS catalog —only 82 quasars $(0.078 \%)$ in the 105,783 SDSS DR7 quasars are at $M_{i}$ brighter than -29.9. This quasar has consistent magnitudes at $i=17.5$ in modeled, fiber, and Petrosian SDSS magnitudes, but was missed in the SDSS DR7 quasar catalog for unknown reasons. The number densities of $z<3$ quasars is $\sim 10 \mathrm{deg}^{-2}$ at $i<19.1$, which is slightly higher than the $\sim 9 \mathrm{deg}^{-2}$ at $19.1<i<20.2$. The majority (78) of the new quasars are at $19.1 \leqslant i<20.2$ and $z<3$, which is a region the SDSS selection deliberately avoided to ensure the selection of high $z$ targets in their griz colors selection. At first glance this appears to be a major challenge to the SDSS's claim of $90 \%$ completeness to $i_{\mathrm{AB}}=20.2$. In the following section we will explore the reasons for this inconsistency.

\subsection{MIR Additions to the SDSS Quasar Selection}

The MIR-selected quasars are BEL (type 1) objects satisfying the joint limits of $r<22.5$ in the optical and $S_{24}>400 \mu \mathrm{Jy}$ in the MIR. The limit of $r<22.5$ is roughly equivalent to $i<22.4$ at $z \sim 1.5$, based on the Richards et al. (2006a) SED template. In Figure 5, 93 new quasars have been identified by the MMT spectroscopy above the SDSS DR7 quasar sample limit $(i \leqslant 20.2), 87$ of which also satisfy the SDSS magnitude limit of $M_{i}<-22$. Another six quasars are identified by reexamining the SDSS spectra. In this section we study why these objects were missed in the SDSS quasar catalog, as well as which additional objects the MIR selection is adding to the overall quasar population.

\subsubsection{Comparing the Selection Criteria}

The SDSS spectroscopic targets are selected primarily via color selection with the SDSS photometry (Richards et al. 2002a, R02), which includes the main low-z ugri, high- $z$ griz color selections, and a few other selections in the color-color or color-magnitude space: a mid- $z(2.5<z<3)$, two high- $z$, UVX, and $u g r$ outlier inclusion regions. The two main uniform color selections correspond to the two magnitude cuts at $i \leqslant 19.1$ (ugri) and $19.1<i \leqslant 20.2$ (griz), with the latter designed to recover high $z(z>3)$ targets only-certain conditions are set to exclude low- $z$ objects. In both magnitude bins, SDSS rejected targets that fell in the color boxes of white dwarfs, A stars, M stars, and white dwarf pairs. The SDSS selection also excludes objects in the $2 \sigma$ wide region around the stellar locus, with an exception for low $z$ resolved AGNs (Schneider et al. 2010). Therefore, only in the brighter $i \leqslant 19.1$ bin would extended sources be included, whereas at fainter magnitudes $(i>19.1)$ all SDSS targets are point sources. Secondary SDSS targets came from the Far-Infrared and Submillimetre Telescope radio source catalog (White et al. 1997) and ROSAT X-ray sources (Anderson et al. 2003). Color-colorselected SDSS targets were qualified as quasars if they were spectroscopically confirmed as BEL objects or showed interesting absorption features (Schneider et al. 2010).

The exclusion of extended sources in the high- $z$ griz color selection was achieved via the SDSS star-galaxy morphology separation. This separation is based on comparing the small point-spread function (PSF) magnitude and the larger exponential or de Vaucouleurs magnitude resulting from their different apertures. Objects for which the difference between the PSF and the modeled (exponential or de Vaucouleurs profiles) magnitudes is greater than $0.145 \mathrm{mag}$ are classified as extended ("galaxy-like," type = 3, R02); otherwise they are classified as point-source ("star-like," type = 6, R02).

The MMT targets in the MIR quasar sample, on the other hand, are only selected based on the $24 \mu \mathrm{m}$ flux limit and $r$-band magnitude cuts, before they are identified as BEL objects. The SDSS quasar selection criteria are necessarily much more 
Table 3

Number Counts of MIR-selected Quasars Identified Using SDSS and MMT Spectra in the Overlapping Regions

\begin{tabular}{lccccccc}
\hline \hline Magnitude & $N_{\text {SDSS }}$ & Ext & Point & $N_{\text {MMT }}$ & Ext & Point & Total Ext \\
\hline$i \leqslant 19.1$ & $(62+6)^{*}$ & $(4+6)^{*}(15 \%)$ & 58 & 13 & $7(54 \%)$ & 6 & $17(21 \%)$ \\
$19.1<i \leqslant 20.2$ & 27 & 0 & 27 & 80 & $22(28 \%)$ & 58 & $22(20 \%)$ \\
$i>20.2$ & 1 & 0 & 1 & 160 & $36(23 \%)$ & 124 & $36(22 \%)$ \\
& & & & $(133)^{\dagger}$ & $(25)$ & $(108)$ & \\
\hline Total & 96 & $10^{*}(10 \%)$ & $86(90 \%)$ & 253 & $65(24 \%)$ & $188(76 \%)$ & $75(21 \%)$ \\
\hline
\end{tabular}

Notes. The second line in each magnitude bin shows the number of new objects that also satisfy the SDSS selections (see also Section 2.7.2). Classification of the "Extended" (ext) and "Point-source" (point) morphological types are based on the SDSS photometry (Section 2.7.1). Throughout all magnitude bins, a constant $20 \%$ of the MIR quasars are extended sources.

* Six (6) are the newly identified BEL objects with SDSS spectra not in the DR7 quasar catalog, all of which are extended.

$\dagger$ For the objects that would satisfy the SDSS selection at brighter magnitudes, but no redshifts or point-source cut was applied.

complicated given the large sky density of $i<20.2$ objects (Section 2.6). As a result, the quasar detection rate is higher for the SDSS spectra $(\sim 16 \%)$, than in the MMT spectra $(\sim 10 \%$, Table 1).

Table 3 summarizes the number counts in three different $i$ magnitude bins and SDSS photometric types for the SDSS and MMT quasars in this sample. We found a constant fraction of $20 \%$ of "extended" MIR-selected MMT quasars in all magnitude bins, with the majority $(>80 \%)$ at lower $z(<1)$ and lower luminosity ( $\log L_{\text {bol }}<45.5 \mathrm{erg} \mathrm{s}^{-1}$, see also Section 5). These extended objects were automatically rejected in the SDSS selection at $i>19.1$. A second significant MIR addition comes from the fainter sources in the MMT surveys: a total of 160 objects are found at $i>20.2$, which SDSS did not cover.

\subsubsection{MIR Additions to the SDSS Completeness}

In this section, we compare the colors and photometric morphologies of the SDSS and MMT identified quasars in three different magnitude bins.

The SDSS uniform color selections have an estimated completeness based on simulated quasars, to be over $90 \%$ at $0<z<5.3$ down to $i=20.2$ (see also Table 6 in R02). This is an average completeness for previously known quasars, and applies to $i<19.1$ quasars at $z<2.5$, and to $i<20.2$ quasars at $3.5<z<5.3$. A later calibration of the completeness of the SDSS DR5 quasar survey gives an end-to-end completeness of $\sim 89 \%$ (Vanden Berk et al. 2005), which was confirmed in the SDSS DR5 quasar paper as "close to complete" for $0.7<z<1.0$ and $1.9<z<2.1$ at $\log \left(L_{\text {bol }}\right)\left(\mathrm{erg} \mathrm{s}^{-1}\right)>45.9$ and $>46.6$, respectively (Richards et al. 2006b; Shen et al. 2008).

The distribution of quasars in the $\sim 22 \mathrm{deg}^{2}$ LHS field is plotted in Figure 9. For a fair comparison, we focus only on the $\sim 12 \mathrm{deg}^{2}$ MMT covered region (within the circles and black polygon). There are a total of 96 SDSS quasars in the overlapping region (Table 3 ). Of these, 61 are uniformly colorselected (uniform flag $=1$ ), and 29 are selected by considering radio, $X$-ray, or other inclusion criteria (uniform flag $=0$ ). None of the SDSS quasars fall into the high- $z$-selected SDSS "QSO_ HiZ" branch (uniform flag = 2). As mentioned in Section 2.6, after re-inspecting the SDSS spectra we identified six additional quasars not included in the SDSS DR7 quasar catalog. They are represented by dark blue squares in Figure 5. There are 62 SDSS quasars at $i \leqslant 19.1,27$ quasars at $19.1<i \leqslant 20.2$,
Table 4

Number Counts of MIR-selected MMT Quasars that Fall in the SDSS Exclusion Zone

\begin{tabular}{lccr}
\hline \hline SDSS Exclusion & $N_{\text {total }}$ & Point & Ext \\
\hline M star + white dwarf & 20 & 13 & 7 \\
A star & 14 & 0 & 14 \\
White dwarf & 3 & 0 & 3
\end{tabular}

Notes. Numbers are accumulated values, for a breakdown in each magnitude bin, see the figure captions of Figures 10-12. "Point" and "Ext" refer to point-like sources and extended sources, respectively.

and 1 at $i>20.2$. MMT observations identify an additional 13 MMT09 and 6 SDSS quasars at $i \leqslant 19.1$, of which 10 MMT09 and 4 SDSS objects satisfy the SDSS $M_{i}=-22$ limit. At $19.1<i \leqslant 20.2,73$ MMT09 and 7 MMT05b quasars are added, of which 70 MMT09 and 7 MMT05b also satisfy $M_{i}<-22$ (Figure 5 and Table 3).

We first examine the bright magnitude bin of $i \leqslant 19.1$, where the SDSS ugri color selection is optimized for low $z(z<2.9)$ quasar selection and includes both extended and point sources. At $i \leqslant 19.1,15 \%$ of the SDSS quasars are extended ("galaxylike;" see Section 2.7.1), whereas in the MMT additions $~ 50 \%$ are extended (Table 3). In Figure. 10, we compare the MIRselected MMT and SDSS quasars at $i \leqslant 19.1$ in the four color-color and color-magnitude spaces. The majority of both MMT and SDSS samples fall inside the contours of 100 or more (thick curve) SDSS DR7 quasars per 0.1 magnitude bin. Only 4 of the 62 previously identified SDSS quasars are extended ("SDSS-g," marked as open blue diamonds in Figure 10). All six newly identified SDSS BEL objects (blue filled squares) are extended. They may have been rejected in the SDSS selection for being extended with blue $u-g$ colors (as indicated by vectors in Figure 4 of R02).

In the bright $i \leqslant 19.1$ bin, 9 of the 13 new MMT09 detections satisfy the SDSS selections, including 4 point sources and 5 extended sources at $z<2.6$ (Figure. 10, Table 3). The remaining four MMT quasars would have been rejected in the SDSS selection, since three are fainter than $M_{i}=-22$, and one point source falls in the SDSS M star + white dwarf exclusion region (marked by the magenta dashed lines in Figure 10, see also Table 4). Despite lying at the edge of the bulk of the SDSS contours, all nine new MMT objects have photometries that meet the $5 \sigma$ and error $<0.2$ requirement 


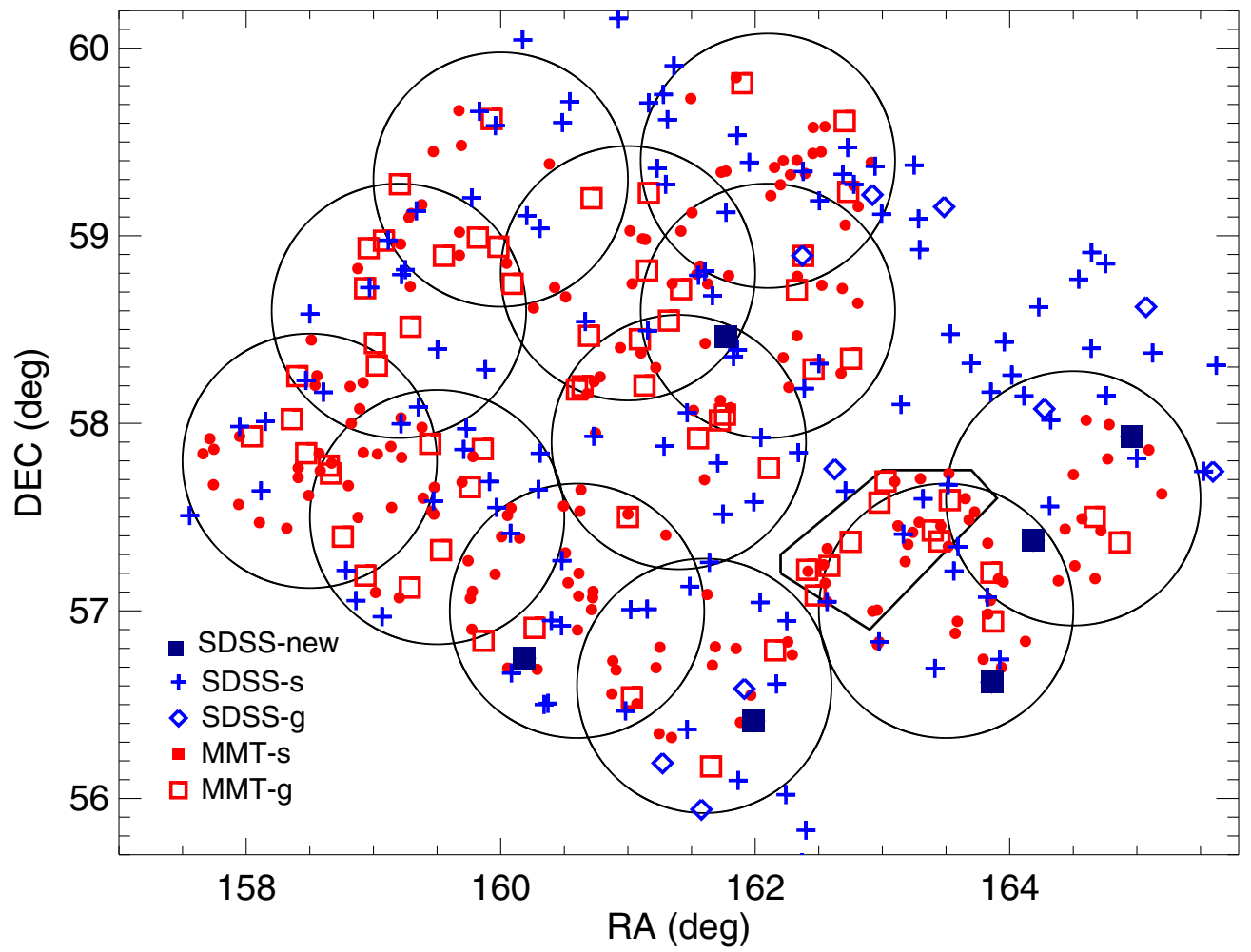

Figure 9. Quasar distribution in the LHS field: Black circles, the 12 MMT09 FOV; Black polygon, contour for the eight MMT05 FOV; Blue pluses, point-source quasars identified with SDSS spectra (SDSS-s); Blue diamonds, extended quasars identified with SDSS spectra (SDSS-g); red dots, point-source quasars identified with MMT spectra (MMT-s); red squares, extended quasars identified with MMT spectra (MMT-g). Navy squares, the six extended quasars identified with SDSS spectra, but not included in the SDSS DR7 quasar catalog (SDSS-new). For the definition of the photometric morphology see Section 2.7.

(A color version of this figure is available in the online journal.)

of the SDSS selection (R02). After adjusting for the MMT optical spectroscopy completeness (30\% for MMT09 and 66\% for MMT05b), the overall completeness of the SDSS selection at $i<19.1$ is $(67 \pm 8) \%$. Errors are Poisson estimates based on the inverse square root of total number of objects. This is $20 \%$ lower than the simulated $90 \%$ from R02.

In the fainter $19.1<i \leqslant 20.2 \mathrm{bin}$, SDSS applied different griz color cuts to select high $z$ ( $>2.9)$, point source targets. In this magnitude bin, MMT discovered 80 new objects (73 MMT09 and 7 MMT05b), the majority of which are at $z<2.9$ and are outside the SDSS-selected $z$ regions (R02). Of the two MMT objects that qualify the SDSS $z$ cut, only one is a point source and could have be added to the SDSS completeness analysis. Therefore, it is still valid to consider the SDSS selection complete to $\sim 90 \%$ at $z>2.9$ (Table 3). Most (>90\%) of the low- $z$ MMT quasars lie within the contours defined by the SDSS DR7 quasars and satisfy the SDSS color-color selections, although $\sim 30 \%$ of them are extended and would have been rejected had SDSS explored this low- $z$ regime (Figure 11).

In the faintest end $(i>20.2)$, which is below the SDSS quasar selection magnitude limit, only one SDSS quasar was included in the DR7 catalog ("52411-0947-531," not color-color selected, uniform flag = "0"). All 160 MMT quasars are newly identified objects. If compared to SDSS quasars at the brighter ends $(i \leqslant 20.2)$, the fainter targets show a large scatter in all colors (Figure 12), including 25 MMT sources in the SDSS exclusion zones (marked by dashed lines in the first 3 panels of Figure 12, Table 4): 13 in the M star + white dwarf exclusion region, 9 of which are extended sources; 9 in the A star exclusion zone, all of which are point sources; 2 in the white dwarf exclusion zone, both of which are point sources; and 1 point source in the white dwarf and A star overlapped exclusion region. Two other extended objects failed the $M_{i}$ cut. All the remaining 133 targets satisfy the SDSS magnitude and griz or ugri color selections, but not the $z$ or point-source constraints (Table 3). As with brighter magnitudes, a significant fraction (22\%) of the MIR quasars are extended, of which $\sim 70 \%(25 /$ 36) lie at $z<1$.

In Figure 13, we present the measured completeness of the SDSS quasar selections as a function of redshift, only taking into consideration the MMT objects that would otherwise satisfy the SDSS magnitude $\left(M_{i}<-22\right)$, redshift $(z<2.9$ at $i<19.1$, and $z>2.9$ at $19.1 \leqslant i<20.2$ ), color (ugri at $z<2.9$ and griz at $z>2.9$ ), and morphology (point source only at $i>19.1$ ) requirements: nine at $i \leqslant 19.1$ and one at $19.1<i \leqslant 20.2$ (Table 3). SDSS quasar selection is close to complete at $19.1<i \leqslant 20.2$ and $z>2.9$, but is overestimated by $\sim 20 \%$ at $i<19.1$ and $z<2.9$. The modified SDSS completeness is summarized in Table 5. These values are corrected for the spectroscopic completeness of the MMT survey-numbers are multiplied by 3.3 for MMT09 quasars and 1.5 for MMT05b objects. The corrections could be overestimated given the higher priority assigned to brighter $24 \mu \mathrm{m}$ objects, although it is unlikely to be by a significant number, as similar detection rates are found between MMT09 (30\% complete, $9.1 \%$ detection rate) and the more complete MMT05b survey (66\% complete, $9.9 \%$ detection rate).

\subsubsection{What Makes a Complete Quasar Sample?}

Several factors contribute to the MIR additions to the quasar population and the biases in the SDSS quasar selection. Table 3 summarizes the number counts in the two magnitude bins 

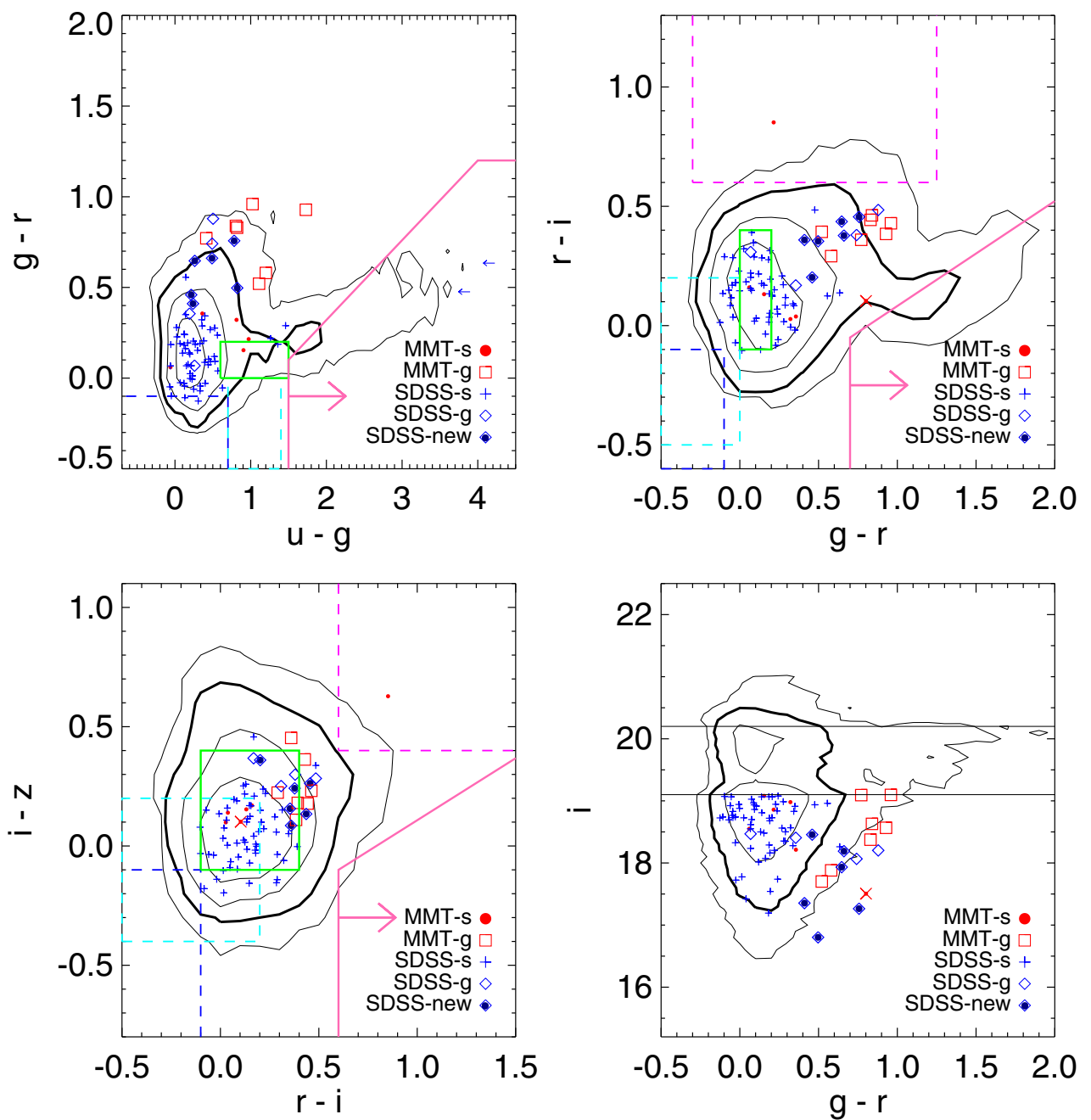

Figure 10. Location of SDSS (blue) and MMT (red) identified quasars in the SDSS color and magnitude diagrams for objects at $i \leqslant 19.1$. Symbols signals their SDSS photometric classification: blue pluses and red dots for SDSS and MMT point source ("-s," "star like"); blue diamonds and red squares for extended sources ("-g," "galaxy like"). The blue filled diamonds are the six newly identified SDSS BEL objects, which all have extended photometry. The bright MMT source ("2009.0131-268") is marked with a red cross in the center. Contours mark the distribution of the 110,509 SDSS DR7 quasars from Shen et al. (2011) at number densities of 20, 100, 500, and 1000 per 0.1 mag or color bin. The contour level of 100 objects per 0.1 magnitude bin is highlighted as a thick line to guide the eye. For objects with $<3 \sigma$ detections in either band, an upper/lower limit is used in the color-color plots. Dashed boxes are the different SDSS exclusion regions: blue for white dwarfs; cyan for A stars; magenta for M stars + white dwarfs. Solid boxes are: green, the mid- $z$ inclusion regions; solid magenta with an arrow, high- $z$ inclusion regions (unique in each panel, see Richards et al. (2002a)). The black lines in the " $g-r$ " vs. " $i$ " panel show the two SDSS magnitude cuts at $i=19.1$ and $i=20.2$. About half of the MMT subsample are extended sources, mostly covered by the outmost contour level of 20 objects per bin, and one point MMT source falls into the SDSS M star and white dwarf exclusion region.

(A color version of this figure is available in the online journal.)

Table 5

Observed SDSS Completeness of MIR-selected Quasars

\begin{tabular}{lcccccccc}
\hline \hline Apparent Magnitude & \multicolumn{7}{c}{ Redshifts } \\
\cline { 2 - 9 } & $0-0.5$ & $0.5-1$ & $1-1.5$ & $1.5-2$ & $2-2.5$ & $2.5-3$ & $3-3.5$ & $3.5-4$ \\
\hline$i \leqslant 19.1$ & 100.0 & 100.0 & 72.8 & 77.8 & 59.1 & 26.8 & $\ldots$ & $\ldots$ \\
\multirow{3}{*}{$19.1<i \leqslant 20.2$} & $(100.0$ & 100.0 & 100.0 & 100.0 & 96.3 & 57.2 & 89.9 & $99.8)$ \\
& $\ldots$ & $\ldots$ & $\ldots$ & $\ldots$ & $\ldots$ & $\ldots$ & 62.3 & 100.0 \\
& $(0.0$ & 0.0 & 0.0 & 0.0 & 0.0 & 11.4 & 74.2 & $98.4)$ \\
\hline
\end{tabular}

Notes. Numbers are in percentage. The SDSS simulated completeness from Table 6 in Richards et al. (2002a) is given in parenthesis.

in which SDSS carried out their completeness analysis. At $i \leqslant 19.1$, and $19.1<i \leqslant 20.2$, the MMT surveys add 13 and 80 additional quasars to the SDSS quasar catalog, respectively. Careful comparison reduces the numbers to nine and one quasars that also qualify for SDSS selection (Table 3). If we assume a homogeneous number density across all redshifts
(R02), we find that the SDSS completeness is overestimated by an average of $20 \%$ in $i<19.1$ quasars at $z<2.9$ (reported to be $>90 \%$ in R02), but is comparable to the reported $90 \%$ for $i>20.2$ quasars at $3.5<z<5.3$ (Figure 13). This completeness assumption is not physical, however, given the known cosmic evolution of quasar number density (Hasinger 

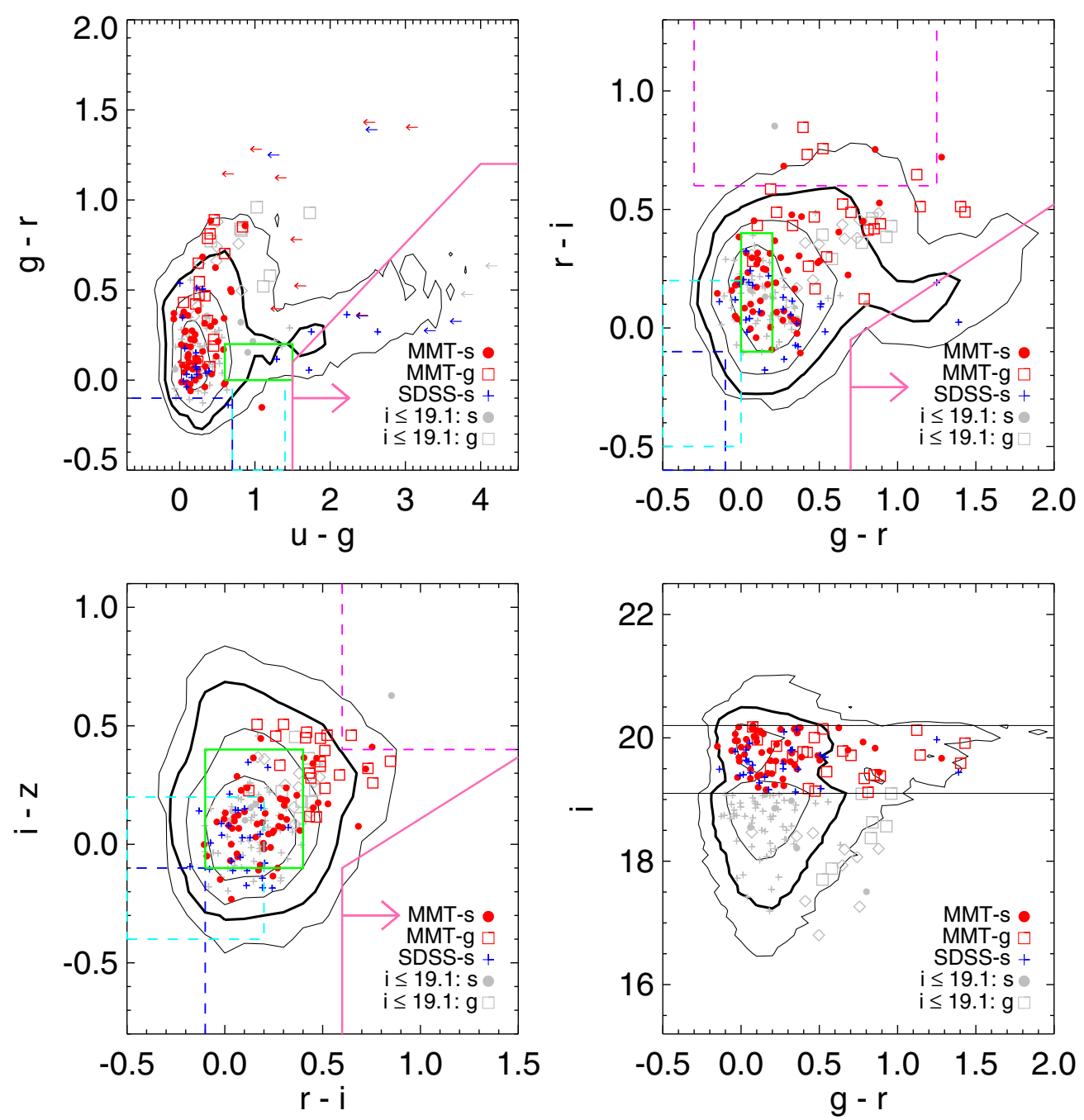

Figure 11. Colors and magnitudes of SDSS (blue) and MMT (red) identified quasars at $19.1<i \leqslant 20.2$ (including all redshifts). See Figure 10 for an explanation of the symbols and lines. In gray are the brighter objects from Figure 10, with point sources in dots and extended sources in squares and diamonds. The MMT-subsample shows a high fraction of extended sources (28\%). A total of 11 MMT quasars fall in the exclusion regions: six in the M star and white dwarf exclusion region, of which two are point sources; five in the A star exclusion region, all of which are point sources.

(A color version of this figure is available in the online journal.)

et al. 2005; Silverman et al. 2008), and therefore should be used with caution. Other MIR-selected samples, e.g., Lacy et al. (2013), did not show the completeness mismatch found in this paper. This is because color selections or wedges, both in optical and MIR, favor the power-law-shaped SEDs (Vanden Berk et al. 2001; Richards et al. 2002a; Lacy et al. 2004; Stern et al. 2005; Donley et al. 2012), and are biased against significant host galaxy contributions, the presence of strong emission lines (e.g., polycyclic aromatic hydrocarbon, $\mathrm{PAH}$ ), and other factors such as accretion rates (Ogle et al. 2006) and LINERs (Sturm et al. 2006). In contrast, the MIR flux limit applied in this sample selects everything above the corresponding luminosity, and therefore is not biased against dusty host galaxies or other factors mentioned above. In the whole $22 \mathrm{deg}^{2}$ LHS field, only six quasars in the SDSS catalog were rejected because of fainter MIR fluxes. The MIR flux-limited sample provides a complementary way to examine the quasar population as a whole, being more complete than the color selections. Of the MIR flux-limited quasars presented in this paper, the SDSS selection only recovers $58 \%$ and $10 \%$ of the total population at $i \leqslant 19.1$ and $19.1<i \leqslant 20.2$, respectively.
A significant fraction $(50 \%$ at $i \leqslant 19.1$, and $28 \%$ at $19.1<i \leqslant 20.2)$ of the newly identified MMT quasars are extended sources (Table 3 ). SDSS chose not to include extended sources at $i>19.1$ to avoid the contamination of very red, extended objects. Their choice was based on the observation that at $z \geqslant 0.6$, the majority of quasars are point sources. This point-source only selection turns out to be conservative, as $70 \%$ of extended targets at $i>19.1$ have a redshift higher than 0.6. Regardless of apparent magnitude, a constant fraction of $20 \%$ MIR quasars turn out to be extended sources (Table 3), although the majority $(80 \%)$ are of relatively low $z$ and luminosities $\left(z<1.5, \log \left(L_{\mathrm{bol}}\right)<45.5 \mathrm{erg} \mathrm{s}^{-1}\right.$, Figure 5, see also Section 5, Figure 24).

Another MIR addition to the sample arises from the SDSS cut of low- $z$ sources in the $19.1<i \leqslant 20.2$ bin (Figure 5). Because of this redshift cut, a significant number of quasars are missed from the sample, as the number density of $z<3$ quasars at $19.1<i<20.2$ is $\sim 24 \mathrm{deg}^{-2}$ (corrected for spectroscopic completeness) more than doubles the $\sim 10 \mathrm{deg}^{-2}$ found at $i<19.1$. Since the MMT09 survey is $30 \%$ complete (Section 2.3) and MMT05 is 66\% complete (Section 2.4), in 

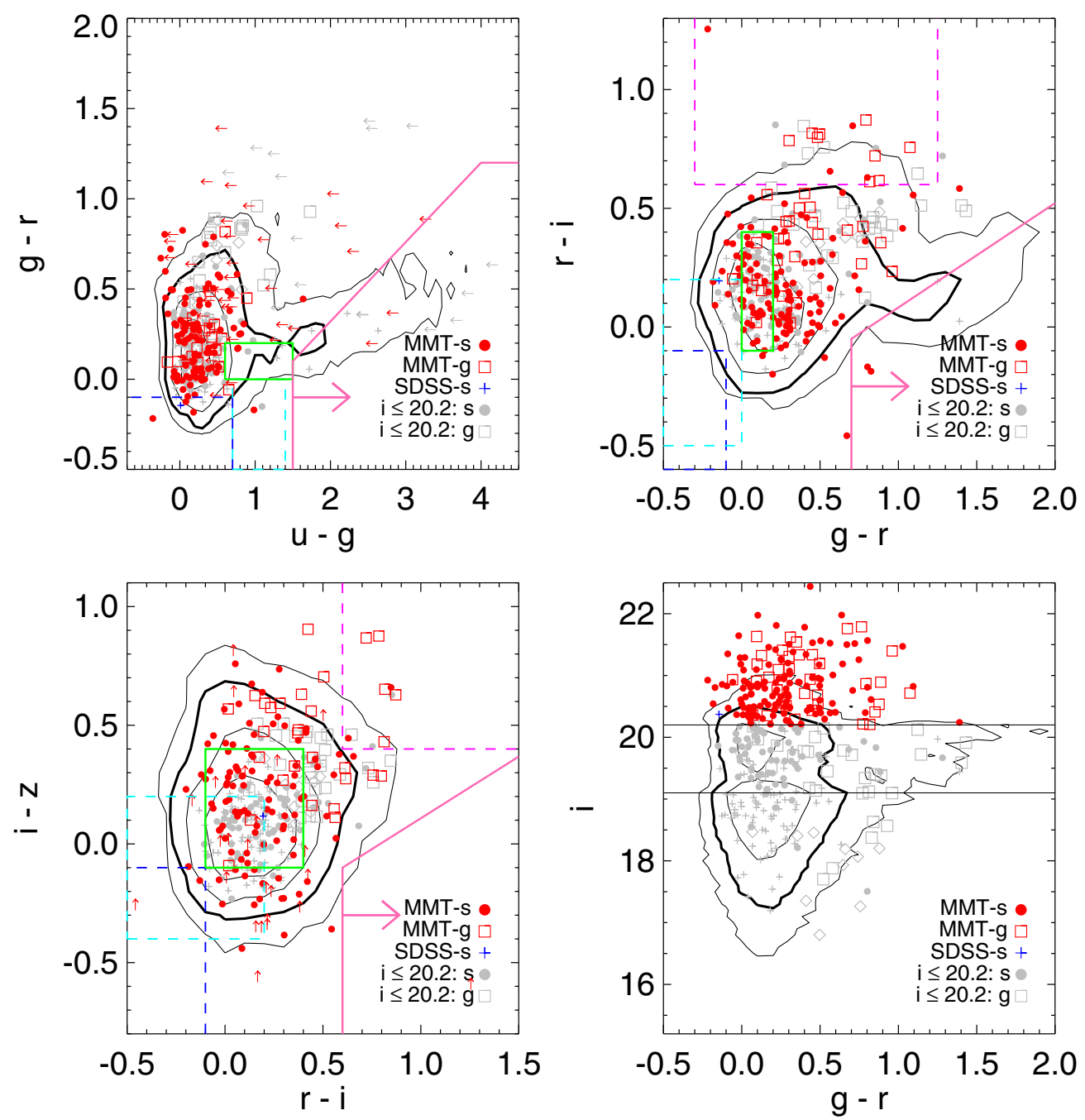

Figure 12. Colors and magnitudes of SDSS (blue) and MMT (red) identified quasars at the faint end of $i>20.2$. See Figure 10 and Figure 11 for an explanation of the symbols and lines. In gray are the brighter objects at $i \leqslant 20.2$. The MMT-subsample at $i>20.2$ consists of faint sources not covered by SDSS; and also shows a high fraction of extended sources (23\%). A total of 25 MMT quasars fall in the exclusion regions: four point sources and nine extended objects in the M star and white dwarf exclusion region; nine point sources in the A star exclusion region; two point sources in the white dwarf exclusion region; and one point source in the white dwarf and A star overlapping region. The remaining 133 objects also qualify for the SDSS color selection without $z$ or morphological cuts.

(A color version of this figure is available in the online journal.)

addition to the 80 newly identified MMT quasars, roughly 174 may remain undetected at $19.1<i \leqslant 20.2$. The majority $(90 \%)$ of the MMT quasars that fall into this $z<3$ region also satisfy the SDSS color selections.

The third MIR addition is the extension to faint targets $(i>20.2$; Table 3). The faint MIR quasars almost doubled the number of known quasars in this field, and the majority $(80 \%)$ also satisfy the SDSS color selections. The completeness corrected number density of $z<3$ quasars at $i>20.2$ is $\sim 45 \mathrm{deg}^{-2}$.

Finally, since the MIR selection does not avoid specific color areas-as compared to the SDSS exclusion regions of white dwarfs, M stars, and A stars-a total of 37 MMT quasars have been recovered (Table 4). They contribute to $\sim 10 \%$ of the total MIR quasar population. This is the fourth MIR addition to the SDSS quasar selection criteria.

\section{MEASUREMENTS OF SPECTRA}

Different virial SMBH mass $\left(M_{\bullet}\right)$ estimators have used different line width parameters-with either FWHM (in $\mathrm{km} \mathrm{s}^{-1}$ ) or line dispersion (i.e., the second moment of the emission-line profile). FWHM is easier and more straightforward to measure, but can be easily overestimated in cases of line blending or extended wings. Line dispersion $\left(\sigma_{l}\right)$, on the other hand, has relatively lower uncertainties, but may be overestimated for specific line profiles. Unfortunately, both parameters are affected by measurement errors, and can provide unreliable estimates for low S/N (<10) spectra (Denney et al. 2013). This problem can be circumvented via model fits, and Gaussian functions are widely used to fit the BELs. All the BH mass estimators we use (MD04, VP06, VO09, and S11) are based on either one or both the FWHM and $\sigma_{l}$ of the emission line. The line dispersion $\sigma_{l}$ is arguably more reliable, given the better consistency between different lines (Park et al. 2013; Denney et al. 2013), and the better scaling to the widely used empirical $M_{\bullet}-\sigma_{*}$ relation (Tremaine et al. 2002). Because the line broadening can be due to several components, a straightforward measurement of $\sigma_{l}$ is complicated, and for this work we decided to use the FWHM of the continuum subtracted emission line as the line width proxy. For a Gaussian, the FWHM has a simple correlation with $\sigma_{l}$, as FWHM $=2 \sqrt{2 \ln 2} \sigma_{l}$, or $2.35 \sigma_{l}$. If only one Gaussian is used, then the FWHM and $\sigma_{l}$ will be linearly 


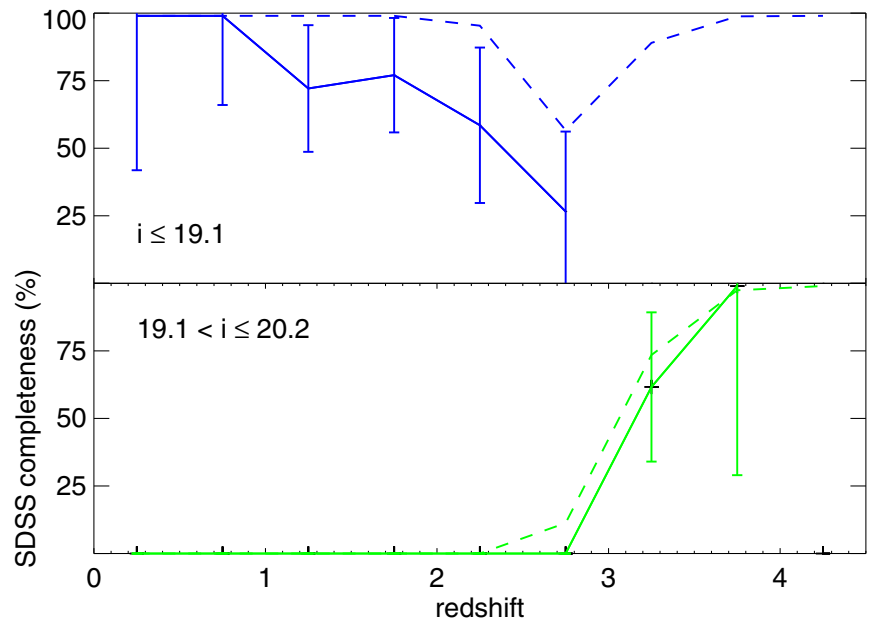

Figure 13. Observed SDSS quasar selection completeness after including qualifying MIR MMT quasars. The dashed lines plot the simulated completeness for SDSS quasars at a 0.5 redshift bin (Table 6 in Richards et al. 2002a). The SDSS completeness at $i \leqslant 19.1$ drops from an average $90 \%$ to $(67 \pm 8) \%$ for the MIR-selected quasars, but is comparable at $19.1<i \leqslant 20.2$.

(A color version of this figure is available in the online journal.)

correlated. If multiple Gaussians are used, the $\sigma_{l}$ will give a higher equivalent value than the dominant FWHM. We provide the $\sigma_{l}$ measurements in the machine-readable table.

We wrote an IDL procedure that first measures and subtracts the continuum, and then fits one or more Gaussian profiles to the emission line. The procedure is based on the code used for the SDSS quasar catalog (S11), but includes more generality. In cases where a single Gaussian is not a good fit to the line profile, up to three Gaussian components are allowed. An $F$-test is used to evaluate the need for each additional component. The $F$-test is widely used to compare the best fits of different models based on least squares comparison and the $F$ distribution. The $F$ value is computed as:

$$
F=\frac{\chi^{2}-\chi_{\text {new }}^{2}}{\mathrm{DOF}-\mathrm{DOF}_{\text {new }}} / \frac{\chi_{\text {new }}^{2}}{\mathrm{DOF}_{\text {new }}}
$$

where DOF is the number of degrees of freedom for the variance (Press et al. 1992). We compute the $F$-test values using the IDL mpftest program. ${ }^{12}$ In each case, we allow up to three Gaussians for the BEL and use an $F$-test confidence level of 0.999 as the threshold. Only in cases where the $F$-test threshold is met, which means the new fit is significantly different from the old one, will the extra broad component be kept. Figure 14 shows the fitting results of the same object with and without an $F$-test. This procedure differs from the SDSS approach, where as long as the new $\chi^{2}$ is smaller, an additional Gaussian component is added. Since the use of Gaussian profile(s) has no physical basis, we argue that the number of Gaussians should be minimized except in special cases (BALs and NALs; see Section 7).

The introduction of an $F$-test significantly decreases the number of Gaussian components needed for the emission line fits (Table 6). The percentage of objects that need more than one Gaussian component drops significantly from $94 \%$ to $6 \%$ for $\mathrm{H} \beta$; and from $74 \%$ to $29 \%$ for $\mathrm{Mg}$ II. However, for $\mathrm{C}$ IV, this percentage remains high at $76 \%$, partly due to the frequently observed asymmetry in the highly ionized C IV BELs.

\footnotetext{
12 http://cow.physics.wisc.edu/ craigm/idl/idl.html
}

Table 6

Number of Gaussians used in Fits with and without an $F$-test

\begin{tabular}{lcrr}
\hline \hline Emission Line & $N_{\text {Gaussian }}$ & $\begin{array}{c}N_{\text {obj }} \\
\text { without } F \text {-test }\end{array}$ & $\begin{array}{r}N_{\text {obj }} \\
\text { with } F \text {-test }\end{array}$ \\
\hline C IV & 1 & $30(21 \%)$ & $34(24 \%)$ \\
$(143)$ & 2 & $33(23 \%)$ & $66(46 \%)$ \\
& 3 & $80(56 \%)$ & $43(30 \%)$ \\
\hline Mg II & 1 & $75(26 \%)$ & $201(71 \%)$ \\
$(285)$ & 2 & $50(18 \%)$ & $77(27 \%)$ \\
& 3 & $160(56 \%)$ & $7(2 \%)$ \\
\hline $\mathrm{H} \beta$ & 1 & $8(10 \%)$ & $70(94 \%)$ \\
$(75)$ & 2 & $66(88 \%)$ & $4(5 \%)$ \\
& 3 & $1(2 \%)$ & $1(1 \%)$ \\
\hline
\end{tabular}

We measure the FWHMs in the quasar optical spectra for the main BELs: $\mathrm{H} \beta, \mathrm{Mg}$ II, and CIV. First, the continuum is fitted with a power law to the emission line-free region (Table 7). Fe II can be strong and broad due to many multiplets, especially in the vicinity of $\mathrm{Mg}$ II and $\mathrm{H} \beta$ lines. Therefore, the Fe II emission template is also used in the continuum fit for $\mathrm{Mg}$ II and $\mathrm{H} \beta$. The continuum fit wavelength windows are chosen such that there is no contamination from the tail of the BEL component. We adopt the optical Fe II template from Boroson \& Green (1992) for $\mathrm{H} \beta$, and the UV Fe II and Fe III templates from Vestergaard \& Wilkes (2001) for Mg II. No iron template is used for C IV, since the iron emission is generally weak in the $\mathrm{C}$ IV band. For $\mathrm{H} \beta$ and $\mathrm{Mg}$ II, the continuum and iron removal could be $\mathrm{S} / \mathrm{N}$-dependent. In cases where the $\mathrm{S} / \mathrm{N}$ of the spectra is limited (average $\mathrm{S} / \mathrm{N}$ per pixel $<4$ ), the iron line removal is not feasible, and for these objects we only fit a power-law continuum. This affects only $3 \%$ of the objects with a Mg II fit, and $8 \%$ of the objects with an $\mathrm{H} \beta$ fit.

Up to five parameters are fitted simultaneously for the continuum: continuum normalization $\left(A_{\lambda}\right)$ and continuum slope $\left(\alpha_{\lambda}\right)$; for $\mathrm{H} \beta$ and $\mathrm{Mg}$ II, Fe II template normalization $\left(A_{\mathrm{Fe}}\right)$, Fe II Gaussian line-width $\left(\sigma_{\mathrm{Fe}}\right)$, and Fe II velocity offset $\left(v_{\text {off }}\right)$ relative to the redshift. We then fit up to three Gaussians to the emission lines allowing for velocity offsets (BEL central wavelength), linewidth (FWHM and $\sigma_{l}$ ), and EW measurements. Each Gaussian is fitted with three parameters: maximum value (factor), mean value (central $\lambda$ ), and standard deviation $(\sigma)$. In the case of broad or asymmetric emission lines where multiple Gaussian components are used, we provide two sets of linewidths: the "dominant" FWHM, which is associated with the major component with the highest intensity, and the "non-parametric" FWHM, which is from the composite line profile. The dominant FWHM increases by an average of $\sim 30 \%$ after introducing the $F$-test, since fewer Gaussian components are used to reconstruct the emission line profile - this will increase the derived

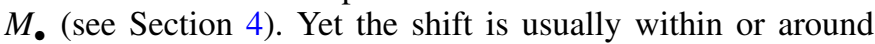
$1 \sigma$ of the FWHM error, and therefore the dominant FWHM after $F$-test is generally consistent with the values without the test.

Both NAL and BAL (FWHM > $1000 \mathrm{~km} \mathrm{~s}^{-1}$ ) are commonly observed in the C IV and Mg II BELs for MIR-selected quasars. NALs and BALs can affect the standard multiple Gaussian fitting algorithm and therefore need to be treated separately. If absorption features are observed, the spectra are manually fit individually. This approach is adopted to retrieve the line-width measurement as accurately as possible. Figure 15 shows an example of the absorption feature before and after the manual fit. Since the FWHMs of the emission lines are manually measured 

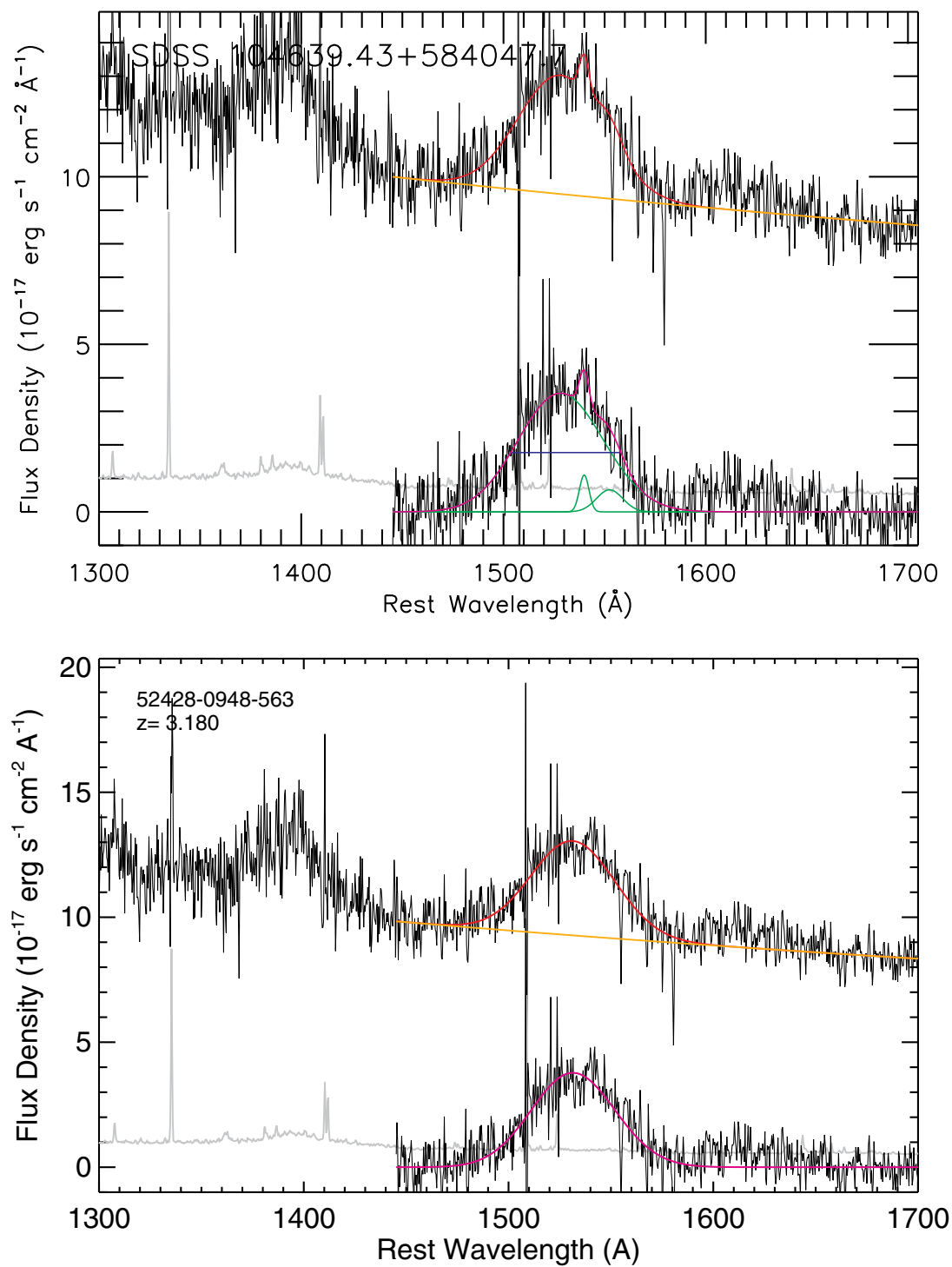

Figure 14. Example of a comparison of the C IV BEL fitting results with and without an $F$-test (smoothed over two pixels). The top panel shows the SDSS results from Shen et al. (2011), where no $F$-test was used and the emission line was fitted with three Gaussian components. The bottom panel shows the same object, but as the $F$-test shows a confidence level of 0.984 , we abandoned the additional Gaussian component and kept only a single Gaussian for the BEL profile. The same color codes as in S11 are used to guide the eye. Upper and lower black lines in each panel show the original and continuum-subtracted spectra. The gray line in the lower spectra is the flux-density errors. In orange is the continuum, covered by the composite spectra in red except for the emission line region. In green are the broad Gaussians used for the BEL (covered by the composite spectra in the bottom panel). The composite spectra of the emission line is in magenta. The S11 has a dominant Gaussian FWHM of $9728 \pm 506 \mathrm{~km} \mathrm{~s}^{-1}$, which is consistent with our results of $9409 \pm 282 \mathrm{~km} \mathrm{~s}^{-1}$. The equivalent width (EW) results are also consistent (S11: 21.7 \pm 1.4 ; this work: $20.3 \pm 1.4$ ). The additional Gaussian components in the SDSS fits are not necessary for this object.

(A color version of this figure is available in the online journal.)

Table 7

Wavelength Ranges used for Spectral Measurements in Rest Frame

\begin{tabular}{lcccc}
\hline \hline Emission Line & Redshift Range & $\begin{array}{c}\text { Continuum } \\
(\AA)\end{array}$ & Fe Template & $\begin{array}{c}\text { Emission } \\
(\AA)\end{array}$ \\
\hline $\mathrm{C}_{\text {IV }}$ & $1.63<z<4.39$ & {$[1445,1465]$ and $[1700,1705]$} & $\ldots$ & {$[1500,1600]$} \\
$\mathrm{Mg}_{\text {II }}$ & $0.43<z<2.10$ & {$[2200,2700]$ and $[2900,3090]$} & VW01 & {$[2700,2900]$} \\
$\mathrm{H} \beta$ & $z<0.76$ & {$[4435,4700]$ and $[5100,5535]$} & BG92 & {$[4700,5100]$} \\
\hline
\end{tabular}

Notes. The redshift ranges are the MMT and SDSS accessible ranges based on their secure spectral ranges (See Section 2.6). VW01, Vestergaard \& Wilkes (2001); BG92, Boroson \& Green (1992).

after subtracting the absorption features they lack error bars. They will be used for $M_{\bullet}$ analysis, but are flagged in the catalog. More discussion can be found in Sections 3.4 and 7, and in a forthcoming paper on the absorption features in MIR quasars (Y. S. Dai et al., in preparation).

\subsection{CIV}

The C IV line is fitted for the 143 objects with $1.63<z<$ 4.40. Iron contamination is not significant for C IV, thus, only a two parameter $\left(A_{\lambda}, \alpha_{\lambda}\right)$ power-law continuum fit is used. 

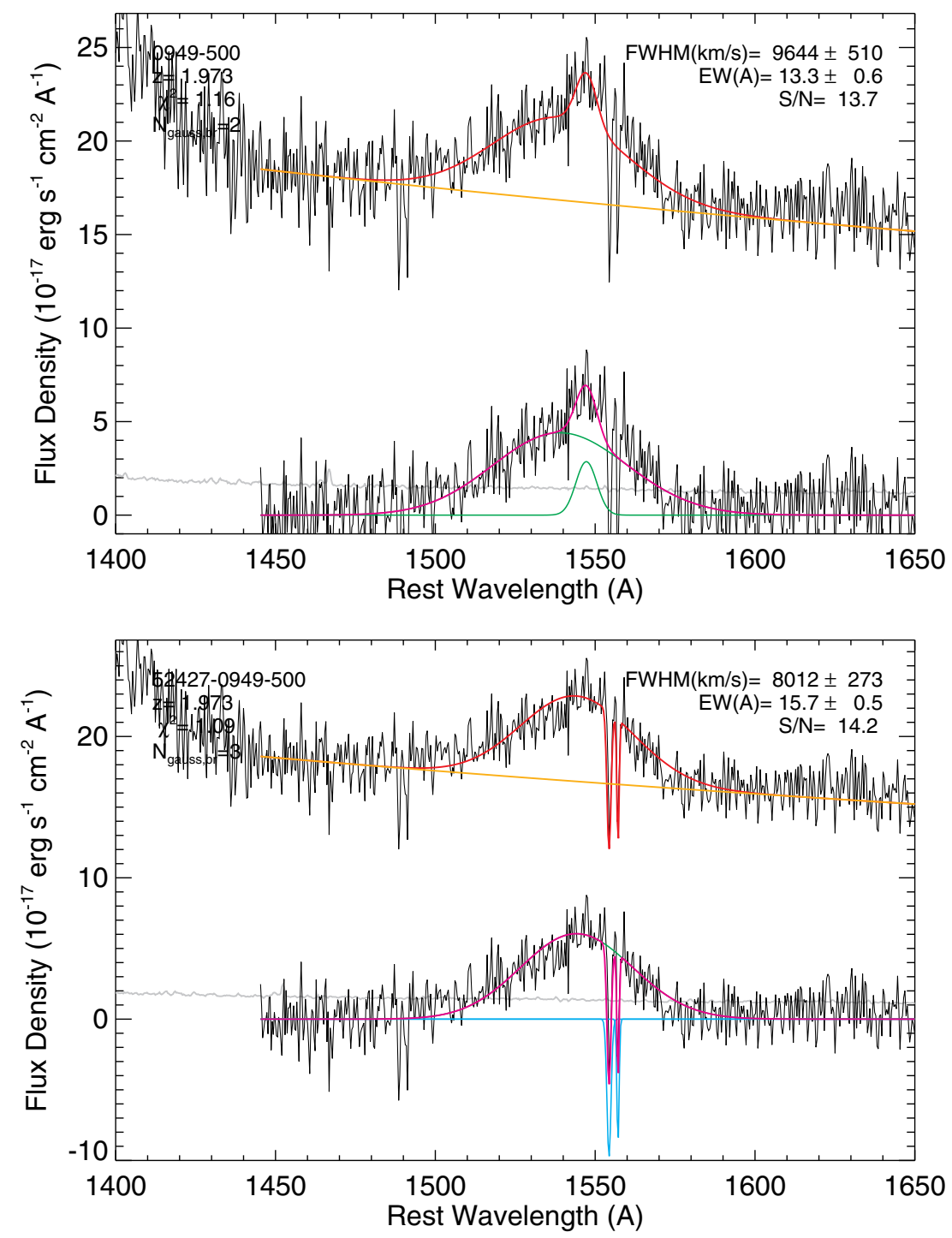

Figure 15. Example of a quasar with an automatically (top) and manually (bottom) fitted $\mathrm{C}$ IV line profile (smoothed over two pixels). The manual fit accounts for the absorption feature, and better constrains the FWHM of the BEL. Upper and lower black lines in each panel show the original and continuum-subtracted spectra. The gray line in the lower spectra is the flux-density errors. In orange is the continuum, covered by the composite spectra in red except for the emission line region. In green are the broad Gaussians used for the BEL, and in cyan the absorption feature- a C IV $\lambda \lambda 4959,5007$ doublet is clearly seen redshifted from the BEL peak. The composite spectra of the emission line is in magenta.

(A color version of this figure is available in the online journal.)

We subtract the continuum fit to the line-free regions, and then fit the C IV emission line (Table 7). We did not subtract a narrow $\mathrm{C}$ IV from the line profile because it is still debated as to whether a narrow C IV component is present (Wills et al. 1993; Marziani et al. 1996; Sulentic et al. 2007), and to be comparable with other studies (e.g.,VP06, S11, Assef et al. 2011; Park et al. 2013). For the same reason, we also did not fit the $1600 \AA$ feature (Laor et al. 1994; Fine et al. 2010). It is common (>70\%) that more than one Gaussian component is required (Table 6) to fit the $\mathrm{C}$ IV BEL profile: $48 / 61$ for SDSS, 56/75 for MMT09, and 5/7 for MMT05b. In $\sim 40 \%$ of the C IV emission lines, NALs or BALs are seen in or adjacent to the BEL profile. Figure 16 shows an example of a typical C Iv fit.

\section{2. $M g$ II}

The $\mathrm{Mg}$ II line is fitted for the 285 objects with $0.43<z<$ 2.10. We adopt the iron template from Vestergaard \& Wilkes (2001) and fit the continuum plus iron template to the emission- line free region (Table 7). In nine sources with $\mathrm{Mg}$ II coverage, the iron template is not constrained due to low spectra quality $(\mathrm{S} / \mathrm{N}$ per pixel $<4)$, in which only the power-law continuum was subtracted. When the $\mathrm{Mg}$ II emission line is fit, the $\mathrm{Mg}_{\text {II }} 2796$, $2803 \AA$ doublet $\left(\sim 750 \mathrm{~km} \mathrm{~s}^{-1}\right.$ at rest-frame) is not taken into account given the much greater FWHM of the Mg II emission line in all cases. As it is still debatable whether a narrow $\mathrm{Mg}$ II component should be removed from the BEL profile (McLure \& Dunlop 2004; Vestergaard \& Osmer 2009; Wang et al. 2009), we provide measurements with a single Gaussian for the narrow component $\left(<1200 \mathrm{~km} \mathrm{~s}^{-1}\right)$. Objects that need multiple broad components are $\sim 30 \%$ (Table 6): $25 / 81$ for SDSS, $53 / 183$ for MMT09, and 6/21 for MMT05b. NALs are seen in $\sim 8 \%$ of the objects. Figure 17 shows an example of a typical $\mathrm{Mg}$ II fit.

$$
\text { 3.3. } H \beta
$$

The $\mathrm{H} \beta$ line is fitted for the 75 objects with $z<0.76$. We adopt the iron template from Boroson \& Green (1992) and 


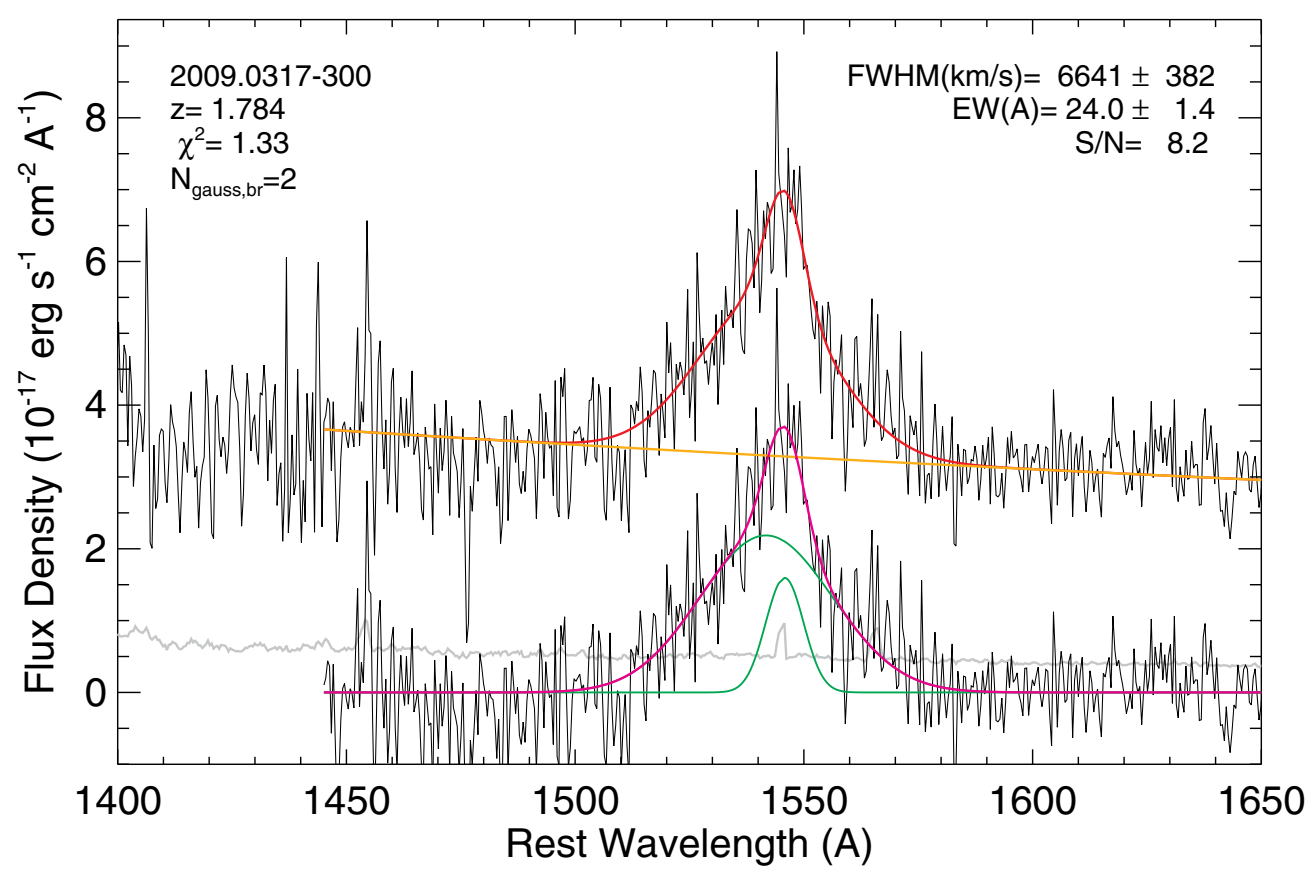

Figure 16. Example of the spectral fit for C Iv BEL (smoothed over two pixels). Upper and lower black lines show the original and continuum-subtracted spectra. Top left shows the redshift, $\chi^{2}$ of the fit, and the number of Gaussians used in the broad line fits; top right is the fitting results of the dominant FWHM, EW, and median $\mathrm{S} / \mathrm{N}$ of the emission line region. In gray is the flux-density errors. In orange is the continuum, covered by the composite spectra in red except in the BEL region. In green are the Gaussian components for the BEL. The composite spectra of the emission line is in magenta. The "dominant" FWHM is from the broader Gaussian in green, while the "non-parametric" FWHM is from the composite line profile in magenta. As shown in this case, the "dominant" FWHM is commonly broader than the "non-parametric" FWHM in 70\% of the targets with multiple Gaussians.

(A color version of this figure is available in the online journal.)

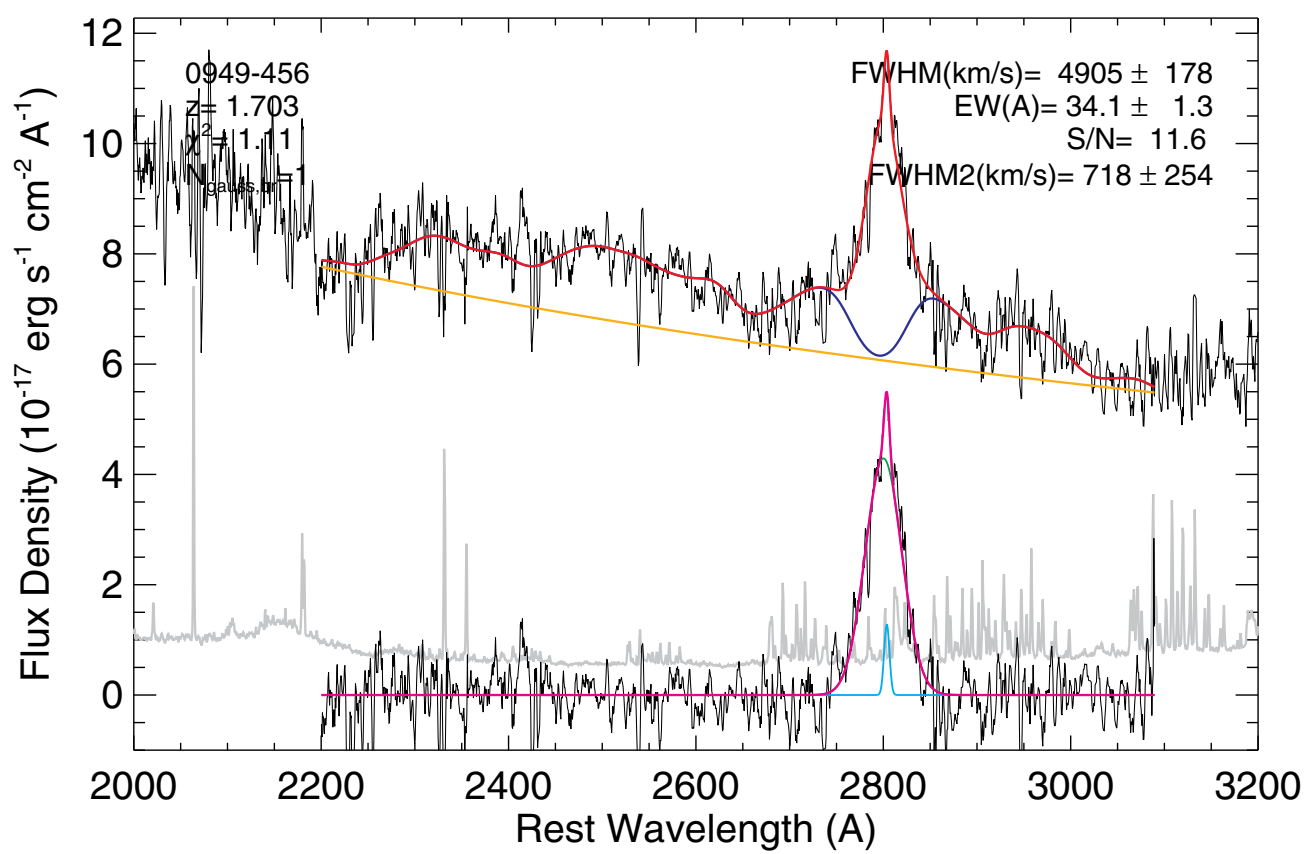

Figure 17. Example of the spectral fit for Mg II BEL (smoothed over two pixels). Upper and lower black lines show the original and continuum and Fe templatesubtracted spectra. Colors and legends are explained in Figure 16, With the purple curve showing the $F e$ template, mostly covered by the composite spectra in red, except in the BEL region. In cyan is the Mg II narrow emission component (FWHM $<1200 \mathrm{~km} \mathrm{~s}^{-1}$ ), whose FWHM is marked by FWHM2 in the legend.

(A color version of this figure is available in the online journal.)

fit the continuum plus iron template in the designated spectral windows (Table 7). In four objects with $\mathrm{H} \beta$ coverage, the iron template is not constrained due to the low quality of the spectra $(\mathrm{S} / \mathrm{N}$ per pixel $<4)$, and only a power-law continuum was subtracted. After subtracting the continuum and iron emission lines, we fit the $[\mathrm{O}$ III] $\lambda \lambda 4959,5007$ doublets together with the
$\mathrm{H} \beta$ component. For the $\mathrm{H} \beta$ components, we allow up to three Gaussians to fit the BEL, and use a single Gaussian to account for each of the narrow $\mathrm{H} \beta$ and [O III] emission lines. We require the narrow $\mathrm{H} \beta$ component and the [O III] doublets to have the same velocity shift and broadening, and constrained their FWHM to $<1200 \mathrm{~km} \mathrm{~s}^{-1}$. Only in $<5 \%$ cases do we need multiple 


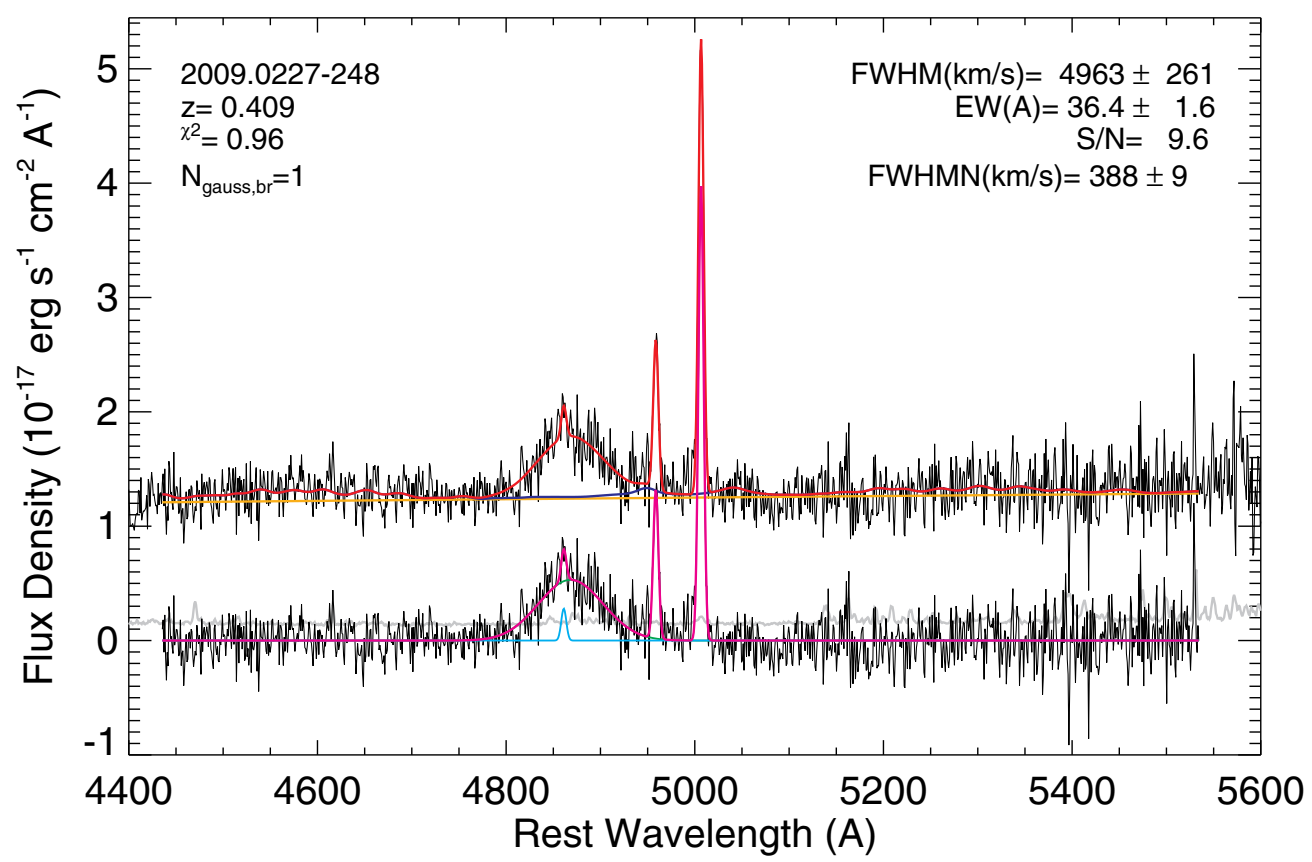

Figure 18. Example of the spectral fit for $\mathrm{H} \beta$ BEL (smoothed over two pixels). Upper and lower black lines show the original and continuum and Fe template-subtracted spectra. Colors and legends are explained in Figures 16 and 17. In cyan is the $\mathrm{H} \beta$, [O III] $\lambda \lambda 4959,5007$ narrow emission components, whose FWHM is marked by FWHMN in the legend.

(A color version of this figure is available in the online journal.)

Gaussians (Table 6): $3 / 38$ for SDSS, $2 / 31$ for MMT09, and 0/6 for MMT05b. Figure 18 shows an example of a typical $\mathrm{H} \beta$ fit.

\subsection{Uncertainties of Spectral Measurements and Error Estimates}

The uncertainties in the spectral measurement arise from three main sources: (1) the quality of the spectra and instrument errors; (2) the adopted fitting process (e.g., ambiguity introduced from using certain line profiles and from using one or multiple components); and (3) special features that could affect the algorithm, in particular, a narrow line component, especially for Mg II and C IV (see S11), instrumental broadening with BEL, or strong NALs or BALs.

The fitting errors based on $\mathrm{S} / \mathrm{N}$ are automatically accounted for through our IDL program using the IDL program mpfitfun.pro. ${ }^{13}$ This program returns the $1 \sigma$ errors of each parameter from the covariance matrix. The quality of the spectra directly affects the fitting results. We observed similar S/N dependences as in S11. The uncertainty in the FWHM and EW measurements increases as the $\mathrm{S} / \mathrm{N}$ in the line-fitting region decreases (Figure 19, top). Little or no influence from the continuum $\mathrm{S} / \mathrm{N}$ is found for the continuum fitting results (Figure 19, bottom).

Instrumental broadening is not a problem for the BEL. Hectospec has a spectral resolution of $170-380 \mathrm{~km} \mathrm{~s}^{-1}$ at the redshifts $(0<z<4)$ for the sample (Fabricant et al. 2008). The SDSS has a $1.5 \sim 2$ times higher resolution (Abazajian et al. 2009). For the BELs, $99 \%$ have FWHM $>2000 \mathrm{~km} \mathrm{~s}^{-1}$, so the instrumental resolution correction is negligible. However, the instrument resolution is comparable to the observed NAL widths (a few hundred $\mathrm{km} \mathrm{s}^{-1}$ ), so that instrumental broadening must be removed. We used the formula $\mathrm{FWHM}_{\text {measured }}^{2}=\mathrm{FWHM}_{\text {intrinsic }}^{2}+$ $\mathrm{FWHM}_{\text {instrument }}^{2}$ to correct the observed line-width for narrow

\footnotetext{
13 http://www.physics.wisc.edu/ craigm/idl/down/mpfitfun.pro
}

Table 8

Average FWHM Uncertainties in Our Sample and Comparison to the Literature

\begin{tabular}{lccccc}
\hline \hline Emission Line & This Work & S11 & VO09 & VP06 & P13 \\
\hline C IV $_{\text {IV }}$ & $26 \%$ & $21 \%$ & $6 \%$ & $9 \%$ & $6 \%$ \\
\hline $\mathrm{Mg}$ II & $20 \%$ & $27 \%$ & $10 \%$ & & \\
\hline $\mathrm{H} \beta$ & $25 \%$ & $27 \%$ & $12 \%$ & $10 \%$ & \\
\hline
\end{tabular}

Notes. VP06, Vestergaard \& Peterson (2006), 28 quasars (reverberation mapping, RM); VO09, Vestergaard \& Osmer (2009), 34 SDSS quasars and 978 LBQS quasars (single-epoch spectra, SE); S11, Shen et al. (2011), of 105,783 SDSS-selected quasars (SE); P13, Park et al. (2013), of 39 AGNs (RM). The uncertainty differences arise from spectral quality and the different methods used to measure them (Section 3.4).

absorption lines. The non-Gaussian, flat-topped fiber profile of MMT Hectospec (Fabricant et al. 2008) renders this correction imperfect and will be discussed in the absorption paper (Y. S. Dai et al., in preparation).

We adopt the Monte Carlo flux randomization method as in the SDSS routine (S11). This approach provides a more reasonable estimate than from the program fit alone, as it also smoothes out the ambiguity of whether or not to subtract a narrow line for C IV or Mg II BELs. We generate 50 mock spectra with the same wavelength and flux-density error arrays as the original spectrum, and randomly scatter the flux values with Gaussian noise (allowing negative values) based on the original errors. We then apply the same fitting procedure described in Section 3. The measurement uncertainties are defined as the standard deviation of the measured parameters in the 50 mock spectra. This uncertainty is on average 2.1, 2.9, and 3.6 times larger than the fitting errors in FWHM for $\mathrm{H} \beta, \mathrm{Mg}$ II, and $\mathrm{C}$ IV , respectively. The average FWHM uncertainties are summarized in Table 8. The uncertainties given in VP06 were adopted as the largest fitting error from their five continuum settings and could be underestimated, as the single fitting error is on average 

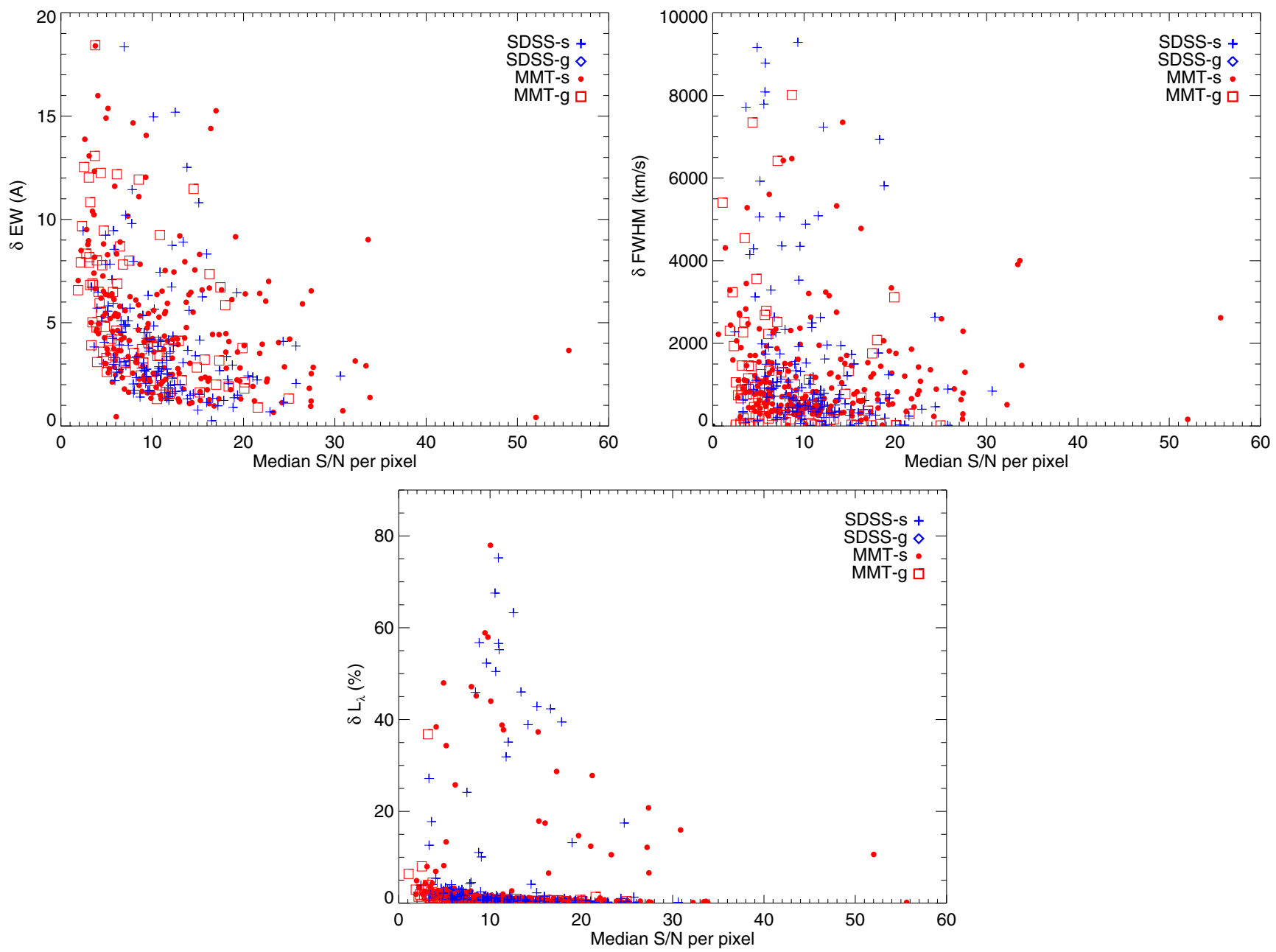

Figure 19. Uncertainties in the EW and FWHM measurements (top) and the continuum fitting results (bottom), vs. the median $\mathrm{S} / \mathrm{N}$ per pixel of the fitting region. Color codes are explained in Figure 9. We observe decreasing uncertainties for EW and FWHM as the S/N of the spectrum increases, but the S/N influence on the continuum fitting is not obvious.

(A color version of this figure is available in the online journal.)

two to three times lower than using the Monte Carlo method. The average scaling factor between single fit and Monte Carlo uncertainties is then used to scale the uncertainties of FWHM and EW in $\sim 100$ lines with strong absorption features.

The errors in FWHM and continuum measurements will directly affect the final SMBH mass (Section 4). A 50\% uncertainty in FWHM translates to a 25\% uncertainty in SMBH mass. In general, the flux-density and spectral measurement errors are in the range of $20 \%-30 \%$. For the SDSS subsample, our error estimates generally agree with the SDSS results.

\section{VIRIAL BLACK HOLE MASSES}

The SMBH mass is one key property in studying the SMBH-host connection. Among the various $M_{\bullet}$ estimators (e.g., Kormendy \& Richstone 1995; Gebhardt et al. 2000; Marconi \& Hunt 2003), the virial mass estimate is one of the simplest and most adopted (e.g., Kaspi et al. 2000; McLure \& Dunlop 2004; Vestergaard \& Osmer 2009). The virial method is a powerful tool, especially in the absence of host galaxy information, where stellar velocity dispersion or bulge luminosity is missing. The virial method is based on the assumption that the dynamics in the vicinity of the nucleus, the "broad-emissionline-region (BLR), are dominated by the gravity of the SMBH, so that the mass of the central SMBH can be estimated from the virialized velocity of the line-emitting gas. The virial method based on the emission lines is calibrated by reverberation mapping (RM) results, which use time delays measured from the BEL variability (e.g., Vestergaard \& Peterson 2006; Wang et al. 2009; Park et al. 2013). In the RM method, the BLR radius can be measured via the light travel time delayed response of the emission line flux to the continuum variation. However, only a few dozen objects have reliable RM masses due to the demanding exposure and $\mathrm{S} / \mathrm{N}$ requirements (Denney et al. 2013). The virial method is more commonly used as it requires only singleepoch (SE) spectra. For SE spectra, the BEL line-width is used as direct proxy for the SMBH mass, based on the assumption that the BLR radius is proportional to the luminosity-the observed R-L relationship (VP06; Collin et al. 2006; Bentz et al. 2009) - and the BEL line-width is proportional to the Keplerian velocity of the accreting gas.

The virial mass estimators for SMBH based on SE spectra are usually expressed as:

$$
\log \left(\frac{M_{\bullet}}{M_{\odot}}\right)=a+b \log \left(\frac{\lambda L_{\lambda}}{10^{44} \mathrm{erg} \mathrm{s}^{-1}}\right)+c \log \left(\frac{\mathrm{FWHM}}{\mathrm{km} \mathrm{s}^{-1}}\right)
$$

where $M_{\odot}$ is the solar mass. The term $\lambda L_{\lambda}$ is the continuum luminosity, which is a proxy for the BLR radius (Kaspi et al. 2000; Bentz et al. 2006, 2013). They are measured from chosen 
Table 9

Frequently Used Virial Black Hole Mass Estimators

\begin{tabular}{lcccc}
\hline \hline Emission Line & $\begin{array}{c}\text { Continuum } \lambda \\
(\AA)\end{array}$ & $\mathrm{a}$ & $\mathrm{b}$ & Reference \\
\hline $\mathrm{H} \beta$ & 5100 & 0.672 & 0.61 & MD04 \\
$\ldots$ & $\ldots$ & 0.910 & 0.50 & VP06 \\
\hline $\mathrm{Mg}$ II & 3000 & 0.505 & 0.62 & MD04 \\
$\ldots$ & $\ldots$ & 0.860 & 0.50 & VO09 \\
$\ldots$ & $\ldots$ & 0.740 & 0.62 & S11 \\
\hline C IV & 1350 & 0.660 & 0.53 & VP06 \\
\hline
\end{tabular}

Notes. MD04: McLure \& Dunlop (2004); VP06: Vestergaard \& Peterson (2006); VO09: Vestergaard \& Osmer (2009); S11: Shen et al. (2011). The sets of estimators we used for the fiducial SMBH mass are bold.

wavelengths close to each BEL (Table 9). The coefficients $a$ and $b$ are empirical values based on the SMBH masses from RM and a comparison among different lines. The coefficient $c$ normally has a fixed value of 2. Since the BEL line width (FWHM) represents the virial velocity, this factor of 2 exemplifies the virial nature of the $\operatorname{BLR}\left(M_{\bullet} \propto G v^{2} R^{-1}\right)$. Recently, a few papers have suggested using other values for $c$, based on the comparison of SE and RM results. For instance, Wang et al. (2009) used 1.09 and 1.56 in front of the $\mathrm{H} \beta$ and $\mathrm{Mg}$ II FWHMs, respectively. Park et al. (2013) used 0.56 in front of the C IV FWHMs. If a $<2$ factor is adopted, the resulting SMBH mass estimate will be smaller accordingly. Here, we stick to the $c=$ 2 value to be consistent with the SDSS quasar catalog (S11).

The $\mathrm{C}_{\mathrm{IV}}, \mathrm{Mg}$ II, and $\mathrm{H} \beta$ BELs are widely used as virial $\mathrm{BH}$ mass calibrators (e.g., McLure \& Dunlop 2004; Vestergaard \& Peterson 2006; Vestergaard \& Osmer 2009; Shen et al. 2011). We summarize the most frequently used virial estimators in Table 9. If multiple Gaussian components are used, in the catalog we provide both the dominant and the non-parametric $M$. derived from the dominant and non-parametric FWHM. In the following analysis of $M_{\bullet}$ properties for the $\mathrm{Mg}$ II and $\mathrm{H} \beta$, we use the $M_{\bullet}$ derived from the non-parametric FWHM to be consistent with the literature definitions. This choice of non-parametric FWHM in general provides lower $M_{\bullet}$ estimates than from dominant FWHM, and may underestimate the $M$ • for BELs if the emitting gas is in Keplerian motion.

For the CIV calibrator, the line-width definition in the literature is also the same as the non-parametric FWHM (VP06; see also Peterson et al. 2004). However, it is debated as to whether it provides a reliable $M_{\bullet}$. estimate due to the large scatter between the generally consistent $\mathrm{C}$ IV and $\mathrm{H} \beta$ derived M. (Netzer et al. 2007; Assef et al. 2011). This scatter may result from non-virial components from outflows or winds in the C IV BLR (e.g., Richards et al. 2011). For this MIR-selected quasar sample, we find a marginally better correlation between the dominant C IV FWHM and the non-parametric Mg II FWHM (Figure. 20, left). Better consistency is also found between the $M$. derived from the dominant $\mathrm{C}$ IV component and Mg II BELs (Figure. 20, right), indicating a non-virial contribution in the non-parametric BEL profile. Based on the correlation results, we choose to use the dominant C IV FWHM for $M_{\text {e }}$ estimates. We will discuss the choice and its implications in Section 7.

In our catalog, if applicable, we present multiple $M_{\bullet}$, using MD04 (H $\beta$, Mg II), VP06 (H $\beta$, C IV), VO09 (Mg II), and S11 (Mg II) estimators. We attribute the $M_{\bullet}$ from MD04 (H $\left.\beta\right), \mathrm{S} 11$ (Mg II), and VP06 (C IV) as the "fiducial" $M_{\bullet}$ to each object, because the $M_{\bullet}$ from these parameters are best-correlated with each other (Figure 21, left). We compare the different estimators based on the subsample of quasars that have two BELs with a median $\mathrm{S} / \mathrm{N}$ per pixel of $>5$ and no BAL/NAL, which leaves 20 objects with both $\mathrm{Mg}$ II and $\mathrm{H} \beta$ BELs, and 38 targets with both $\mathrm{C}$ IV and Mg II BELs. The comparison of the $M_{\bullet}$ from different lines and estimators for quasars with two BELs is achieved by forcing a linear correlation and measuring the $\chi^{2}$ values to compare the sample scatter.

We first compare the three Mg II estimators (MD04, VO09, S11) with the CIV estimator (VP06), and found a marginally smaller $M_{\bullet}$ scatter for VP06 (C IV) and S11 (Mg II) $\left(\chi^{2}=1.07\right)$ than for VP06 (C IV) and VO09 (Mg II $)\left(\chi^{2}=1.07\right)$. Both have a $\chi^{2}$ value $\sim 1$ dex better than VP06 (C IV) and MD04 (Mg II). The slope coefficient in all three sets of estimators agree with each other within errors at a value $\sim 0.6$. The scatter in $\log \left(M_{\mathrm{BH}}, \mathrm{C} \mathrm{IV}\right.$, (VP06) $/ M_{\mathrm{BH}}, \mathrm{Mg}$ II, (S11)) is similar to the scatter for the SDSS DR7 catalog (see Figure 10, S11). This small scatter between S11 and VP06 is by design, as the S11 coefficients were empirically adopted to provide the best correlation between VP06 (C IV) and S1 1 (Mg II) results. For ease of comparison with the SDSS sample, we assign the $M_{\bullet}$ from S11 as the fiducial $M_{\bullet}$ from $\mathrm{Mg}$ II BEL.

We then make the same $\chi^{2}$ comparison for the two $\mathrm{H} \beta$ estimators (VP06, MD04) and the chosen Mg II estimator S11. For the same $\mathrm{H} \beta$ BEL, $M_{\bullet}$ from VP06 is systematically 0.2 dex higher than from MD04, since the VP06 $a$ factor is $\sim 0.2$ larger (Table 9). S11 and MD04 show a slightly smaller scatter $\left(\chi^{2}=0.59\right)$ than S11 and VP06 $\left(\chi^{2}=0.78\right)$, so $M_{\bullet}$ from MD04 is chosen as the fiducial $M_{0}$ in $\mathrm{H} \beta$ BELs. The scatter in $\log \left(M_{\mathrm{BH}}, \mathrm{H} \beta,(\mathrm{MD} 04) / M_{\mathrm{BH}}, \mathrm{Mg}\right.$ II, $)(\mathrm{S} 11)$ is also similar to that of the SDSS DR7 catalog (see Figure 10, S11).

In summary, for the MIR-selected sample, we find that MD04 $(\mathrm{H} \beta), \mathrm{S} 11$ (Mg II), and VP06 (C IV) show the best correlations and assign a fiducial $M_{\bullet}$ using these three estimators. If $M_{\bullet}$ from $\mathrm{Mg}$ II and $\mathrm{H} \beta$ BELs are both available, the $M_{\bullet}$ derived using $\mathrm{H} \beta$ will be adopted as the fiducial $M_{\bullet}$ because of the robust SE mass scaling from $\mathrm{H} \beta \mathrm{RM}$ studies. For targets with $M_{\bullet}$ from both $\mathrm{C}$ IV and $\mathrm{Mg}$ II BELs, we attribute the $\mathrm{Mg}$ II derived $M_{\bullet}$ given the possible complications of non-virial component from the Civ BELs.

In Figure 21 (right), we plot the mass ratio distribution for the quasar subsample with two BELs (median $\mathrm{S} / \mathrm{N}$ per pixel of $>5)$. The mean and $1 \sigma$ from a Gaussian fit to the mass ratio distributions are $(0.01,0.34)$ for $\log \left(M_{\mathrm{BH}}, \mathrm{H} \beta\right.$, (MD04) $\left./ M_{\mathrm{BH}}, \mathrm{Mg} \mathrm{II},(\mathrm{S} 11)\right)$ and $(0.11,0.42)$ for $\log \left(M_{\mathrm{BH}}, \mathrm{C} \mathrm{IV}\right.$, (VP06) $/ M_{\mathrm{BH}}, \mathrm{Mg}$ II, (S11)). The mean offsets are negligible because they are smaller than what a typical FWHM error would introduce: a $30 \%$ error in FWHM translates to an upper and lower uncertainty of +0.11 dex and -0.15 dex in the $\log \left(M_{\bullet}\right)$ space, and justifies the choice of these three estimators.

We show the SMBH mass and redshift distribution for the MIR-selected quasar sample in Figure 22, and superpose samples from the literature for comparison. The redshift distribution of the MIR-selected quasars is typical of an apparent-magnitudelimited sample, and has a large overlap with the SDSS, BQS, and LBQS catalogs. For $M_{\bullet}$, the MIR-selected sample also overlaps with the above mentioned samples, but has a higher fraction of lower mass objects than the S11 sample-a direct result of the fainter magnitude limit applied.

\section{BOLOMETRIC LUMINOSITY AND EDDINGTON RATIOS}

We measure the bolometric luminosity $L_{\text {bol }}$ from the fitted spectra continuum luminosities: $L_{\text {bol }}=k \times L_{\lambda}$, where $L_{\lambda}$ are 

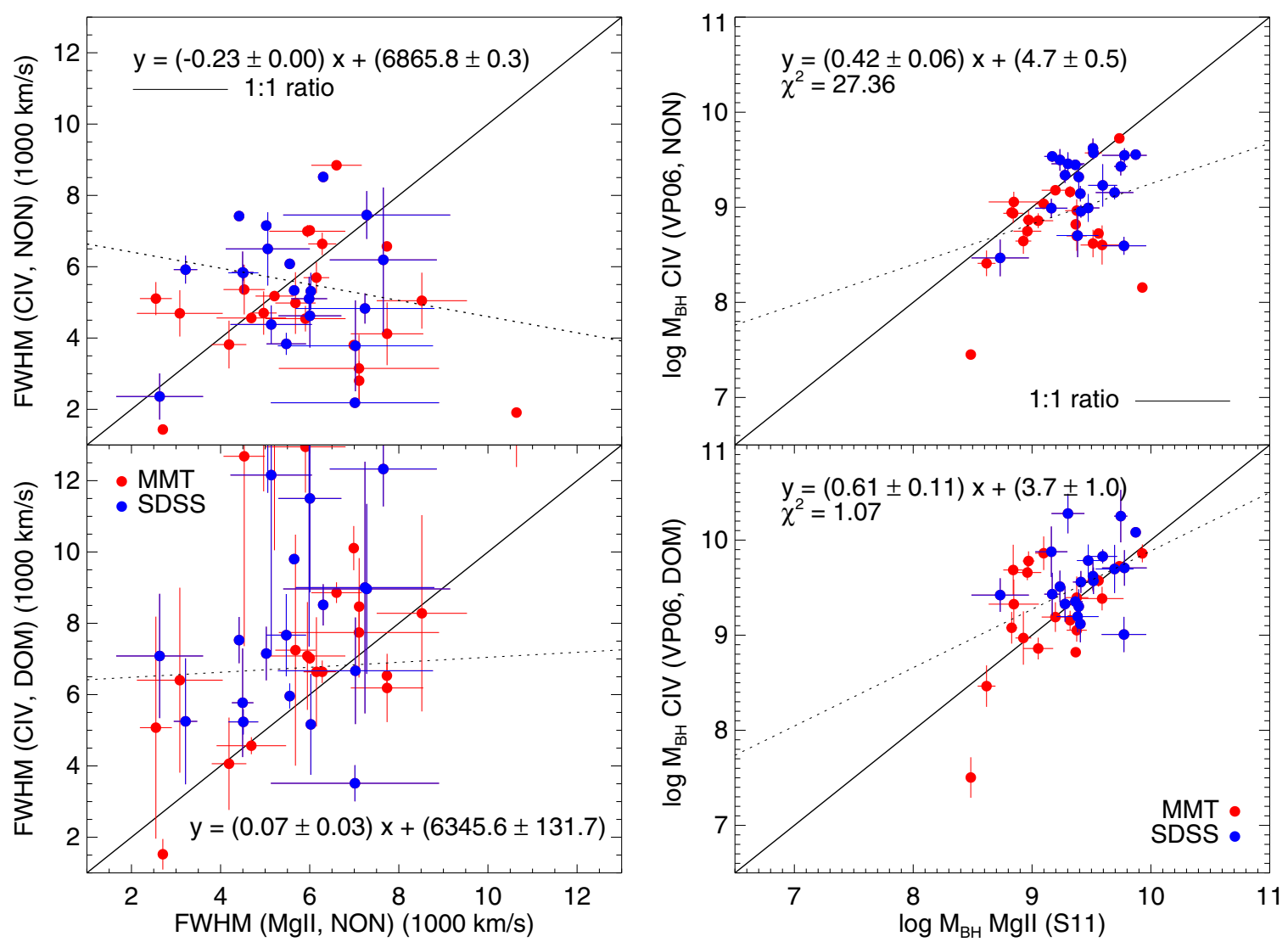

Figure 20. Left: comparison of the non-parametric (top) and dominant (bottom) C IV FWHM against the non-parametric Mg II emission line width. (right) SMBH mass $\left(M_{\bullet}\right)$ in $M_{\odot}$ derived from the non-parametric (top) and dominant (bottom) C IV FWHM against the $M_{\bullet}$ from the non-parametric Mg II FWHM. In each pane, diagonal lines mark the linear correlation (See Section 4). The MIR-selected MMT targets are in red and the SDSS targets in blue. The dominant C Iv FWHMs are systematically higher than the non-parametric C IV FWHM in 70\% of the cases with multiple Gaussians (see, e.g., Figure 16), and have a marginally smaller scatter from the 1:1 linear correlation with the $\mathrm{Mg}$ II non-parametric FWHM. A better correlation to the $\mathrm{Mg}$ II derived $M_{\bullet}$ is also observed of the $M_{\bullet}$ from dominant $\mathrm{C}$ IV FWHM (linear fit slope: $0.61 \pm 0.11$ ) than from the non-parametric C IV FWHM (linear fit slope: $0.42 \pm 0.06$ ), possibly indicating a non-virial component in the CIV BEL.

(A color version of this figure is available in the online journal.)

$L_{5100}(z<0.76, \mathrm{H} \beta), L_{3000}(0.43<z<2.10, \mathrm{Mg}$ II $)$, and $L_{1350}(1.63<z<3.18$, C IV $)$ in $\mathrm{erg} \mathrm{s}^{-1}$; and $k=9.26,5.15$, and 3.81, respectively (see S11). The coefficient $k$ values are from the composite SED from Richards et al. (2006a, R06), a modified SED largely consistent with Elvis et al. (1994). The R06 template should be applicable to at least the point source targets in this work, since it is based on 259 Sptizerdetected SDSS type 1 (BEL) quasars, 96\% (248/259) of which also qualify the MIR-selection of $S_{24}>400 \mu \mathrm{Jy}$ for this sample. Therefore, we caution the usage of the cataloged $L_{\mathrm{bol}}$ and its derived parameters for extended objects. We did not correct the spectra for intrinsic extinction (see also Section 2.5). This may result in $L_{\mathrm{bol}}$ being underestimated for systems with strong reddening; or overestimated if there is significant host contamination. A fourth estimator using $S_{24}$ flux shifted to the rest-frame is also introduced for comparison, in which the $k$ values differ from redshift to redshift. Given the uncertainty in the quasar MIR SED shapes (Dai et al. 2012), we caution the use of the MIR flux-derived $L_{\mathrm{bol}}$. It is on average 0.5 dex higher than the optical continuum-derived values, possibly from degenerate factors of reddening, host contamination, and sometimes PAH emission contamination at $z>2$. For comparison, we will only discuss the continuum-derived $L_{\mathrm{bol}}$ in the following discussion. All MIR-selected quasars have $L_{\text {bol }}$ greater than $10^{44} \mathrm{erg} \mathrm{s}^{-1}$, confirming their quasar nature (Figure 23).
For the MIR-selected SDSS subsample, a comparison with the SDSS DR7 quasar catalog (S11) shows consistency within $3 \sigma$ in continuum-derived $L_{\mathrm{bol}}$ (Figure 23 ) for over $80 \%$ of the MIR-selected targets. The MIR-selected quasars have an overall lower $L_{\text {bol }}$ distribution than SDSS DR7 quasars, since they include a large fraction (40\%) of objects fainter than the SDSS magnitude cut at $i>20.2$. The median fitting errors for $L_{\mathrm{bol}}$ are $2 \%, 1 \%$, and $3 \%$ for the $\mathrm{H} \beta, \mathrm{Mg}$ II, and C IV BELs, respectively. In objects that fall in $0.46<z<0.76$ or $1.63<z<2.10$, where two BELs are covered, we find a $\sim 40 \%$ consistency between the $L_{\text {bol }}$ from C IV and $\mathrm{Mg}$ II, and $\sim 15 \%$ between $\mathrm{Mg}$ II and $\mathrm{H} \beta$, which indicates reddening or host contribution. In the following analysis, if two $L_{\mathrm{bol}}$ are available for the same object, we use the $L_{\mathrm{bol}}$ that corresponds to the chosen $M_{\bullet}$ (see Section 4).

In Figure 24, we compare the $M_{\bullet}$ with $L_{\mathrm{bol}}$. The diagonal line marks the Eddington luminosity for the corresponding SMBH mass. Quasars rarely exceed $L_{\text {Edd }}$ (Kollmeier et al. 2006), and SDSS quasars tend to lie above $\sim 0.05 L_{\text {Edd }}$ and below a "subEddington boundary" (Falcke et al. 2004; Labita et al. 2009; Steinhardt \& Elvis 2010). Controversies exist as to whether the observed sub-Eddington limit is due to the incompleteness of the SDSS sample at low $M_{\bullet}\left(M_{\bullet}<3 \times 10^{8} M_{\odot}\right)$ and low Eddington ratios $\left(\mathrm{ER}, L / L_{\mathrm{Edd}}<0.07\right)$ (Kelly \& Shen 2013). For the MIR-selected sample, we do not observe a clear sub-Eddington limit (Figure 25). The $M_{\bullet}$ for MIR-selected 

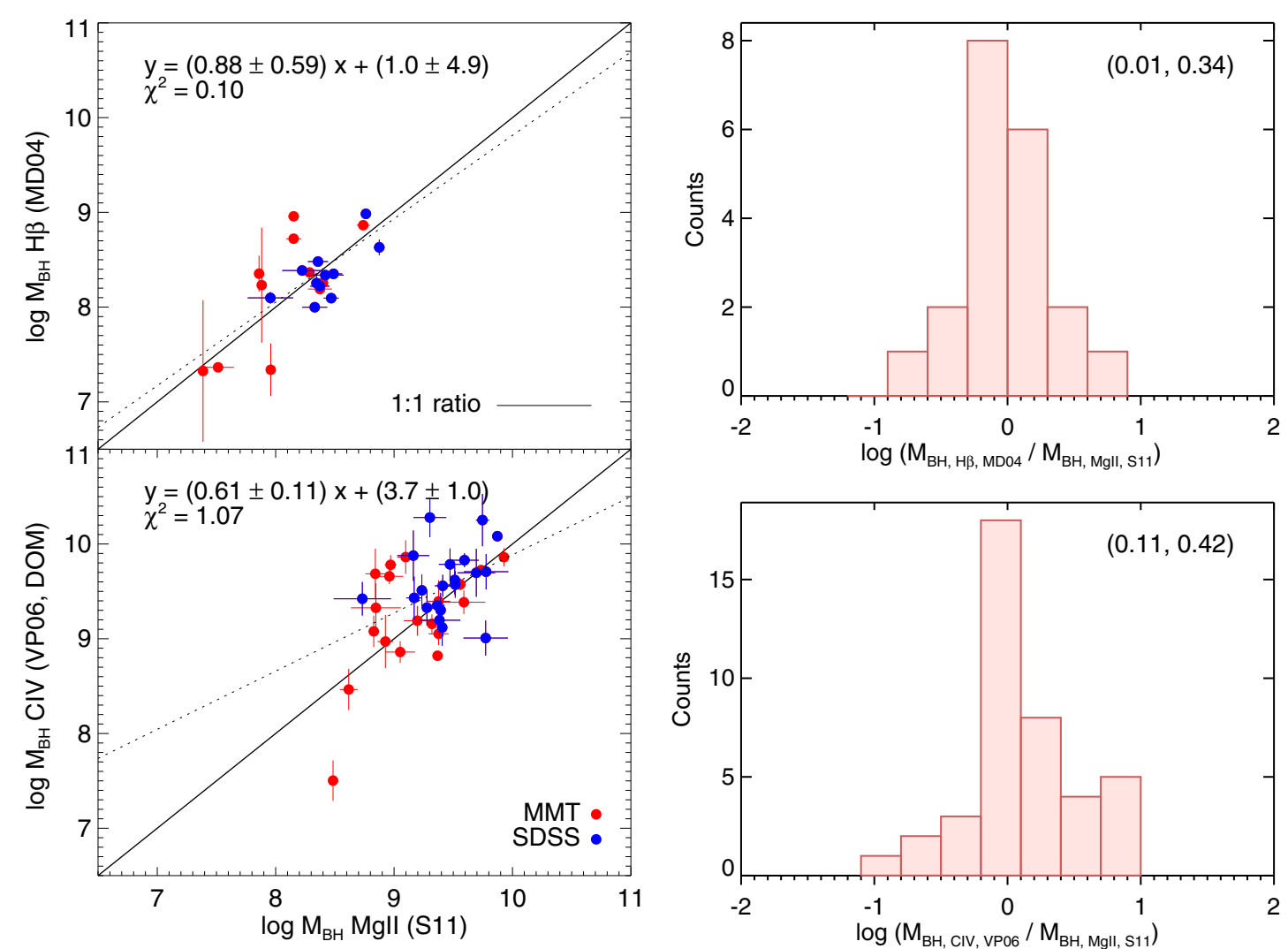

Figure 21. Left: comparison of SMBH masses in $M_{\odot}$ derived from different lines for the quasars with both $\mathrm{H} \beta$ and $\mathrm{Mg}$ II (top), Mg II and C IV (bottom) BELs. MMT sources are marked in red and SDSS sources in blue. A median line $\mathrm{S} / \mathrm{N}$ per pixel $>5$ was required. The diagonal line marks the 1:1 correlation, and the dashed line marks the best-fit linear correlation. The best-fit coefficient and associated errors are marked at the top left corner. The $M_{\bullet}$ from the S11 (Mg II) estimator shows a tight correlation with the $M_{\bullet}$ from MD04 $(\mathrm{H} \beta)$, and is consistent with the $M_{\bullet}$ from VP06 (C IV). (right): The mass ratio distributions for the two sets of estimates for the same object. A median line $\mathrm{S} / \mathrm{N}$ per pixel $>5$ was required. The mean and $1 \sigma$ from a Gaussian fit to the distribution are plotted at the top right corner.

(A color version of this figure is available in the online journal.)

quasars shows a trend of downsizing, although the $L_{\mathrm{bol}} / L_{\mathrm{Edd}}$ is relatively independent of redshift (Figure 26). These trends are similar to the results from the SDSS DR5 quasars (Labita et al. 2009). The wide span of Eddington ratios $(\sim 2 \mathrm{dex})$ is similar to that previously found in Woo \& Urry (2002). Table 10 summarizes the $M_{\bullet}, L_{\mathrm{bol}}$, and $L_{\mathrm{bol}} / L_{\mathrm{Edd}}$ differences between the MMT and SDSS subsamples, and between point and extended sources. At all redshift ranges, the MMT identified quasars have a lower median $L_{\mathrm{bol}} / L_{\mathrm{Edd}}$ ratio than their SDSS counterparts, possibly related to the inclusion of extended sources in the MMT sample, as the mean $L_{\mathrm{bol}} / L_{\mathrm{Edd}}$ ratio is also lower for extended targets at all redshift.

At $z<1$, the extended sources show lower $L_{\text {bol }}(\sim 0.4$ dex $)$ and lower ER (by a factor of two) than the point sources (Figure 25). It is possible that the extended quasars reside in brighter or more massive host galaxies, and at a less active evolutionary phase with lower $L_{\mathrm{bol}} / L_{\mathrm{Edd}}$. Of the 12 targets with rather low ERs $\left(L_{\mathrm{bol}} / L_{\mathrm{Edd}}<0.01\right), 10$ are extended sources. Of the remaining 58 extended sources at $z<1,16$ have a $L_{\mathrm{bol}} / L_{\mathrm{Edd}}>0.1$ and 42 are at $0.01<L_{\mathrm{bol}} / L_{\mathrm{Edd}}<$ 0.1 . The $L_{\text {bol }} / L_{\text {Edd }}$ may be underestimated as quasars may contribute significantly in the rest-frame FIR as suggested by Kuraszkiewicz et al. (2003) and Dai et al. (2012). On the other hand, the ER may also be overestimated because of the possible host contribution to the $L_{\mathrm{bol}}$ at $z<1$; although the reddening correction of the spectra will counteract that effect. In the spectrum of at least a few MIR-selected SDSS sources with extended photometry, stellar absorption and sometimes a Balmer break are observed. For example, all six newly identified
SDSS quasars with extended morphology show signatures of the host galaxy (e.g., Figure 6) —all have Ca II H\&K absorption, and four also show the $G$-band in absorption.

At $1<z<3$, the MMT identified subsample has systematically lower $L_{\text {bol }}$ and $M$. than its SDSS counterparts (Figure 24, see also Table 10). The MMT sources extend the SDSS selection to fainter magnitudes (Figure 5), so at a given redshift they must either have lower $L_{\mathrm{bol}} / L_{\mathrm{Edd}}$ or smaller $M_{\text {. }}$. Kelly \& Shen (2013) suggested that the sub-Eddington boundary found for SDSS quasars was a magnitude-limit effect, and there was a large population of low $L_{\text {bol }} / L_{\text {Edd }}$ quasars down to $M_{\bullet} \sim 5 \times 10^{8} M_{\odot}\left(\log \left(M_{\bullet}\right)=8.7 M_{\odot}\right)$ and $L_{\mathrm{bol}} / L_{\mathrm{Edd}} \sim 0.07$ $\left(\log \left(L_{\text {bol }} / L_{\text {Edd }}\right)=-1.15\right)$. These do not appear in the MIRquasar population for $1<z<3$. Instead of a shift of the $M$. and $L_{\mathrm{bol}} / L_{\mathrm{Edd}}$ to smaller values, comparable mean and scatter of ERs and $M_{\bullet}$ are observed at $1<z<2$ and $2<z<3$ (Figure 25). At $1<z<3$, the point sources also scatter into the $<0.1 L_{\text {bol }} / L_{\text {edd }}$ regime. However, given the small numbers of extended objects at $z>1$-due to the resolution restrictions of the telescope-it is difficult to tell whether there is any systematic difference in the SMBH accretion rate between the extended and point-like quasars at earlier cosmic time.

\section{THE SPECTRAL CATALOG}

We have included all the measured properties from line fitting, and the derived properties in the online master catalogs. The catalogs are available in entirety in machine-readable form in the online journal. Objects are arranged in increasing RA order, 

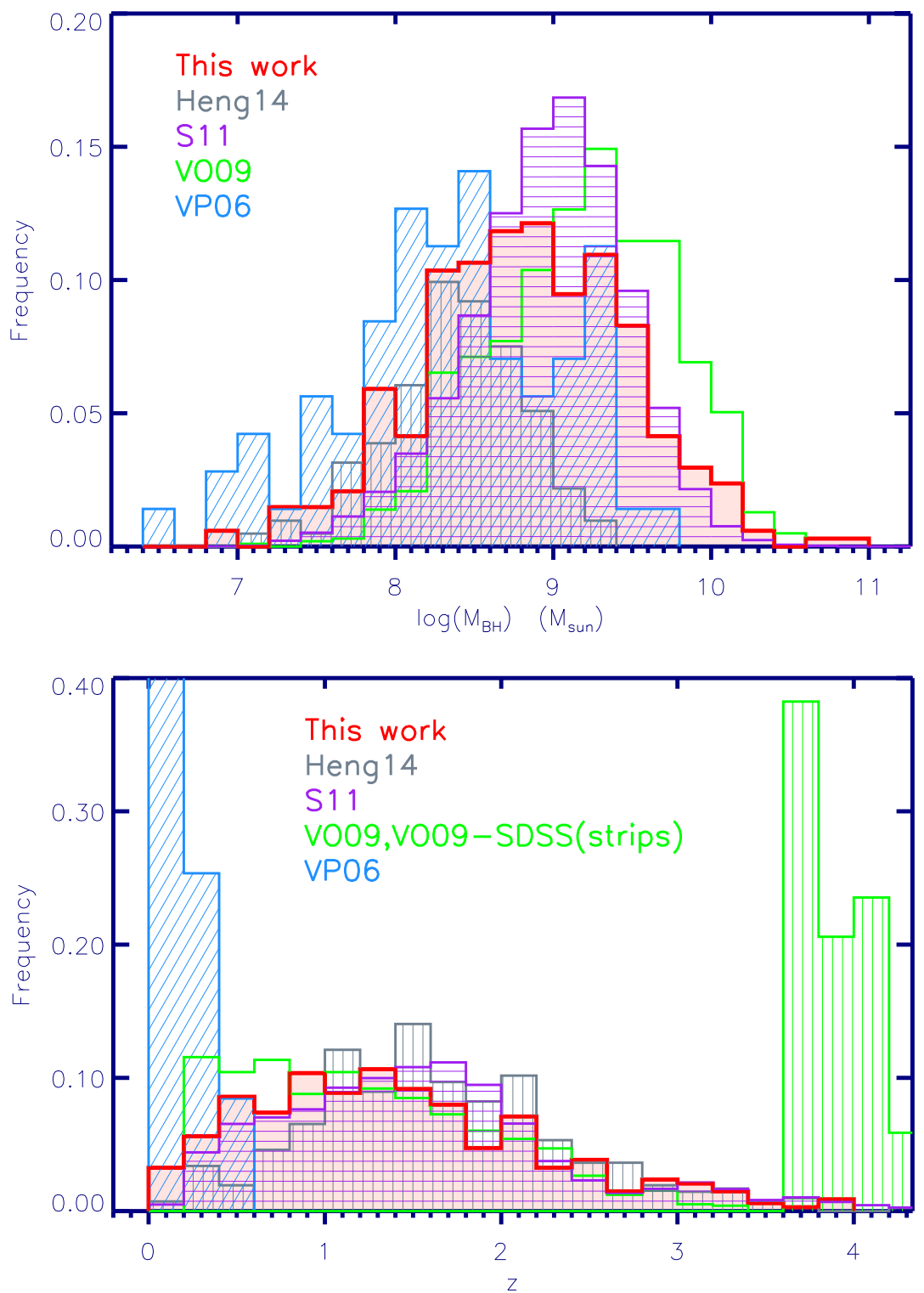

Figure 22. Top: the BH mass distribution of the relative frequency of the 391 MIR-selected quasar sample (red). Literature values from SDSS and other surveys are also plotted for comparison: gray, $413 \mathrm{X}$-ray-selected quasars (Hao et al. 2014), purple,105,783 SDSS-selected quasars (Shen et al. 2011, S11), blue, 1012 $z<5$ quasars, including 34 SDSS quasars at $3.5<z<5$ (Vestergaard \& Osmer 2009, VO09), green, 71z<0.3 quasars with RM info (Vestergaard \& Peterson 2006, VP06). Bottom: the redshift distribution of the MIR-selected quasars (red). Samples from the literature are color-coded in the same way as the top panel. The MIR-selected quasar sample overlaps with Hao14, S11, VO09-BQS, LBQS quasars in redshifts, and has a large overlap in $M_{\bullet}$ with the SDSS quasars. VP06 extends to the low mass end partly due to its relatively lower redshift from the RM constraint.

(A color version of this figure is available in the online journal.)

and the ID reflects the spectroscopic subsamples: MMT09, MMT05b, and SDSS. The same catalogs for the MMT05f faint objects are also provided for comparison. Table 11 lists all the basic parameters, including the object ID, position, redshift, SDSS, and MIPS $24 \mu \mathrm{m}$ photometries of the quasar sample; Table 12 lists the results, including flags, luminosities, SMBH mass, and ERs; Table 13 includes the fitting parameters, including continuum normalization and slope, iron template normalization and broadening, wavelength, S/N, FWHM, line area, and EW of each emission line. Unless otherwise stated, a null value is given if no measurements are available.

\section{DISCUSSION}

The catalog of MIR-selected quasars can be used to study the statistics of type 1 quasars and their physical properties.
We find that a significant and constant fraction (20\%) of MIR-selected quasars have extended optical photometry at $z<1.5$, indicating luminous host galaxies (Table 3). The MMT-recovered quasars include a small population of redder targets than the SDSS quasars (Figure 8). The MMT quasars share similar distributions with the SDSS quasars in all colors, including the fainter objects at $i \leqslant 20.2$ (Figures 10-12). The SDSS quasar algorithm is biased toward point sources at $i>19.1$ and is therefore missing quasars residing in extended hosts. Unresolved quasars comprise about $94 \%$ of all SDSS quasars. SDSS did not include extended objects in their target selection based on the assumption that the expected yield of quasars would be low. The MIR flux limit used in this sample is more inclusive and recovers the otherwise rejected extended sources. The extended population consists of $20 \%$ of the total MIR quasar population, and calls for a re-examination 


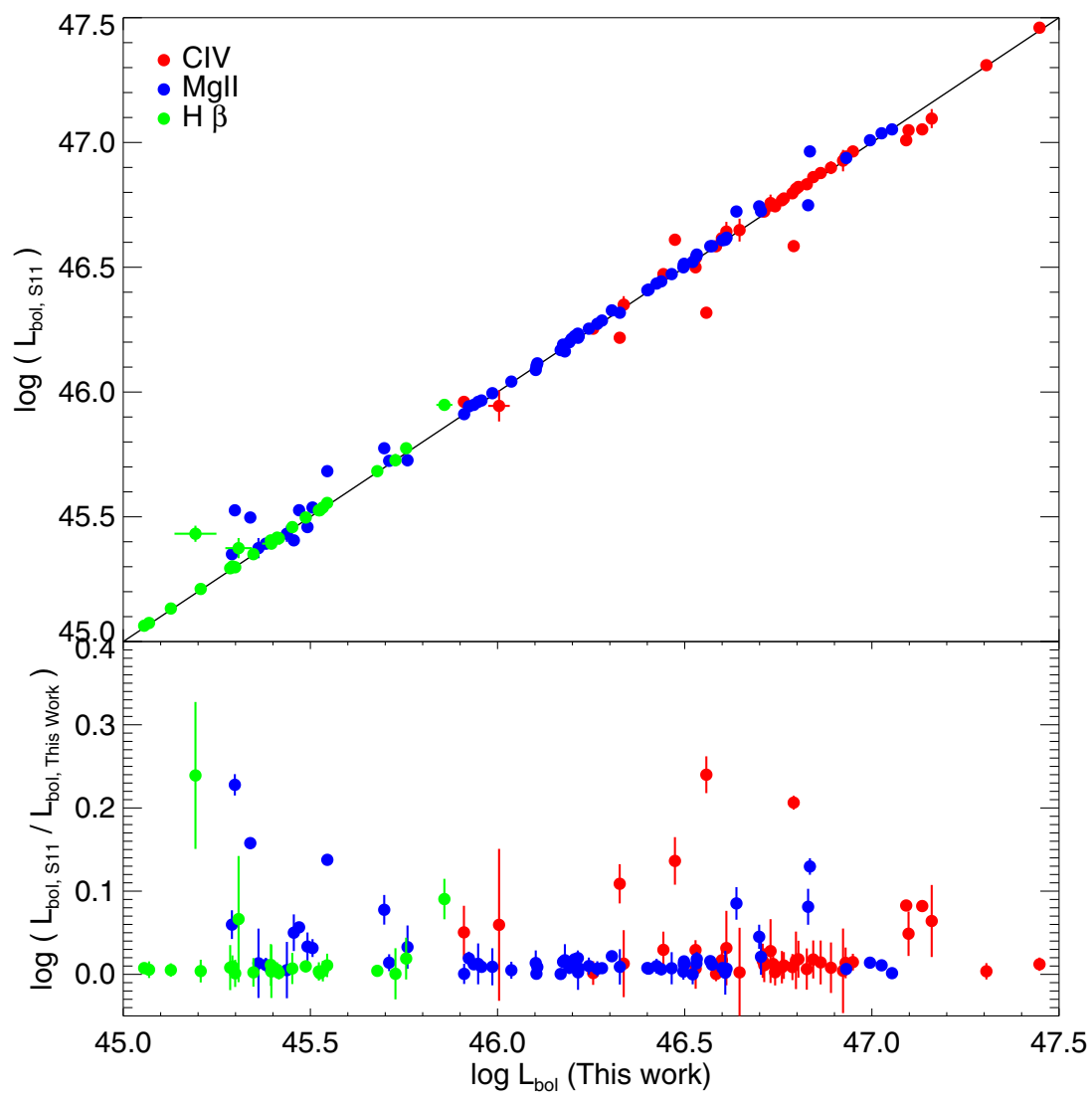

Figure 23. Bolometric luminosities ( $L_{\mathrm{bol}}$, top panel) and $L_{\mathrm{bol}}$ comparisons for the same targets between SDSS DR7 and this work (bottom, panel) as a function of $L_{\text {bol }}$. Only objects with a median line $\mathrm{S} / \mathrm{N}$ per pixel $>3$ are included. Targets with NALs/BALs are excluded. The two independently derived $L_{\text {bol }}$ are consistent with each other $(<3 \sigma)$ for the majority $(\sim 80 \%)$ of the MIR-selected SDSS subsample.

(A color version of this figure is available in the online journal.)

Table 10

Median SMBH Mass, Bolometric Luminosity, and Eddington Ratios of the MIR-selected Quasars

\begin{tabular}{|c|c|c|c|c|c|}
\hline Redshift & Subsample & No.obj & $\begin{array}{c}\log \left(M_{\bullet}\right) \\
\left(M_{\odot}\right)\end{array}$ & $\begin{array}{l}\log \left(L_{\text {bol }}\right) \\
\left(\mathrm{erg} \mathrm{s}^{-1}\right)\end{array}$ & $\log \left(L_{\text {bol }} / L_{\text {edd }}\right)$ \\
\hline \multirow[t]{3}{*}{$z<1$} & SDSS & 44 & $8.26 \pm 0.53$ & $45.21 \pm 0.40$ & $-1.07 \pm 0.53$ \\
\hline & MMT & 82 & $8.39 \pm 0.56$ & $45.01 \pm 0.46$ & $-1.33 \pm 0.55$ \\
\hline & Overall & 126 & $8.34 \pm 0.55$ & $45.06 \pm 0.44$ & $-1.24 \pm 0.55$ \\
\hline \multirow[t]{3}{*}{$1<z<2$} & SDSS & 55 & $9.05 \pm 0.47$ & $46.10 \pm 0.61$ & $-1.05 \pm 0.32$ \\
\hline & MMT & 126 & $8.85 \pm 0.44$ & $45.72 \pm 0.53$ & $-1.14 \pm 0.34$ \\
\hline & Overall & 181 & $8.91 \pm 0.45$ & $45.81 \pm 0.56$ & $-1.10 \pm 0.33$ \\
\hline \multirow[t]{3}{*}{$2<z<3$} & SDSS & 22 & $9.59 \pm 0.24$ & $46.80 \pm 0.36$ & $-0.98 \pm 0.32$ \\
\hline & MMT & 43 & $9.29 \pm 0.52$ & $46.27 \pm 0.44$ & $-1.15 \pm 0.38$ \\
\hline & Overall & 65 & $9.40 \pm 0.48$ & $46.37 \pm 0.50$ & $-1.05 \pm 0.37$ \\
\hline \multirow[t]{3}{*}{$z>3$} & SDSS & 17 & $9.92 \pm 0.47$ & $46.86 \pm 0.28$ & $-0.90 \pm 0.44$ \\
\hline & MMT & 2 & $10.78 \pm 1.27$ & $47.69 \pm 1.11$ & $-0.95 \pm 0.16$ \\
\hline & Overall & 19 & $9.92 \pm 0.54$ & $46.86 \pm 0.38$ & $-0.91 \pm 0.42$ \\
\hline Redshift & Type & No.obj & $\begin{array}{c}\log \left(M_{\bullet}\right) \\
\left(M_{\odot}\right)\end{array}$ & $\begin{array}{l}\log \left(L_{\text {bol }}\right) \\
\left(\operatorname{erg~s}^{-1}\right) \\
\end{array}$ & $\log \left(L_{\mathrm{bol}} / L_{\text {edd }}\right)$ \\
\hline \multirow[t]{2}{*}{$z<1$} & Point & 58 & $8.34 \pm 0.46$ & $45.29 \pm 0.41$ & $-1.07 \pm 0.46$ \\
\hline & Ext & 68 & $8.38 \pm 0.61$ & $44.93 \pm 0.42$ & $-1.34 \pm 0.59$ \\
\hline \multirow[t]{2}{*}{$1<z<2$} & Point & 172 & $8.91 \pm 0.45$ & $45.81 \pm 0.57$ & $-1.10 \pm 0.34$ \\
\hline & Ext & 96 & $8.90 \pm 0.34$ & $45.95 \pm 0.35$ & $-1.24 \pm 0.24$ \\
\hline \multirow[t]{2}{*}{$2<z<3$} & Point & 61 & $9.44 \pm 0.48$ & $46.40 \pm 0.49$ & $-1.05 \pm 0.38$ \\
\hline & Ext & 4 & $9.12 \pm 0.26$ & $45.94 \pm 0.49$ & $-1.25 \pm 0.24$ \\
\hline \multirow[t]{2}{*}{$z>3$} & Point & 19 & $9.92 \pm 0.54$ & $46.86 \pm 0.38$ & $-0.91 \pm 0.42$ \\
\hline & Ext & $\cdots$ & $\ldots$ & $\ldots$ & $\ldots$ \\
\hline
\end{tabular}




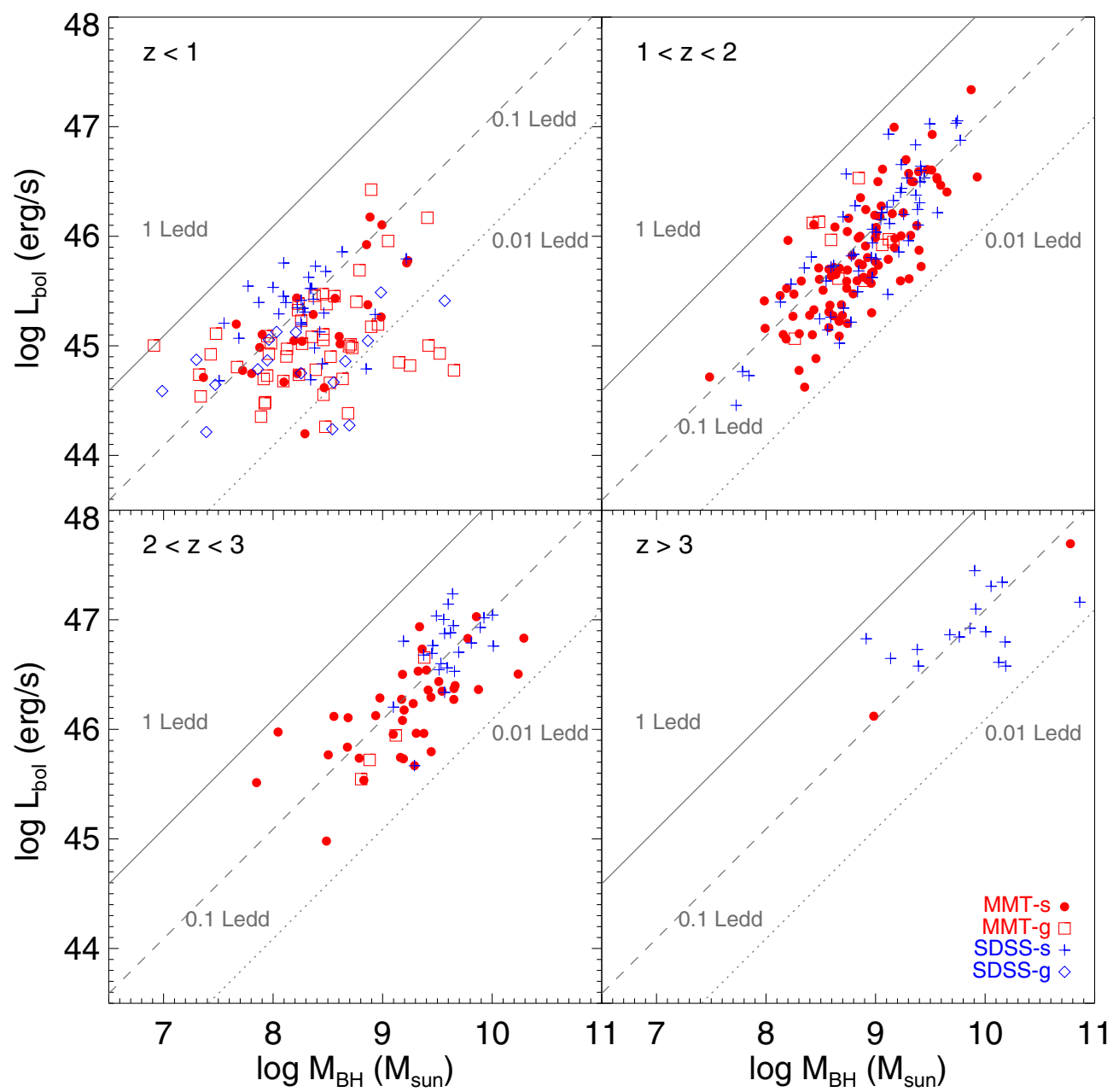

Figure 24. Bolometric luminosity $\left(L_{\text {bol }}\right)$ of MIR-selected quasars as a function of SMBH mass $\left(M_{\bullet}\right)$. The diagonal line marks the Eddington luminosity $\left(L_{\text {edd }}\right)$ of corresponding $M_{\bullet}$ at 1 (solid line), 0.1 (dashed line), and 0.01 (dotted line) $L_{\text {edd }}$. We separate the MMT (red) and SDSS (blue) subsamples by their morphologies, pluses and filled circles for the "star-like" point sources, and open diamonds and squares for the "galaxy-like" extended sources (see Section 2.7). The MMT quasars have lower $L_{\mathrm{bol}}$ and $M_{\bullet}$ than their SDSS counterparts at $z<3$.

(A color version of this figure is available in the online journal.)

Table 11

The MIR-selected Quasar Catalog 1. Properties

\begin{tabular}{lcl}
\hline \hline Column & Format & \\
\hline $1 \ldots$ & STRING & MMT designation of observation date-fiber number, \\
$\ldots$ & $\ldots$ & or SDSS DR7 designation of spectroscopic MJD + plate number + fiber number \\
$2 \ldots$ & DOUBLE & Right ascension in decimal degrees $(J 2000.0)$ \\
$3 \ldots$ & DOUBLE & Declination in decimal degrees $(J 2000.0)$ \\
$4 \ldots$ & DOUBLE & Redshift (see Section 2.5$)$ \\
$5 \ldots$ & DOUBLE & $24 \mu \mathrm{m}$ flux density $\left(S_{24}\right)$ from SWIRE photometry \\
$6 \ldots$ & DOUBLE & Uncertainty in $24 \mu \mathrm{m}$ flux density $\left(S_{24}{ }_{E} R\right.$ R $)$ from SWIRE photometry \\
$7-11$ & DOUBLE & SDSS photometry in AB magnitude $($ p.u, p.g, p.r, p.i, p.z) \\
$12-16$ & DOUBLE & Uncertainty in SDSS photometry (p.Err_u, p.Err_g, p.Err_r, p.Err_i, p.Err_z) \\
\hline
\end{tabular}

(Supplemental data (tar.gz) for this table are available in the online journal.)

and updated simulations for quasar distributions at all redshifts.

Although the SDSS algorithm completeness was simulated and found to be consistent with MIR color-selected quasar samples, e.g., Lacy et al. (2013), we discovered additional quasars using the flux-limited MIR-selection. At $i>19.1$, nine additional MIR quasars that meet the SDSS selection were recovered with the MMT spectroscopy, resulting in an updated SDSS completeness of $70 \%$. At $i<20.2$ and $z>2.9$, we only found one additional MIR quasar, which is consistent with the SDSS completeness of $90 \%$. This completeness difference arises from the different selection criteria, as both optical and MIR color selections restrict the sample to power-law like SEDs, whereas the MIR flux selection adopted here includes everything that meets the apparent magnitude requirement. At $z<3$ and $i>19.1$, the observed quasar number densities per square degree are higher than at the SDSS covered $i<19.1$ region. 


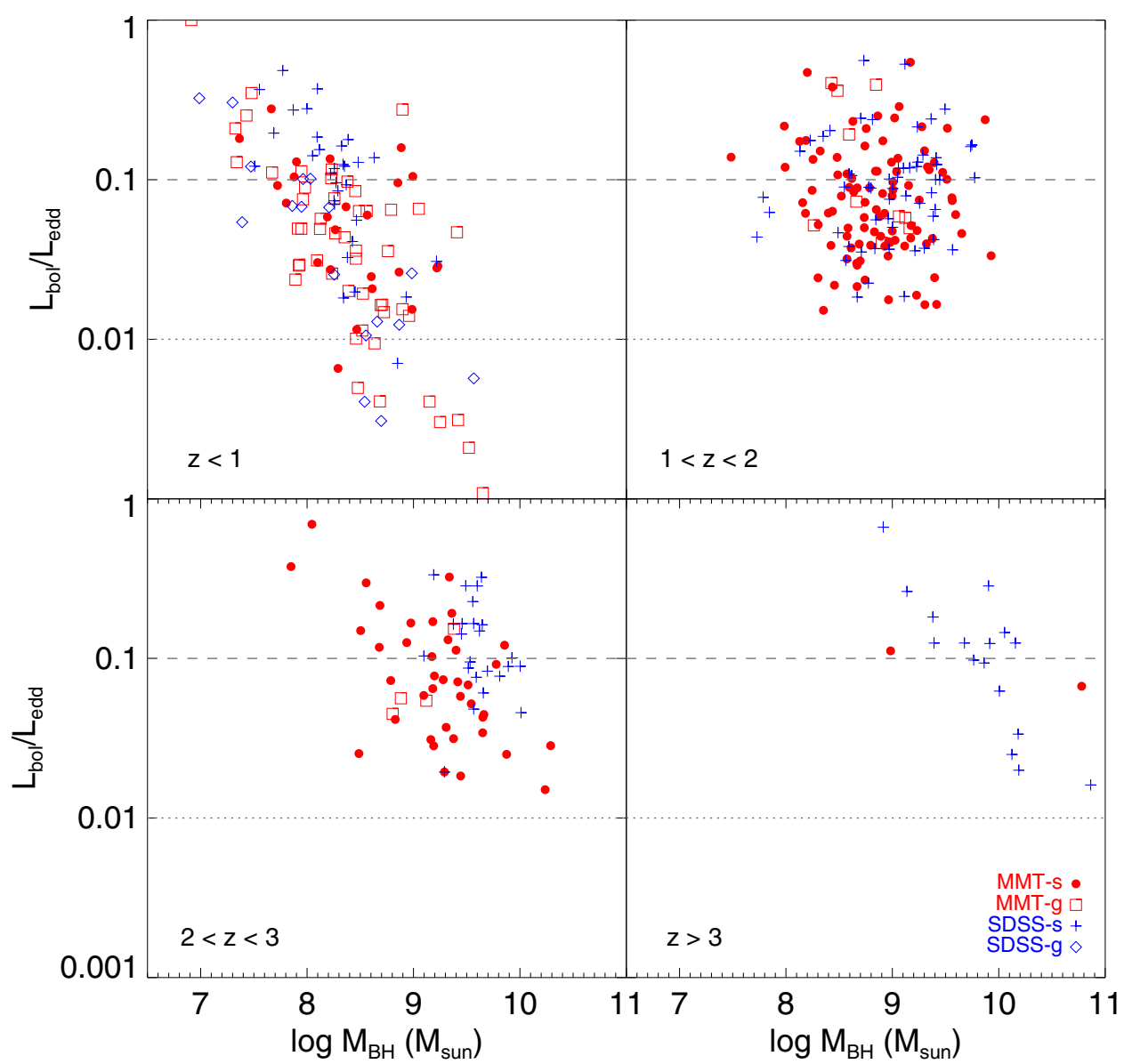

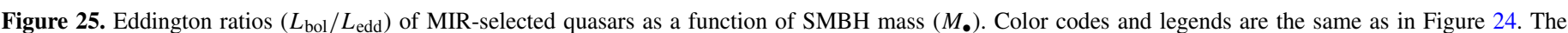

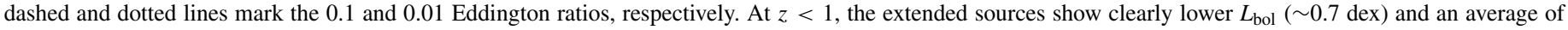

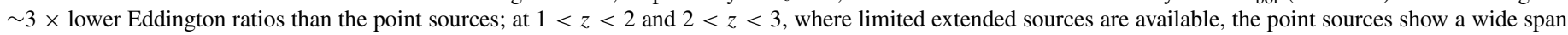
of Eddington ratios and scatter into the $L_{\text {bol }} / L_{\text {edd }}<0.1$ regime.

(A color version of this figure is available in the online journal.)

In Figure 7, the MIR-selected quasars show a redshift distribution peaking at $z \sim 1.4$, which is consistent with previous studies of the cosmic evolution of AGN number densities (Hasinger et al. 2005; Silverman et al. 2008). We see evidence of downsizing in the MIR-selected targets, with the most massive SMBHs appearing at earlier times, although the ER remains almost constant at $1<z<4$ with large scatters. Objects with low $L_{\text {bol }} / L_{\text {Edd }}<0.01$ are also observed at $z<1$.

Controversies exist as to whether CIV line-widths are attributed solely to gravity, or are affected by outflows or jets, and, as a result, whether the C IV emission derived masses are as reliable as $\mathrm{Mg}$ II and $\mathrm{H} \beta$ derived masses (VP06; Shen et al. 2008; Assef et al. 2011). This concern arises from the typically blueshifted CIV BEL peak compared to other quasars BELs (Gaskell 1982; Richards et al. 2002b, S11), the commonly observed BAL/NALs (Weymann et al. 1981; Wild et al. 2008) within the CIV emission line profiles, and the strong line asymmetries (Wilkes 1984; Richards et al. 2002b, See also Section 3). The blueshift of the C IV BEL peak relative Mg II is observed in $\sim 80 \%$ of the MIR-quasars whose spectra covers both CIV and Mg II BELs. In the MIR-selected quasar sample, there is no strong correlation between the C IV and Mg II FWHMs (Figure. 20, left). There is also no strong trend of decreasing ratios of $\log \left(M_{\mathrm{BH},(\mathrm{Mg} \text { II })} / M_{\mathrm{BH},(\mathrm{CIV})}\right)$ with increasing $\mathrm{C}$ IV-Mg II blueshifts, in contrast to the correlation reported in
S11 and Richards et al. (2011), although the scatter is large for both $M_{\bullet}$ ratios and $\mathrm{C}$ IV-Mg II blueshifts (Figure 27).

A non-virial C IV emission component can be used to explain the large scatter observed between C IV and other BEL derived M. (S11; Richards et al. 2011; Denney 2012). Denney (2012) found a "non-variable, largely core" emission component in the C IV BEL by comparing the SE spectra to the RM spectra. After removing this non-variable component, the $\mathrm{C}$ IV-derived $M_{\bullet}$ shows a better correlation with the $\mathrm{H} \beta$ derived $M_{\bullet}$. In this MIR-selected quasar sample, we found that the $M_{\bullet}$ derived from the dominant CIV FWHM shows a marginally better correlation with the $M_{\bullet}$ from Mg II BEL (slope coefficient $=$ $0.61 \pm 0.11)$ than that from the non-parametric C IV FWHM (slope coefficient $=0.42 \pm 0.07$; Figure 20, right) and has smaller scatter. If a non-parametric C IV FWHM is used instead, a sudden jump in the $M_{\bullet}$ distribution at $z \sim 1.6$ would appear, where the $M_{\bullet}$ starts to be derived from the CIV BELs. This sudden increase is not physical and supports our choice of the dominant CIV FWHM. In $70 \%$ of the CIV BEL with multiple Gaussians, the non-parametric CIV FWHMs are smaller than the dominant CIV FWHMs, due to contributions from narrower Gaussians that fit the line core (e.g., Figure 16). These narrower additional Gaussian components resemble the non-virial emission component found in Denney (2012, Figure 3 ). The marginally better correlation of dominant 


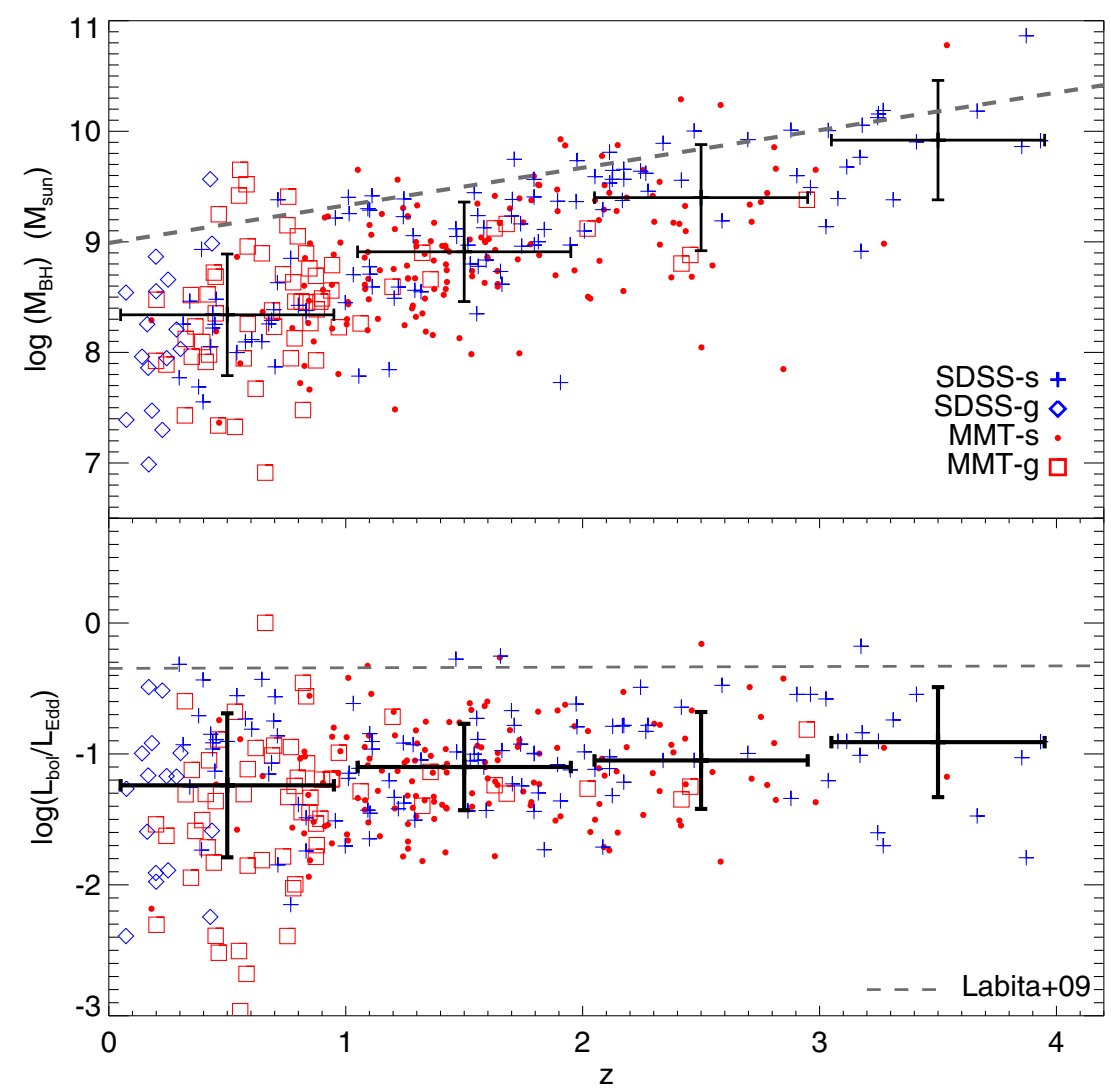

Figure 26. SMBH mass $\left(M_{\bullet}\right.$, top) and Eddington ratios $\left(L_{\mathrm{bol}} / L_{\mathrm{Edd}}\right.$, bottom) of MIR-selected quasars as a function of redshift. Color codes and legends are the same as in Figure 24. The dashed lines are the proposed maximum mass values $\left(M_{\bullet}(\max )=0.34 z+8.99\right)$ and Eddington ratios $\left(L_{\mathrm{bol}} / L_{\mathrm{Edd}}(\max )=0.005 z+0.45\right)$ from Labita et al. (2009). The mean and standard deviation in each redshift bin is marked by black pluses. We observe a downsizing effect in $M_{\bullet}$, but a more or less constant Eddington ratio across the cosmic time.

(A color version of this figure is available in the online journal.)

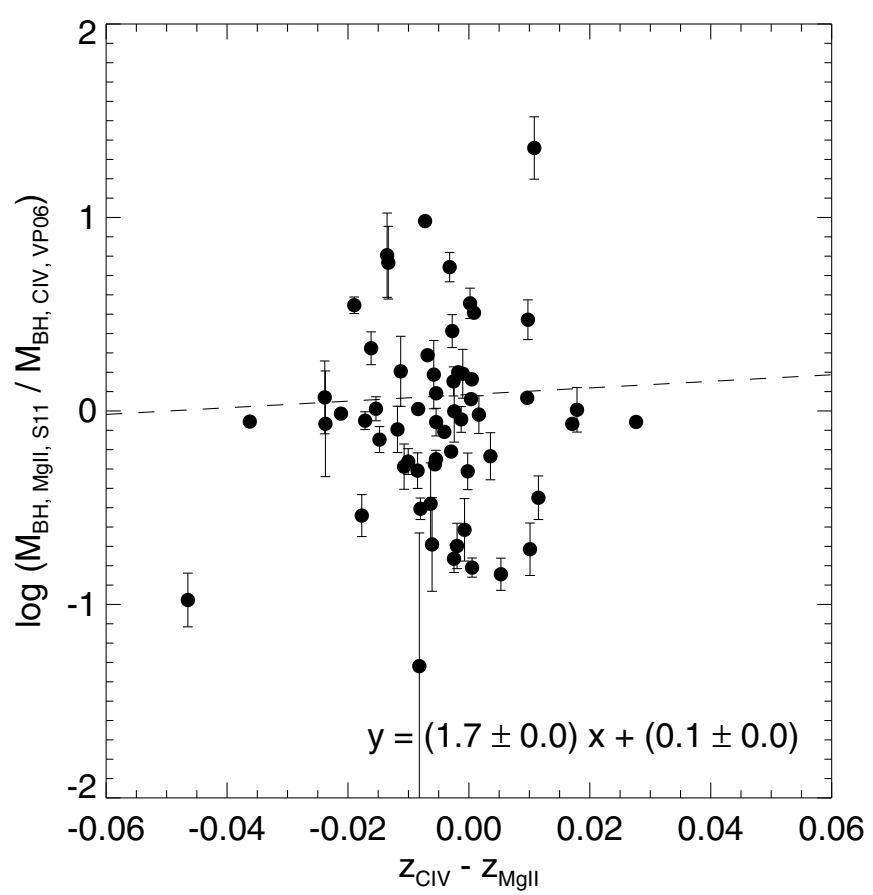

Figure 27. Comparison of the $\mathrm{C} I V-\mathrm{Mg}$ II redshift and $M_{\bullet}$ differences in the same objects covering both $\mathrm{C}$ IV and Mg II BELs. Blueshifted C IV corresponds to negative values. The $M_{\bullet}$ ratios indicate a loose to null correlation with the redshift difference $\left(z_{\mathrm{C}_{\text {IV }}}-z_{\mathrm{Mg} \text { II }}\right)$.
C IV $M_{\bullet}$ to $\mathrm{Mg}$ II derived $M_{\bullet}$ suggests contamination from non-

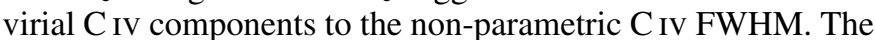
choice of dominant CIV FWHM instead of the conventional non-parametric FWHM for $M_{\bullet}$ estimates may provide a way to tackle this problem.

We find a high fraction of objects with absorption features in the MIR-selected sample. For C IV, $\sim 40 \%$ of the BELs quasars show absorption, NALs or BALs; and this fraction is $\sim 20 \%$ for $\mathrm{Mg}$ II objects. The fraction of BALs in MIR-selected quasars, is $17 \% \pm 3 \%$ in C IV, and $10 \% \pm 2 \%$ in $\mathrm{Mg}$ II. The C IV numbers agree with the overall fraction of $10 \%$ to $15 \%$ found for SDSS quasars (e.g., Trump et al. 2006; Knigge et al. 2008). The Mg II quasars show a $>3 \sigma$ higher fraction of BALs than the $1.31 \%$ in Trump et al. (2006). A velocity offset $\left(v_{\text {off }}\right)$ of $\left|v_{\text {off }}\right| \leqslant 3000 \mathrm{~km} \mathrm{~s}^{-1}$ between the NAL/BALs and the system redshift is commonly used to define the associated NALs (e.g., Wild et al. 2008; Shen \& Ménard 2012). The boundary between the NAL and BAL widths also differs from paper to paper. Wild et al. (2008) used an upper boundary of $700 \mathrm{~km} \mathrm{~s}^{-1}$ for associated NALs, and Shen \& Ménard (2012) used $500 \mathrm{~km} \mathrm{~s}^{-1}$. Here we adopt the $700 \mathrm{~km} \mathrm{~s}^{-1}$ limit and find the fraction of NALs to be $17 \%$ (27 objects) for C IV and 13\% (40 objects) for $\mathrm{Mg}$ II. These fractions are $\sim 3 \sigma$ higher for both $\mathrm{C}$ IV and $\mathrm{Mg}$ II NALs than in the SDSS color-selected quasars (Wild et al. 2008). They are consistent with the SDSS quasars within errors for high velocity ( $>3000 \mathrm{~km} \mathrm{~s}^{-1}$ ) narrow absorbers (Vestergaard 2003; Wild et al. 2008). About $25 \%$ and $20 \%$ of the C IV and Mg II 
Table 12

The MIR-selected Quasar Catalog 2. Results

\begin{tabular}{|c|c|c|}
\hline Column & Format & Description \\
\hline 1. & STRING & MMT designation of observation date-fiber number \\
\hline$\ldots$ & $\ldots$ & or SDSS DR7 designation of spectroscopic MJD + plate number + fiber number \\
\hline $2 \ldots$ & INTEGER & extended source flag (p.type), “3” for extended object, “6” for point source \\
\hline $3 \ldots$ & INTEGER & absorption flag, "1" for sources with absorption, "0" for targets without absorption \\
\hline $4 \ldots$ & INTEGER & faint object flag, "1" for sources with $S_{24}<400 \mu \mathrm{Jy}$-the MMT05f subsample \\
\hline $5 \ldots$ & DOUBLE & Fiducial Virial SMBH mass $\log M_{\bullet}$ in $M_{\odot}($ Section 4$)$ \\
\hline $6 \ldots$ & DOUBLE & Measurement uncertainty of the fiducial $\log M_{\bullet}$ in percentage \\
\hline $7 \ldots$ & DOUBLE & Bolometric luminosity $\log L_{\text {bol }}$ in erg s ${ }^{-1}$ \\
\hline $8 \ldots$ & DOUBLE & Uncertainty in $\log L_{\text {bol }}$ in percentage \\
\hline $9 \ldots$ & DOUBLE & Eddington ratio $\left(L_{\mathrm{bol}} / L_{\mathrm{edd}}\right)$ based on the fiducial $M_{\bullet}$ \\
\hline $10 \ldots$ & DOUBLE & Virial SMBH mass based on dominant $\mathrm{C}$ IV, $\log M_{\mathrm{BH}, \mathrm{C} \text { Iv VP06 in } M_{\odot}}$ \\
\hline $11 \ldots$ & DOUBLE & Measurement uncertainty in dominant $\mathrm{C}$ IV, $\log M_{\mathrm{BH}, \mathrm{C} \text { IV VP06 }}$ in percentage \\
\hline $12 \ldots$ & DOUBLE & 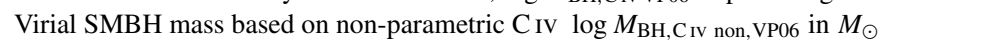 \\
\hline $13 \ldots$ & DOUBLE & Measurement uncertainty in non-parametric $\mathrm{C}_{\mathrm{IV}}, \log M_{\mathrm{BH}, \mathrm{C} \text { IV non,VP06 in percentage }}$ \\
\hline $14 \ldots$ & DOUBLE & Virial SMBH mass based on dominant $\mathrm{Mg}$ II, $\log M_{\mathrm{BH}, \mathrm{Mg} \text { II } \mathrm{MD} 04}$ in $M_{\odot}$ \\
\hline $15 \ldots$ & DOUBLE & Measurement uncertainty in dominant $\mathrm{Mg}$ II, $\log M_{\mathrm{BH}, \mathrm{Mg} \text { II MD04 in percentage }}$ \\
\hline $16 \ldots$ & DOUBLE & 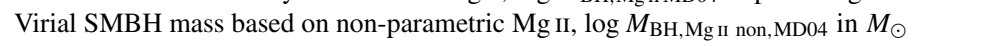 \\
\hline $17 \ldots$ & DOUBLE & Measurement uncertainty in non-parametric $\mathrm{Mg}$ II, $\log M_{\mathrm{BH}, \mathrm{Mg} \text { II non,MD04 }}$ in percentage \\
\hline $18 \ldots$ & DOUBLE & Virial SMBH mass based on dominant $\mathrm{Mg}$ II, $\log M_{\mathrm{BH}, \mathrm{Mg}_{\text {II }} \text { VO09 in } M_{\odot}}$ \\
\hline $19 \ldots$ & DOUBLE & 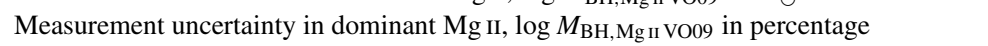 \\
\hline $20 \ldots$ & DOUBLE & 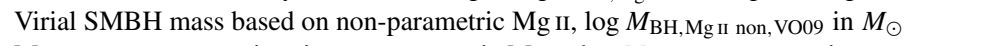 \\
\hline $21 \ldots$ & DOUBLE & Measurement uncertainty in non-parametric $\mathrm{Mg}$ II, $\log M_{\mathrm{BH}, \mathrm{Mg} \text { II non, VO09 in percentage }}$ \\
\hline $22 \ldots$ & DOUBLE & Virial SMBH mass based on dominant $\mathrm{Mg}$ II, $\log M_{\mathrm{BH}, \mathrm{Mg}_{\text {II S}} 11}$ in $M_{\odot}$ \\
\hline $23 \ldots$ & DOUBLE & Measurement uncertainty in dominant $\mathrm{Mg}$ II, $\log M_{\mathrm{BH}, \mathrm{Mg} \text { II }} \mathrm{S}_{11}$ in percentage \\
\hline $24 \ldots$ & DOUBLE & Virial SMBH mass based on non-parametric $\mathrm{Mg}_{\mathrm{II}}, \log M_{\mathrm{BH}, \mathrm{Mg} \text { II non,S11 }}$ in $M_{\odot}$ \\
\hline $25 \ldots$ & DOUBLE & Measurement uncertainty in non-parametric $\mathrm{Mg}$ II, $\log M_{\mathrm{BH}, \mathrm{Mg} \text { II non,S11 }}$ in percentage \\
\hline $26 \ldots$ & DOUBLE & Virial SMBH mass based on dominant $\mathrm{H} \beta, \log M_{\mathrm{BH}, \mathrm{H} \beta, \mathrm{VP} 06}$ in $M_{\odot}$ \\
\hline $27 \ldots$ & DOUBLE & Measurement uncertainty in dominant $\mathrm{H} \beta, \log M_{\mathrm{BH}, \mathrm{H} \beta, \mathrm{VP} 06}$ in percentage \\
\hline $28 \ldots$ & DOUBLE & Virial SMBH mass based on dominant $\mathrm{H} \beta, \log M_{\mathrm{BH}, \mathrm{H} \beta, \mathrm{VO} 09}$ in $M_{\odot}$ \\
\hline $29 \ldots$ & DOUBLE & Measurement uncertainty in dominant $\mathrm{H} \beta, \log M_{\mathrm{BH}, \mathrm{H} \beta, \mathrm{VO} 09}$ in percentage \\
\hline $30 \ldots$ & DOUBLE & Monochromatic line luminosity at $1350 \AA \log L_{1350}$ in $\mathrm{erg} \mathrm{s}^{-1}$ \\
\hline $31 \ldots$ & DOUBLE & Uncertainty in $\log L_{1350}$ in percentage \\
\hline $32 \ldots$ & DOUBLE & Monochromatic line luminosity at $3000 \AA \log L_{3000}$ in $\mathrm{erg} \mathrm{s}^{-1}$ \\
\hline $33 \ldots$ & DOUBLE & Uncertainty in $\log L_{3000}$ in percentage \\
\hline $34 \ldots$ & DOUBLE & Monochromatic line luminosity at $5100 \AA \log L_{5100}$ in $\mathrm{erg} \mathrm{s}^{-1}$ \\
\hline $35 \ldots$ & DOUBLE & Uncertainty in $\log L_{5100}$ in percentage \\
\hline $36 \ldots$ & DOUBLE & Bolometric luminosity $\log L_{\text {bol_C }}$ IV in $\mathrm{erg} \mathrm{s}^{-1}\left(0.580925+\log L_{1350}\right)$ \\
\hline $37 \ldots$ & DOUBLE & Uncertainty in $\log L_{\text {bol_C IV in percentage }}$ \\
\hline $38 \ldots$ & DOUBLE & Bolometric luminosity $\log L_{\text {bol }}-\mathrm{Mg}$ II in $\operatorname{erg~s}^{-1}\left(0.711807+\log L_{3000}\right)$ \\
\hline $39 \ldots$ & DOUBLE & Uncertainty in $\log L_{\text {bol_}} \mathrm{Mg}$ II in percentage \\
\hline $40 \ldots$ & DOUBLE & Bolometric luminosity $\log L_{\text {bol }} \mathrm{H} \beta$ in $\operatorname{erg~s}^{-1}\left(0.96661+\log L_{5100}\right)$ \\
\hline $41 \ldots$ & DOUBLE & Uncertainty in $\log L_{\text {bol }} \mathrm{H} \beta$ in percentage \\
\hline $42 \ldots$ & DOUBLE & 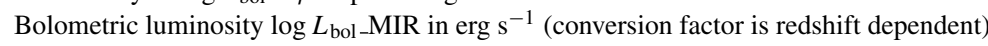 \\
\hline $43 \ldots$ & DOUBLE & Uncertainty in $\log L_{\text {bol }}$ MIR in percentage \\
\hline
\end{tabular}

Notes. Notes: Unless otherwise stated, null value is given if not measured, and -1 for its associated error.

(Supplemental data (tar.gz) for this table are available in the online journal.)

absorbers show absorptions redshifted from the emission line peak, indicating possible inflows toward the SMBH in the BLR. We will present the subsample of quasars with redshifted C IV absorption and explore the possibilities in a forthcoming paper (Y. S. Dai et al. in preparation).

\section{SUMMARY}

We construct a catalog of MIR-selected quasars in the Lockman Hole-SWIRE field and present their SMBH mass and Eddington ratios in this paper. This BEL, type 1 quasar sample is MIPS $24 \mu \mathrm{m}$ selected and optically identified in three spectroscopic surveys: MMT09, MMT05b, and SDSS. In the catalog we compiled their photometries, continuum and emission line properties, and luminosities and virial SMBH mass $\left(M_{\bullet}\right)$ derived from the spectral measurements.
We find a significant population of quasars with extended photometric morphologies. A constant fraction of $20 \%$ of extended objects are observed in the MIR-selected quasars across the magnitude ranges. We then compare and estimate the completeness of the SDSS quasar selection algorithm to be $\sim 70 \%$ at $i<19.1$, about $20 \%$ lower than the reported $90 \%$ completeness (R02). At $19.1<i<20.2$ and $z>3$, our result is consistent with the reported SDSS completeness. At $z<3$, SDSS only covered the $i<19.1$ region. In this redshift range, we observe a significantly higher quasar number density at $19.1<i<20.2\left(\sim 24 \mathrm{deg}^{-2}\right)$ than at $i<19.1\left(\sim 10 \mathrm{deg}^{-2}\right)$. The number density at $i>20.2$ is even higher, reaching $\sim 45 \mathrm{deg}^{-2}$. The MIR selection used here efficiently extends the magnitude limit of the quasar population to the low- $z$ sources. Compared to color selections, the MIR selection recovers a 
Table 13

The MIR-selected Quasar Catalog -3. Parameters

\begin{tabular}{|c|c|c|}
\hline Column & Format & Description \\
\hline $1 \ldots$ & STRING & MMT designation of observation date-fiber number, \\
\hline$\ldots$ & $\ldots$ & or SDSS DR7 designation of spectroscopic MJD + plate number + fiber number \\
\hline $2 \ldots$ & DOUBLE & Power-law normalization for C IV continuum fit at $3000 \AA$ \\
\hline $3 \ldots$ & DOUBLE & Uncertainty in Power-law normalization \\
\hline $4 \ldots$ & DOUBLE & Power-law slope $\alpha_{\mathrm{C}_{\text {IV }}}$ for the continuum fit \\
\hline $5 \ldots$ & DOUBLE & Uncertainty in $\alpha_{\mathrm{C} \text { IV }}$ \\
\hline $6 \ldots$ & DOUBLE & Central wavelength of the dominant C IV component \\
\hline $7 \ldots$ & DOUBLE & Central wavelength of the second C IV component \\
\hline $8 \ldots$ & DOUBLE & Central wavelength of the third C IV component \\
\hline $9 \ldots$ & DOUBLE & Central wavelength of the non-parametric $\mathrm{C}$ IV component \\
\hline $10 \ldots$ & DOUBLE & Line dispersion $\left(\sigma_{l}\right)$ of the dominant $\mathrm{C}$ IV component in $\mathrm{km} \mathrm{s}^{-1}$ \\
\hline $11 \ldots$ & DOUBLE & Uncertainty in $\sigma_{l}$ of the dominant C IV component in $\mathrm{km} \mathrm{s}^{-1}$ \\
\hline $12 \ldots$ & DOUBLE & Full-width-half-maximum (FWHM) of the dominant C IV in $\mathrm{km} \mathrm{s}^{-1}$ \\
\hline $13 \ldots$ & DOUBLE & Uncertainty in the dominant $\mathrm{C}$ IV FWHM in $\mathrm{km} \mathrm{s}^{-1}$ \\
\hline $14 \ldots$ & DOUBLE & Integrated line area of the dominant $C_{I V}$ \\
\hline $15 \ldots$ & DOUBLE & Uncertainty in the integrated line area of the dominant $\mathrm{C}$ IV \\
\hline $16 \ldots$ & DOUBLE & Restframe equivalent width (EW) of the dominant C IV ( \\
\hline $17 \ldots$ & DOUBLE & Uncertainty in EW of the dominant C IV \\
\hline $18 \ldots$ & DOUBLE & Line dispersion $\left(\sigma_{l}\right)$ of the secondary $\mathrm{C}$ IV component in $\mathrm{km} \mathrm{s}^{-1}$ \\
\hline $19 \ldots$ & DOUBLE & Uncertainty in $\sigma_{l}$ of the secondary C IV component in $\mathrm{km} \mathrm{s}^{-1}$ \\
\hline & DOUBLE & Full-width-half-maximum (FWHM) of the secondary $\mathrm{C}_{\mathrm{IV}}$ in $\mathrm{km} \mathrm{s}^{-1}$ \\
\hline $21 \ldots$ & DOUBLE & Uncertainty in the secondary C IV FWHM in $\mathrm{km} \mathrm{s}^{-1}$ \\
\hline $22 \ldots$ & DOUBLE & Integrated line area of the secondary C IV \\
\hline $23 \ldots$ & DOUBLE & Uncertainty in the integrated line area of the secondary C IV \\
\hline $24 \ldots$ & DOUBLE & Restframe equivalent width (EW) of the secondary C IV ( $\AA$ ) \\
\hline $25 \ldots$ & DOUBLE & Uncertainty in EW of the secondary C IV \\
\hline $26 \ldots$ & DOUBLE & Line dispersion $\left(\sigma_{l}\right)$ of the third C IV component in $\mathrm{km} \mathrm{s}^{-1}$ \\
\hline $27 \ldots$ & DOUBLE & Uncertainty in $\sigma_{l}$ of the third C IV component in $\mathrm{km} \mathrm{s}^{-1}$ \\
\hline $28 \ldots$ & DOUBLE & Full-width-half-maximum (FWHM) of the third C IV in $\mathrm{km} \mathrm{s}^{-1}$ \\
\hline $29 \ldots$ & DOUBLE & Uncertainty in the third C IV FWHM in $\mathrm{km} \mathrm{s}^{-1}$ \\
\hline $30 \ldots$ & DOUBLE & Integrated line area of the third $\mathrm{C}$ IV \\
\hline & DOUBLE & Uncertainty in the integrated line area of the third C IV \\
\hline $32 \ldots$ & DOUBLE & Restframe equivalent width (EW) of the third C IV ( $\AA$ ) \\
\hline $33 \ldots$ & DOUBLE & Uncertainty in EW of the third C IV \\
\hline $34 \ldots$ & DOUBLE & Full-width-half-maximum (FWHM) of the non-parametric $\mathrm{C}$ IV in $\mathrm{km} \mathrm{s}^{-1}$ \\
\hline $35 \ldots$ & DOUBLE & Uncertainty in the non-parametric C IV FWHM in $\mathrm{km} \mathrm{s}^{-1}$ \\
\hline $36 \ldots$ & DOUBLE & Integrated line area of the non-parametric C IV \\
\hline $36 \ldots$ & DOUBLE & Uncertainty in the integrated line area of the non-parametric C IV \\
\hline $38 \ldots$ & DOUBLE & 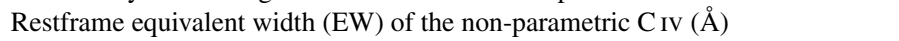 \\
\hline $39 \ldots$ & DOUBLE & Uncertainty in EW of the non-parametric C IV \\
\hline $40 \ldots$ & DOUBLE & Reduced $\chi^{2}$ for the C IV continuum fit \\
\hline $41 \ldots$ & DOUBLE & Reduced $\chi^{2}$ for the $\mathrm{C}$ iv emission line fit \\
\hline $42 \ldots$ & SHORT & Status code for the C IV continuum fit (See IDL program "mpfitfun.pro") \\
\hline $43 \ldots$ & SHORT & Status code for the $\mathrm{C}$ IV emission line fit \\
\hline $44 \ldots$ & SHORT & Number of good pixels for the $C$ IV emission line fitting region $(1500-1600 \AA)$ \\
\hline $45 \ldots$ & DOUBLE & Median S/N per pixel for the $\mathrm{C}$ IV emission line fitting region \\
\hline $46 \ldots$ & DOUBLE & Power-law normalization for $\mathrm{Mg}$ II continuum fit at $3000 \AA$ \\
\hline $47 \ldots$ & DOUBLE & Uncertainty in Power-law normalization \\
\hline $48 \ldots$ & DOUBLE & Power-law slope $\alpha_{\mathrm{Mg} \text { II }}$ for the continuum fit \\
\hline $49 \ldots$ & DOUBLE & Uncertainty in $\alpha_{\mathrm{Mg} \text { II }}$ \\
\hline $50 \ldots$ & DOUBLE & Normalization of the Fe II template \\
\hline $51 \ldots$ & DOUBLE & Uncertainty in Fe II normalization \\
\hline $52 \ldots$ & DOUBLE & FWHM of the Fe II component for Mg II continuum fit \\
\hline $53 \ldots$ & DOUBLE & Uncertainty in $\mathrm{FWHM}_{\mathrm{Fe}}$ \\
\hline $54 \ldots$ & DOUBLE & Central wavelength of the dominant $\mathrm{Mg}$ II component \\
\hline $55 \ldots$ & DOUBLE & Central wavelength of the second $\mathrm{Mg}$ II component \\
\hline $56 \ldots$ & DOUBLE & Central wavelength of the third $\mathrm{Mg}$ II component \\
\hline $57 \ldots$ & DOUBLE & Central wavelength of the narrow Mg II component \\
\hline $58 \ldots$ & DOUBLE & Central wavelength of the non-parametric $\mathrm{Mg}$ II component \\
\hline $59 \ldots$ & DOUBLE & Line dispersion $\left(\sigma_{l}\right)$ of the dominant $\mathrm{Mg}$ II component in $\mathrm{km} \mathrm{s}^{-1}$ \\
\hline $60 \ldots$ & DOUBLE & Uncertainty in $\sigma_{l}$ of the dominant $\mathrm{Mg}$ II component in $\mathrm{km} \mathrm{s}^{-1}$ \\
\hline $61 \ldots$ & DOUBLE & Full-width-half-maximum (FWHM) of the dominant $\mathrm{Mg}$ II in $\mathrm{km} \mathrm{s}^{-1}$ \\
\hline $62 \ldots$ & DOUBLE & Uncertainty in the dominant $\mathrm{Mg}$ II FWHM in $\mathrm{km} \mathrm{s}^{-1}$ \\
\hline $63 \ldots$ & DOUBLE & Integrated line area of the dominant $\mathrm{Mg}$ II \\
\hline $64 \ldots$ & DOUBLE & Uncertainty in the integrated line area of the dominant $\mathrm{Mg}$ II \\
\hline $65 \ldots$ & DOUBLE & Restframe equivalent width (EW) of the dominant $\mathrm{Mg}_{\text {II }}(\AA)$ \\
\hline
\end{tabular}


Table 13

(Continued)

\begin{tabular}{|c|c|c|}
\hline Column & Format & Description \\
\hline $66 \ldots$ & DOUBLE & Uncertainty in EW of the dominant $\mathrm{Mg}$ II \\
\hline $67 \ldots$ & DOUBLE & Line dispersion $\left(\sigma_{l}\right)$ of the secondary $\mathrm{Mg}$ II component in $\mathrm{km} \mathrm{s}^{-1}$ \\
\hline $68 \ldots$ & DOUBLE & Uncertainty in $\sigma_{l}$ of the secondary $\mathrm{Mg}$ II component in $\mathrm{km} \mathrm{s}^{-1}$ \\
\hline $69 \ldots$ & DOUBLE & Full-width-half-maximum (FWHM) of the secondary $\mathrm{Mg}$ II in $\mathrm{km} \mathrm{s}^{-1}$ \\
\hline $70 \ldots$ & DOUBLE & Uncertainty in the secondary $\mathrm{Mg}$ II FWHM in $\mathrm{km} \mathrm{s}^{-1}$ \\
\hline $71 \ldots$ & DOUBLE & Integrated line area of the secondary $\mathrm{Mg}$ II \\
\hline $72 \ldots$ & DOUBLE & Uncertainty in the integrated line area of the secondary $\mathrm{Mg}$ II \\
\hline $73 \ldots$ & DOUBLE & Restframe equivalent width (EW) of the secondary $\mathrm{Mg}_{\text {II }}(\AA)$ \\
\hline $74 \ldots$ & DOUBLE & Uncertainty in EW of the secondary $\mathrm{Mg}_{\mathrm{II}}$ \\
\hline $75 \ldots$ & DOUBLE & Line dispersion $\left(\sigma_{l}\right)$ of the third $\mathrm{Mg}_{\text {II }}$ component in $\mathrm{km} \mathrm{s}^{-1}$ \\
\hline $76 \ldots$ & DOUBLE & Uncertainty in $\sigma_{l}$ of the third $\mathrm{Mg}$ II component in $\mathrm{km} \mathrm{s}^{-1}$ \\
\hline $77 \ldots$ & DOUBLE & Full-width-half-maximum (FWHM) of the third $\mathrm{Mg}_{\mathrm{II}}$ in $\mathrm{km} \mathrm{s}^{-1}$ \\
\hline $78 \ldots$ & DOUBLE & Uncertainty in the third $\mathrm{Mg}_{\text {II }} \mathrm{FWHM}$ in $\mathrm{km} \mathrm{s}^{-1}$ \\
\hline $79 \ldots$ & DOUBLE & Integrated line area of the third $\mathrm{Mg}$ II \\
\hline $80 \ldots$ & DOUBLE & Uncertainty in the integrated line area of the third $\mathrm{Mg}$ II \\
\hline $81 \ldots$ & DOUBLE & Restframe equivalent width (EW) of the third $\mathrm{Mg}$ II $(\AA)$ \\
\hline $82 \ldots$ & DOUBLE & Uncertainty in EW of the third $\mathrm{Mg}$ II \\
\hline $83 \ldots$ & DOUBLE & Full-width-half-maximum (FWHM) of the non-parametric $\mathrm{Mg}_{\text {II }}$ in $\mathrm{km} \mathrm{s}^{-1}$ \\
\hline $84 \ldots$ & DOUBLE & Uncertainty in the non-parametric $\mathrm{Mg}$ II FWHM in $\mathrm{km} \mathrm{s}^{-1}$ \\
\hline $85 \ldots$ & DOUBLE & Integrated line area of the non-parametric $\mathrm{Mg}_{\mathrm{II}}$ \\
\hline $86 \ldots$ & DOUBLE & Uncertainty in the integrated line area of the non-parametric $\mathrm{Mg}_{\mathrm{II}}$ \\
\hline $87 \ldots$ & DOUBLE & Restframe equivalent width (EW) of the non-parametric $\mathrm{Mg}_{\text {II }}(\AA)$ \\
\hline $88 \ldots$ & DOUBLE & Uncertainty in EW of the non-parametric $\mathrm{Mg}_{\mathrm{II}}$ \\
\hline $89 \ldots$ & DOUBLE & Line dispersion $\left(\sigma_{l}\right)$ of the narrow $\mathrm{Mg}$ II component in $\mathrm{km} \mathrm{s}^{-1}$ \\
\hline $90 \ldots$ & DOUBLE & Uncertainty in $\sigma_{l}$ of the narrow $\mathrm{Mg}$ II component in $\mathrm{km} \mathrm{s}^{-1}$ \\
\hline $91 \ldots$ & DOUBLE & Full-width-half-maximum (FWHM) of the narrow $\mathrm{Mg}_{\text {II }}$ in $\mathrm{km} \mathrm{s}^{-1}$ \\
\hline $92 \ldots$ & DOUBLE & Uncertainty in the narrow Mg II FWHM in $\mathrm{km} \mathrm{s}^{-1}$ \\
\hline $93 \ldots$ & DOUBLE & Integrated line area of the narrow $\mathrm{Mg}$ II \\
\hline $94 \ldots$ & DOUBLE & Uncertainty in the integrated line area of the narrow Mg II \\
\hline $95 \ldots$ & DOUBLE & Restframe equivalent width (EW) of the narrow $\mathrm{Mg}_{\text {II }}(\AA)$ \\
\hline $96 \ldots$ & DOUBLE & Uncertainty in EW of the narrow $\mathrm{Mg}$ II \\
\hline $97 \ldots$ & DOUBLE & Reduced $\chi^{2}$ for the $\mathrm{Mg}$ II continuum fit \\
\hline $98 \ldots$ & DOUBLE & Reduced $\chi^{2}$ for the Mg II emission line fit \\
\hline $99 \ldots$ & SHORT & Status code for the Mg II continuum fit (See IDL program "mpfitfun.pro") \\
\hline $100 \ldots$ & SHORT & Status code for the $\mathrm{Mg}$ II emission line fit \\
\hline $101 \ldots$ & SHORT & Number of good pixels for the $\mathrm{Mg}_{\text {II }}$ emission line fitting region $(2700-2900 \AA)$ \\
\hline $102 \ldots$ & DOUBLE & Median $\mathrm{S} / \mathrm{N}$ per pixel for the $\mathrm{Mg}$ II emission line fitting region \\
\hline $103 \ldots$ & DOUBLE & Power-law normalization for $\mathrm{H} \beta$ continuum fit at $3000 \AA$ \\
\hline $104 \ldots$ & DOUBLE & Uncertainty in Power-law normalization \\
\hline $105 \ldots$ & DOUBLE & Power-law slope $\alpha_{\mathrm{H} \beta}$ for the continuum fit \\
\hline $106 \ldots$ & DOUBLE & Uncertainty in $\alpha_{\mathrm{H} \beta}$ \\
\hline $107 \ldots$ & DOUBLE & Normalization of the $\mathrm{Fe}$ II $+\left[\mathrm{O}_{\text {III }}\right]$ template \\
\hline $108 \ldots$ & DOUBLE & Uncertainty in Fe II + [O III] normalization \\
\hline $109 \ldots$ & DOUBLE & FWHM of the $\mathrm{Fe} \mathrm{II}+[\mathrm{O} \mathrm{III}]$ component for $\mathrm{H} \beta$ continuum fit \\
\hline $110 \ldots$ & DOUBLE & Uncertainty in $\mathrm{FWHM}_{\mathrm{Fe}}$ \\
\hline $111 \ldots$ & DOUBLE & Central wavelength of the dominant $\mathrm{H} \beta$ component \\
\hline $112 \ldots$ & DOUBLE & Central wavelength of the second $\mathrm{H} \beta$ component \\
\hline $113 \ldots$ & DOUBLE & Central wavelength of the third $\mathrm{H} \beta$ component \\
\hline $114 \ldots$ & DOUBLE & Central wavelength of the narrow $\mathrm{H} \beta$ component \\
\hline $115 \ldots$ & DOUBLE & Central wavelength of the $[\mathrm{O}$ III] $\lambda 4959$ component \\
\hline $116 \ldots$ & DOUBLE & Central wavelength of the $[\mathrm{O}$ III $] \lambda 5007$ component \\
\hline $117 \ldots$ & DOUBLE & Line dispersion $\left(\sigma_{l}\right)$ of the dominant $\mathrm{H} \beta$ component in $\mathrm{km} \mathrm{s}^{-1}$ \\
\hline $118 \ldots$ & DOUBLE & Uncertainty in $\sigma_{l}$ of the dominant $\mathrm{H} \beta$ component in $\mathrm{km} \mathrm{s}^{-1}$ \\
\hline $119 \ldots$ & DOUBLE & Full-width-half-maximum (FWHM) of the dominant $\mathrm{H} \beta$ in $\mathrm{km} \mathrm{s}^{-1}$ \\
\hline $120 \ldots$ & DOUBLE & Uncertainty in the dominant $\mathrm{H} \beta$ FWHM in $\mathrm{km} \mathrm{s}^{-1}$ \\
\hline $121 \ldots$ & DOUBLE & Integrated line area of the dominant $\mathrm{H} \beta$ \\
\hline $122 \ldots$ & DOUBLE & Uncertainty in the integrated line area of the dominant $\mathrm{H} \beta$ \\
\hline $123 \ldots$ & DOUBLE & Restframe equivalent width (EW) of the dominant $\mathrm{H} \beta(\AA)$ \\
\hline $124 \ldots$ & DOUBLE & Uncertainty in EW of the dominant $\mathrm{H} \beta$ \\
\hline $125 \ldots$ & DOUBLE & Line dispersion $\left(\sigma_{l}\right)$ of the secondary $\mathrm{H} \beta$ component in $\mathrm{km} \mathrm{s}^{-1}$ \\
\hline $126 \ldots$ & DOUBLE & Uncertainty in $\sigma_{l}$ of the secondary $\mathrm{H} \beta$ component in $\mathrm{km} \mathrm{s}^{-1}$ \\
\hline $127 \ldots$ & DOUBLE & Full-width-half-maximum (FWHM) of the secondary $\mathrm{H} \beta$ in $\mathrm{km} \mathrm{s}^{-1}$ \\
\hline $128 \ldots$ & DOUBLE & Uncertainty in the secondary $\mathrm{H} \beta$ FWHM in $\mathrm{km} \mathrm{s}^{-1}$ \\
\hline $129 \ldots$ & DOUBLE & Integrated line area of the secondary $\mathrm{H} \beta$ \\
\hline $130 \ldots$ & DOUBLE & Uncertainty in the integrated line area of the secondary $\mathrm{H} \beta$ \\
\hline $131 \ldots$ & DOUBLE & Restframe equivalent width (EW) of the secondary $\mathrm{H} \beta(\AA)$ \\
\hline
\end{tabular}


Table 13

(Continued)

\begin{tabular}{|c|c|c|}
\hline Column & Format & Description \\
\hline $132 \ldots$ & DOUBLE & Uncertainty in $\mathrm{EW}$ of the secondary $\mathrm{H} \beta$ \\
\hline $133 \ldots$ & DOUBLE & Line dispersion $\left(\sigma_{l}\right)$ of the third $\mathrm{H} \beta$ component in $\mathrm{km} \mathrm{s}^{-1}$ \\
\hline $134 \ldots$ & DOUBLE & Uncertainty in $\sigma_{l}$ of the third $\mathrm{H} \beta$ component in $\mathrm{km} \mathrm{s}^{-1}$ \\
\hline $135 \ldots$ & DOUBLE & Full-width-half-maximum (FWHM) of the third $\mathrm{H} \beta$ in $\mathrm{km} \mathrm{s}^{-1}$ \\
\hline $136 \ldots$ & DOUBLE & Uncertainty in the third $\mathrm{H} \beta$ FWHM in $\mathrm{km} \mathrm{s}^{-1}$ \\
\hline $137 \ldots$ & DOUBLE & Integrated line area of the third $\mathrm{H} \beta$ \\
\hline $138 \ldots$ & DOUBLE & Uncertainty in the integrated line area of the third $\mathrm{H} \beta$ \\
\hline $139 \ldots$ & DOUBLE & Restframe equivalent width (EW) of the third $\mathrm{H} \beta(\AA)$ \\
\hline $140 \ldots$ & DOUBLE & Uncertainty in EW of the third $\mathrm{H} \beta$ \\
\hline $141 \ldots$ & DOUBLE & Line dispersion $\left(\sigma_{l}\right)$ of the narrow $\mathrm{H} \beta$ component in $\mathrm{km} \mathrm{s}^{-1}$ \\
\hline $142 \ldots$ & DOUBLE & Uncertainty in $\sigma_{l}$ of the narrow $\mathrm{H} \beta$ component in $\mathrm{km} \mathrm{s}^{-1}$ \\
\hline $143 \ldots$ & DOUBLE & Full-width-half-maximum (FWHM) of the narrow $\mathrm{H} \beta$ in $\mathrm{km} \mathrm{s}^{-1}$ \\
\hline $144 \ldots$ & DOUBLE & Uncertainty in the narrow $\mathrm{H} \beta$ FWHM in $\mathrm{km} \mathrm{s}^{-1}$ \\
\hline $145 \ldots$ & DOUBLE & Integrated line area of the narrow $\mathrm{H} \beta$ \\
\hline $146 \ldots$ & DOUBLE & Uncertainty in the integrated line area of the narrow $\mathrm{H} \beta$ \\
\hline $147 \ldots$ & DOUBLE & Restframe equivalent width (EW) of the narrow $\mathrm{H} \beta(\AA)$ \\
\hline $148 \ldots$ & DOUBLE & Uncertainty in $\mathrm{EW}$ of the narrow $\mathrm{H} \beta$ \\
\hline $149 \ldots$ & DOUBLE & Line dispersion $\left(\sigma_{l}\right)$ of the $[\mathrm{O}$ III $] \lambda 4959$ component in $\mathrm{km} \mathrm{s}^{-1}$ \\
\hline $150 \ldots$ & DOUBLE & Uncertainty in $\sigma_{l}$ of the [O $\left.\mathrm{III}\right] \lambda 4959$ component in $\mathrm{km} \mathrm{s}^{-1}$ \\
\hline $151 \ldots$ & DOUBLE & 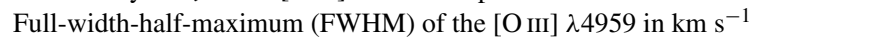 \\
\hline $152 \ldots$ & DOUBLE & Uncertainty in the $\left[\mathrm{O}_{\mathrm{III}}\right] \lambda 4959 \mathrm{FWHM}$ in $\mathrm{km} \mathrm{s}^{-1}$ \\
\hline $153 \ldots$ & DOUBLE & Integrated line area of the $\left[\mathrm{O}_{\text {III }}\right] \lambda 4959$ \\
\hline $154 \ldots$ & DOUBLE & Uncertainty in the integrated line area of the $[\mathrm{O}$ III $] \lambda 4959$ \\
\hline $155 \ldots$ & DOUBLE & Restframe equivalent width (EW) of the $[\mathrm{O}$ III] $\lambda 4959(\AA)$ \\
\hline $156 \ldots$ & DOUBLE & Uncertainty in EW of the $[\mathrm{O}$ III] $\lambda 4959$ \\
\hline $157 \ldots$ & DOUBLE & Line dispersion $\left(\sigma_{l}\right)$ of the $[\mathrm{O}$ III $] \lambda 5007$ component in $\mathrm{km} \mathrm{s}^{-1}$ \\
\hline $158 \ldots$ & DOUBLE & Uncertainty in $\sigma_{l}$ of the $\left[\mathrm{O}\right.$ III] $\lambda 5007$ component in $\mathrm{km} \mathrm{s}^{-1}$ \\
\hline $159 \ldots$ & DOUBLE & Full-width-half-maximum (FWHM) of the $[\mathrm{O}$ III $] \lambda 5007$ in $\mathrm{km} \mathrm{s}^{-1}$ \\
\hline $160 \ldots$ & DOUBLE & Uncertainty in the $[\mathrm{O}$ III $] \lambda 5007 \mathrm{FWHM}$ in $\mathrm{km} \mathrm{s}^{-1}$ \\
\hline $161 \ldots$ & DOUBLE & Integrated line area of the $\left[\mathrm{O}_{\mathrm{III}}\right] \lambda 5007$ \\
\hline $162 \ldots$ & DOUBLE & Uncertainty in the integrated line area of the $[\mathrm{O}$ III $] \lambda 5007$ \\
\hline $163 \ldots$ & DOUBLE & Restframe equivalent width (EW) of the $[\mathrm{O}$ III $] \lambda 5007(\AA)$ \\
\hline $164 \ldots$ & DOUBLE & Uncertainty in EW of the $[\mathrm{O}$ III $] \lambda 5007$ \\
\hline $165 \ldots$ & DOUBLE & Ratio of $([\mathrm{O}$ III $] \lambda 5007 /[\mathrm{O}$ III $] \lambda 4959)$ \\
\hline $166 \ldots$ & DOUBLE & Reduced $\chi^{2}$ for the $\mathrm{H} \beta$ continuum fit \\
\hline $167 \ldots$ & DOUBLE & Reduced $\chi^{2}$ for the $\mathrm{H} \beta$ emission line fit \\
\hline $168 \ldots$ & SHORT & Status code for the $\mathrm{H} \beta$ continuum fit (See IDL program "mpfitfun.pro") \\
\hline $169 \ldots$ & SHORT & Status code for the $\mathrm{H} \beta$ emission line fit \\
\hline $170 \ldots$ & SHORT & Number of good pixels for the $\mathrm{H} \beta$ emission line fitting region $(4700-5100 \AA)$ \\
\hline $171 \ldots$ & DOUBLE & Median $\mathrm{S} / \mathrm{N}$ per pixel for the $\mathrm{H} \beta$ emission line fitting region \\
\hline
\end{tabular}

Notes. Notes: (1) Unless otherwise stated, null value is given if not measured, and -1 for its associated error.

(Supplemental data (tar.gz) for this table are available in the online journal.)

high fraction of extended objects, and provides a more complete sample to study the total quasar population.

We measured the line-width and calculated the virial SMBH mass $\left(M_{\bullet}\right)$, bolometric luminosity $\left(L_{\mathrm{bol}}\right)$, and the ERs $\left(L_{\text {bol }} / L_{\text {edd }}\right)$ for the MIR-selected quasars. The consistency between the $M_{\bullet}$ estimated by the $\mathrm{C}$ IV $\mathrm{Mg}$ II and $\mathrm{H} \beta$ emission lines is also tested. We found a better correlation between $\mathrm{C}$ IV and $\mathrm{Mg}$ II derived $M_{\bullet}$ using the dominant $\mathrm{C}$ IV FWHM instead of the conventional non-parametric C IV FWHM, indicating a contribution from non-virial component to the latter.

The $\log M_{\bullet}\left(M_{\odot}\right)$ derived from emission line-width has an average error of $\sim 0.3$ dex, with a distribution from 7 to 11 , peaking at $\log M_{\bullet}\left(M_{\odot}\right)=8.8$. The MMT identified quasars supplement the SDSS quasars at lower $M_{\bullet}$, lower $L_{\mathrm{bol}}$, and in the SDSS exclusion zones. A systematic offset in Eddington ratios is found between extended and point sources at $z<1$, indicating a less active AGN phase for the extended objects. Similar large scatter of $L_{\text {bol }} / L_{\text {Edd }}$ from 0.01 to 1 is observed at $1<z<3$. The $M_{\bullet}$ for MIR-selected quasars shows a strong trend of downsizing, but the Eddington ratio remains relatively independent of redshift.

We also find a high fraction of quasars with absorption features in this MIR-selected sample, which will be presented in a forthcoming paper.

The complete catalogs are now publicly available online along with the MMT-Hectospec spectra. A similar $24 \mu$ m flux-limited redshift survey by the authors is underway in XMM-LSS, First Look Survey, and the Extended Groth Strip fields.

Y.S.D. acknowledges support from the Smithsonian Astrophysical Observatory (SAO) through the SAO Predoctoral Fellowship. We thank the anonymous referee for suggestions that led to the improvement of the manuscript. We thank Yue Shen for sharing the spectral measurement code, and Richard Cool for sharing and supporting of the HSRED reduction code.

Observations reported here were obtained at the MMT Observatory, a joint facility of the Smithsonian Institution and the University of Arizona. This work is also based partly on 
observations made with the Spitzer Space Telescope, operated by the Jet Propulsion Laboratory, California Institute of Technology under a contract with NASA. Funding for the SDSS and SDSS-II has been provided by the Alfred P. Sloan Foundation, the Participating Institutions, the National Science Foundation, the U.S. Department of Energy, the National Aeronautics and Space Administration, the Japanese Monbukagakusho, the Max Planck Society, and the Higher Education Funding Council for England. The SDSS Web site is http://www.sdss.org/.

Facilities: MMT, Spitzer, Sloan

\section{REFERENCES}

Abazajian, K. N., Adelman-McCarthy, J. K., Agüeros, M. A., et al. 2009, ApJS, 182,543

Anderson, S. F., Voges, W., Margon, B., et al. 2003, AJ, 126, 2209

Antonucci, R. 1993, ARA\&A, 31, 473

Assef, R. J., Denney, K. D., Kochanek, C. S., et al. 2011, ApJ, 742, 93

Bentz, M. C., Denney, K. D., Grier, C. J., et al. 2013, ApJ, 767, 149

Bentz, M. C., Peterson, B. M., Netzer, H., Pogge, R. W., \& Vestergaard, M. 2009, ApJ, 697, 160

Bentz, M. C., Peterson, B. M., Pogge, R. W., Vestergaard, M., \& Onken, C. A. 2006, ApJ, 644, 133

Bournaud, F., Chapon, D., Teyssier, R., et al. 2011, ApJ, 730, 4

Boroson, T. A., \& Green, R. F. 1992, ApJS, 80, 109

Caputi, K. I., Lagache, G., Yan, L., et al. 2007, ApJ, 660, 97

Collin, S., Kawaguchi, T., Peterson, B. M., \& Vestergaard, M. 2006, A\&A, 456,75

Cool, R. J., Eisenstein, D. J., Fan, X., et al. 2008, ApJ, 682, 919

Dai, Y. S., Bergeron, J., Elvis, M., et al. 2012, ApJ, 753, 33

Dekel, A., Birnboim, Y., Engel, G., et al. 2009, Natur, 457, 451

Denney, K. D. 2012, ApJ, 759, 44

Denney, K. D., Pogge, R. W., Assef, R. J., et al. 2013, ApJ, 775, 60

Di Matteo, T., Khandai, N., DeGraf, C., et al. 2012, ApJL, 745, L29

Dole, H., Rieke, G. H., Lagache, G., et al. 2004, ApJS, 154, 93

Donley, J. L., Koekemoer, A. M., Brusa, M., et al. 2012, ApJ, 748, 142

Elvis, M. 2000, ApJ, 545, 63

Elvis, M., Wilkes, B. J., McDowell, J. C., et al. 1994, ApJS, 95, 1

Fabricant, D., Fata, R., Roll, J., et al. 2005, PASP, 117, 1411

Fabricant, D. G., Kurtz, M. J., Geller, M. J., et al. 2008, PASP, 120, 1222

Falcke, H., Körding, E., \& Markoff, S. 2004, A\&A, 414, 895

Fazio, G. G., Hora, J. L., Allen, L. E., et al. 2004, ApJS, 154, 10

Ferrarese, L., \& Merritt, D. 2000, ApJL, 539, L9

Fine, S., Croom, S. M., Bland-Hawthorn, J., et al. 2010, MNRAS, 409, 591

Fukugita, M., Ichikawa, T., Gunn, J. E., et al. 1996, AJ, 111, 1748

Gaskell, C. M. 1982, ApJ, 263, 79

Gebhardt, K., Bender, R., Bower, G., et al. 2000, ApJL, 539, L13

Glikman, E., Gregg, M. D., Lacy, M., et al. 2004, ApJ, 607, 60

Glikman, E., Helfand, D. J., White, R. L., et al. 2007, ApJ, 667, 673

Glikman, E., Urrutia, T., Lacy, M., et al. 2012, ApJ, 757, 51

Goulding, A. D., Forman, W. R., Hickox, R. C., et al. 2014, ApJ, 783, 40

Hasinger, G., Miyaji, T., \& Schmidt, M. 2005, A\&A, 441, 417

Hao, H., Elvis, M., Civano, F., et al. 2014, MNRAS, 438, 1288

Hopkins, P. F., Hernquist, L., Cox, T. J., et al. 2006, ApJS, 163, 1

Jahnke, K., \& Macciò, A. V. 2011, ApJ, 734, 92

Juneau, S., Dickinson, M., Bournaud, F., et al. 2013, ApJ, 764, 176

Kaspi, S., Smith, P. S., Netzer, H., et al. 2000, ApJ, 533, 631

Kauffmann, G., Heckman, T. M., Tremonti, C., et al. 2003, MNRAS, 346, 1055

Kelly, B. C., \& Shen, Y. 2013, ApJ, 764, 45

Knigge, C., Scaringi, S., Goad, M. R., \& Cottis, C. E. 2008, MNRAS, 386, 1426

Kollmeier, J. A., Onken, C. A., Kochanek, C. S., et al. 2006, ApJ, 648, 128

Kormendy, J., \& Ho, L. C. 2013, ARA\&A, 51, 511

Kormendy, J., \& Richstone, D. 1995, ARA\&A, 33, 581

Kuraszkiewicz, J. K., Wilkes, B. J., Hooper, E. J., et al. 2003, ApJ, 590, 128

Kurucz, R. L. 1993, Kurucz CD-ROM13 (Cambridge, MA: Smithsonian Astrophysical Observatory)

Labita, M., Decarli, R., Treves, A., \& Falomo, R. 2009, MNRAS, 396, 1537

Lacy, M., Gregg, M., Becker, R. H., et al. 2002, AJ, 123, 2925

Lacy, M., Ridgway, S. E., Gates, E. L., et al. 2013, ApJS, 208, 24

Lacy, M., Storrie-Lombardi, L. J., Sajina, A., et al. 2004, ApJS, 154, 166

Laor, A., Bahcall, J. N., Jannuzi, B. T., et al. 1994, ApJ, 420, 110

Lilly, S. J., Carollo, C. M., Pipino, A., Renzini, A., \& Peng, Y. 2013, ApJ, 772,119
Lonsdale, C. J., Smith, H. E., Rowan-Robinson, M., et al. 2003, PASP 115,897

Marconi, A., \& Hunt, L. K. 2003, ApJL, 589, L21

Marziani, P., Sulentic, J. W., Dultzin-Hacyan, D., Calvani, M., \& Moles, M. 1996, ApJS, 104, 37

McLure, R. J., \& Dunlop, J. S. 2004, MNRAS, 352, 1390 (MD04)

McLure, R. J., \& Dunlop, J. S. 2002, MNRAS, 331, 795

Mink, D. J., Wyatt, W. F., Caldwell, N., et al. 2007, in ASP Conf. Ser. 376, Astronomical Data Analysis Software and Systems XVI, ed. R. A. Shaw, F. Hill, \& D. J. Bell (San Francisco, CA: ASP), 249

Netzer, H., Lutz, D., Schweitzer, M., et al. 2007, ApJ, 666, 806

Newman, J. A., Cooper, M. C., Davis, M., et al. 2013, ApJS, 208, 5

Ogle, P., Whysong, D., \& Antonucci, R. 2006, ApJ, 647, 161

Oke, J. B., \& Gunn, J. E. 1983, ApJ, 266, 713

Papovich, C., Cool, R., Eisenstein, D., et al. 2006, AJ, 132, 231

Park, D., Woo, J.-H., Denney, K. D., \& Shin, J. 2013, ApJ, 770, 87

Peng, C. Y. 2007, ApJ, 671, 1098

Peterson, B. M., Ferrarese, L., Gilbert, K. M., et al. 2004, ApJ, 613, 682

Pilbratt, G. L., Riedinger, J. R., Passvogel, T., et al. 2010, A\&A, 518, L1

Polletta, M. D. C., Wilkes, B. J., Siana, B., et al. 2006, ApJ, 642, 673

Press, W. H., Teukolsky, S. A., Vetterling, W. T., \& Flannery, B. P. 1992, Numerical Recipes in Fortran 77: The Art of Scientific Computing (2nd ed.; Cambridge: Cambridge Univ. Press)

Richards, G. T., Deo, R. P., Lacy, M., et al. 2009, AJ, 137, 3884

Richards, G. T., Fan, X., Newberg, H. J., et al. 2002a, AJ, 123, 2945

Richards, G. T., Hall, P. B., Vanden Berk, D. E., et al. 2003, AJ, 126, 1131

Richards, G. T., Kruczek, N. E., Gallagher, S. C., et al. 2011, AJ, 141, 167

Richards, G. T., Lacy, M., Storrie-Lombardi, L. J., et al. 2006a, ApJS, 166, 470

Richards, G. T., Strauss, M. A., Fan, X., et al. 2006b, AJ, 131, 2766

Richards, G. T., Vanden Berk, D. E., Reichard, T. A., et al. 2002b, AJ, 124, 1

Rieke, G. H., Young, E. T., Engelbracht, C. W., et al. 2004, ApJS, 154, 25

Sajina, A., Lacy, M., \& Scott, D. 2005, ApJ, 621, 256

Salvato, M., Hasinger, G., Ilbert, O., et al. 2009, ApJ, 690, 1250

Sanders, D. B., Soifer, B. T., Elias, J. H., et al. 1988, ApJ, 325, 74

Schlegel, D. J., Finkbeiner, D. P., \& Davis, M. 1998, ApJ, 500, 525

Schmidt, M., \& Green, R. F. 1983, ApJ, 269, 352

Schneider, D. P., Hall, P. B., Richards, G. T., et al. 2007, AJ, 134, 102

Schneider, D. P., Richards, G. T., Hall, P. B., et al. 2010, AJ, 139, 2360

Serjeant, S., Bertoldi, F., Blain, A. W., et al. 2010, A\&A, 518, L7

Shen, Y., Greene, J. E., Strauss, M. A., Richards, G. T., \& Schneider, D. P. 2008, ApJ, 680, 169

Shen, Y., \& Ménard, B. 2012, ApJ, 748, 131

Shen, Y., Richards, G. T., Strauss, M. A., et al. 2011, ApJS, 194, 45 (S11)

Shi, Y., Rieke, G. H., Ogle, P., Jiang, L., \& Diamond-Stanic, A. M. 2009, ApJ, 703, 1107

Shupe, D. L., Rowan-Robinson, M., Lonsdale, C. J., et al. 2008, AJ, 135, 1050 Silverman, J. D., Green, P. J., Barkhouse, W. A., et al. 2008, ApJ, 679, 118

Smith, J. A., Tucker, D. L., Kent, S., et al. 2002, AJ, 123, 2121

Steinhardt, C. L., \& Elvis, M. 2010, MNRAS, 402, 2637

Stern, D., Assef, R. J., Benford, D. J., et al. 2012, ApJ, 753, 30

Stern, D., Eisenhardt, P., Gorjian, V., et al. 2005, ApJ, 631, 163

Stoughton, C., Lupton, R. H., Bernardi, M., et al. 2002, AJ, 123, 485

Sturm, E., Rupke, D., Contursi, A., et al. 2006, ApJL, 653, L13

Sulentic, J. W., Bachev, R., Marziani, P., Negrete, C. A., \& Dultzin, D. 2007, ApJ, 666,757

Tremaine, S., Gebhardt, K., Bender, R., et al. 2002, ApJ, 574, 740

Trump, J. R., Hall, P. B., Reichard, T. A., et al. 2006, ApJS, 165, 1

Urrutia, T., Becker, R. H., White, R. L., et al. 2009, ApJ, 698, 1095

Urry, C. M., \& Padovani, P. 1995, PASP, 107, 803

Vanden Berk, D. E., Richards, G. T., Bauer, A., et al. 2001, AJ, 122, 549

Vanden Berk, D. E., Schneider, D. P., Richards, G. T., et al. 2005, AJ, 129,2047

Vestergaard, M. 2003, ApJ, 599, 116

Vestergaard, M., \& Osmer, P. S. 2009, ApJ, 699, 800 (VO09)

Vestergaard, M., \& Peterson, B. M. 2006, ApJ, 641, 689 (VP06)

Vestergaard, M., \& Wilkes, B. J. 2001, ApJS, 134, 1

Wang, J.-G., Dong, X.-B., Wang, T.-G., et al. 2009, ApJ, 707, 1334

Weymann, R. J., Carswell, R. F., \& Smith, M. G. 1981, ARA\&A, 19, 41

Wilkes, B. J. 1984, MNRAS, 207, 73

Willmer, C. N. A., Faber, S. M., Koo, D. C., et al. 2006, ApJ, 647, 853

White, R. L., Becker, R. H., Helfand, D. J., \& Gregg, M. D. 1997, ApJ, 475,479

Wild, V., Kauffmann, G., White, S., et al. 2008, MNRAS, 388, 227

Wills, B. J., Netzer, H., Brotherton, M. S., et al. 1993, ApJ, 410, 534

Woo, J.-H., \& Urry, C. M. 2002, ApJ, 579, 530

Wright, E. L., Eisenhardt, P. R. M., Mainzer, A. K., et al. 2010, AJ, 140, 1868 